TRANSACTIONS OF THE

AMERICAN MATHEMATICAL SOCIETY

Volume 363, Number 9, September 2011, Pages 4877-4927

S 0002-9947(2011)05124-X

Article electronically published on April 14, 2011

\title{
ON THE WEIL-ÉTALE COHOMOLOGY OF NUMBER FIELDS
}

\author{
BAPTISTE MORIN
}

\begin{abstract}
We give a direct description of the category of sheaves on Lichtenbaum's Weil-étale site of a number ring. Then we apply this result to define a spectral sequence relating Weil-étale cohomology to Artin-Verdier étale cohomology. Finally we construct complexes of étale sheaves computing the expected Weil-étale cohomology.
\end{abstract}

\section{INTRODUCTION}

Stephen Lichtenbaum has conjectured in [6] the existence of a Weil-étale topology for arithmetic schemes. The associated cohomology groups with coefficients in motivic complexes of sheaves should be finitely generated and closely related to special values of zeta functions. For example, Lichtenbaum predicts that the Weil-étale cohomology groups with compact support $H_{W c}^{i}(Y ; \mathbb{Z})$ exist, are finitely generated and vanish for $i$ large, where $Y$ is a scheme of finite type over Spec $\mathbb{Z}$. The order of annulation and the special value of the zeta function $\zeta_{Y}(s)$ at $s=0$ should be given by

$$
\operatorname{ord}_{s=0} \zeta_{Y}(s)=\chi_{c}^{\prime}(Y, \mathbb{Z}) \text { and } \zeta_{Y}^{*}(0)= \pm \chi_{c}(Y, \mathbb{Z}),
$$

where $\chi_{c}(Y, \mathbb{Z})$ and $\chi_{c}^{\prime}(Y, \mathbb{Z})$ are the Euler characteristics defined in $[6$. Lichtenbaum has also defined a candidate for the Weil-étale cohomology when $Y=$ $\operatorname{Spec} \mathcal{O}_{K}$, the spectrum of a number ring. Assuming that the groups $H_{W}^{i}(\bar{Y} ; \mathbb{Z})$ vanish for $i \geq 4$, he has proven his conjecture in this case. However, Matthias Flach has shown in [4] that the groups $H_{W}^{i}(\bar{Y} ; \mathbb{Z})$ defined in [6] are in fact infinitely generated for any even integer $i \geq 4$. The aim of the present work is to study in more detail Lichtenbaum's definition and its relation to Artin-Verdier étale cohomology.

Let $K$ be a number field and let $\bar{Y}$ be the Arakelov compactification of $\operatorname{Spec} \mathcal{O}_{K}$. In the second section we define a topos $\mathfrak{F}_{L / K, S}$, said to be flask, using the Weil group $W_{L / K, S}$ associated to a finite Galois extension $L / K$ and a finite set $S$ of places of $K$ containing the archimedean ones and the places which ramify in $L$. We also define a topos $\mathfrak{F}_{W, \bar{Y}}$ using the full Weil-group $W_{K}$. The first main result of this paper shows that the topos $\mathfrak{F}_{L / K, S}$ is canonically equivalent to the category of sheaves on the Lichtenbaum Weil-étale site $T_{L / K, S}$. This gives a simple description of the

Received by the editors February 25, 2009 and, in revised form, January 6, 2010.

2000 Mathematics Subject Classification. Primary 14F20; Secondary 14G10.

Key words and phrases. Étale cohomology, Weil-étale cohomology, topos, Dedekind zeta function.

(C)2011 American Mathematical Society Reverts to public domain 28 years from publication 4877 
categories of sheaves on those Weil-étale sites. In the spirit of [5], it is sometimes easier to work directly with these flask topoi rather than with their generating sites $T_{L / K, S}$. Finally, this exhibits the somewhat unexpected behavior of these categories of sheaves.

In the third section we compute the groups $H_{W}^{i}(\bar{Y} ; \mathbb{Z}):=\underset{\lim }{\longrightarrow} H^{i}\left(\mathfrak{F}_{L / K, S}, \mathbb{Z}\right)$ and $H^{i}\left(\mathfrak{F}_{W, \bar{Y}}, \mathbb{Z}\right)$. Then we observe that the canonical map $\underset{\lim }{\longrightarrow} H^{i}\left(\mathfrak{F}_{L / K, S}, \mathbb{Z}\right) \rightarrow$ $H^{i}\left(\mathfrak{F}_{W, \bar{Y}}, \mathbb{Z}\right)$ is not an isomorphism for $i=2,3$. This points out that the current Weil-étale cohomology is not defined as the cohomology of a site (i.e. of a topos).

In the seventh section we study the relation between the flask topoi and the Artin-Verdier étale topos. This is then applied to define a spectral sequence relating Weil-étale cohomology to étale cohomology. The last section is devoted to the

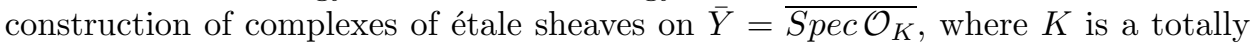
imaginary number field. The étale hypercohomology of these complexes yields the expected Weil-étale cohomology with and without compact support. This last result was suggested by a question of Matthias Flach. The existence of these complexes is a necessary condition for the existence of a Weil-étale topos (i.e. a topos whose cohomology is the conjectural Weil-étale cohomology) over the Artin-Verdier étale topos.

\section{Notation}

Let $K$ be a number field and let $\bar{K} / K$ be an algebraic closure of $K$. We denote by $Y$ the spectrum of the ring of integers $\mathcal{O}_{K}$ of $K$. Following Lichtenbaum's terminology, we call $\bar{Y}=\left(Y ; Y_{\infty}\right)$ the set of all valuations of $K$, where $Y_{\infty}$ is the set of archimedean valuations of $K$. This set $\bar{Y}$ is endowed with the Zariski topology. The trivial valuation $v_{0}$ of $K$ corresponds to the generic point of $Y$. We denote by $\bar{Y}^{0}$ the set of closed points of $\bar{Y}$ (i.e. the set of non-trivial valuations of $K$ ).

2.1. The global Weil group. Let $\bar{K} / L / K$ be a finite Galois extension of the number field $K$. Let $S$ be a finite set of places of $K$ containing the archimedean ones and the places which ramify in $L$. We denote by $I_{L}$ and $C_{L}$ the idèle group and the idèle class group of $L$, respectively. Let $U_{L, S}$ be the sub-group of $I_{L}$ consisting of those idèles which are 1 at valuations lying over $S$ and units at valuations not lying over $S$. It is well known that $U_{L, S}$ is a cohomologically trivial $G(L / K)$-module. The natural map $U_{L, S} \rightarrow C_{L}$ is injective, and the $S$-idèle class group $C_{L, S}$ is defined by $C_{L, S}=C_{L} / U_{L, S}$, as a topological group. For any $i \in \mathbb{Z}$, the map

$$
\widehat{H}^{i}\left(G(L / K), C_{L}\right) \longrightarrow \widehat{H}^{i}\left(G(L / K), C_{L, S}\right)
$$

is an isomorphism since $U_{L, S}$ is cohomologically trivial. By class field theory, there exists a canonical class in $\widehat{H}^{2}\left(G(L / K), C_{L, S}\right)$ which yields a group extension

$$
0 \rightarrow C_{L, S} \rightarrow W_{L / K, S} \rightarrow G(L / K) \rightarrow 0 .
$$

If we assume that $S$ is the set of all non-trivial valuations of $K$, then $W_{L / K, S}$ is the relative Weil group $W_{L / K}$. By [6], Lemma 3.1, the global Weil group is the projective limit

$$
W_{K}=\underset{\lim }{\longleftarrow} W_{L / K, S}
$$

over finite Galois $\bar{K} / L / K$ and finite $S$ as above. 


\subsection{Galois groups and Weil groups.}

2.2.1. For any valuation $v$ of $K$, we choose a valuation $\bar{v}$ of $\bar{K}$ lying over $v$ and we denote by $D_{v}$ the associated decomposition group and by $I_{v}$ the inertia group. We set

$$
K_{v}^{h}:=\bar{K}^{D_{v}}, K_{v}^{s h}:=\bar{K}^{I_{v}} \text { and } G_{k(v)}:=\operatorname{Gal}\left(K_{v}^{s h} / K_{v}^{h}\right)=D_{v} / I_{v}
$$

If $v \in Y$, then $k(v)$ is the residue field of the scheme $Y$ at $v$. For any archimedean valuation $v$, the Galois group $G_{k(v)}=\{1\}$ is trivial since $D_{v}=I_{v}$. Note that for the trivial valuation $v=v_{0}$, one has $D_{v_{0}}=G_{K}$ and $I_{v_{0}}=\{1\}$, hence $G_{k\left(v_{0}\right)}=G_{K}$.

Let $K_{v}$ be the completion of $K$ with respect to the valuation $v$. Thus for $v=v_{0}$ the trivial valuation, $K_{v_{0}}$ is just $K$. The choice of the valuation $\bar{v}$ of $\bar{K}$ lying over $v$ induces an embedding

$$
\mathfrak{o}_{v}: D_{v}=G_{K_{v}} \longrightarrow G_{K} .
$$

We choose a global Weil group $\alpha_{v_{0}}: W_{K} \rightarrow G_{K}$. For any non-trivial valuation $v$, we choose a local Weil group $\alpha_{K_{v}}: W_{K_{v}} \rightarrow G_{K_{v}}$ and a Weil map $\theta_{v}: W_{K_{v}} \rightarrow W_{K}$ so that the diagram

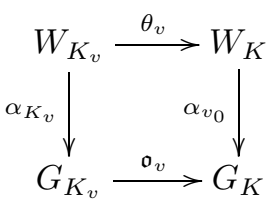

is commutative. For any valuation $v$, let $W_{k(v)}:=W_{K_{v}} / I_{v}$ be the Weil group of the residue field at $v$. Note that $W_{k(v)}$ is isomorphic to $\mathbb{Z}$ (respectively $\mathbb{R}$ ) as a topological group whenever $v$ is ultrametric (respectively archimedean). We denote by

$$
q_{v}: W_{K_{v}} \longrightarrow W_{k(v)} \text { and } \mathfrak{q}_{v}: G_{K_{v}} \longrightarrow G_{k(v)}
$$

the canonical continuous projections. One has $K_{v_{0}}=K, D_{v_{0}}=G_{K}, I_{v_{0}}=\{1\}$, and $W_{k\left(v_{0}\right)}=W_{K_{v_{0}}} / I_{v_{0}}=W_{K}$. We set $\theta_{v_{0}}=q_{v_{0}}=I d_{W_{K}}$ and $\mathfrak{o}_{v_{0}}=\mathfrak{q}_{v_{0}}=I d_{G_{K}}$.

2.2.2. Let $v$ be a non-trivial valuation of $K$ and let $W_{K_{v}} \rightarrow W_{K}$ be a Weil map. Consider the morphism

$$
W_{K_{v}} \longrightarrow W_{K} \longrightarrow W_{L / K}=W_{K} / W_{L}^{c},
$$

where $L / K$ is a finite Galois extension. Here $W_{L}^{c}$ is the closure of the commutator sub-group of $W_{L}$. The valuation $\bar{v}$ lying over $v$ defines a valuation $w$ of $L$, and the morphism $W_{K_{v}} \rightarrow W_{L / K}$ factors through $W_{K_{v}} / W_{L_{w}}^{c}=W_{L_{w} / K_{v}}$. We get the following commutative diagram:

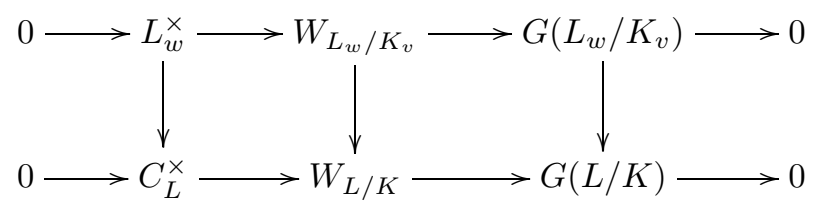

where the rows are both exact. The map $W_{L_{w} / K_{v}} \rightarrow W_{L / K}$ is injective and the image of $W_{K_{v}}$ in $W_{L / K}$ is isomorphic to $W_{L_{w} / K_{v}}$. Let $S$ be a finite set of places of $K$ containing the archimedean ones and the places which ramify in $L$. The 
group $U_{L, S}$ injects in $W_{L / K}$, and there is an isomorphism $W_{L / K, S} \simeq W_{L / K} / U_{L, S}$. Hence the image of $W_{K_{v}}$ in $W_{L / K, S}$ is isomorphic to $W_{L_{w} / K_{v}}$ for $v \in S$. For $v$ not in $S$, the image of $W_{K_{v}}$ in $W_{L / K, S}$ is isomorphic to the quotient of $W_{L_{w} / K_{v}}$ by $\mathcal{O}_{L_{w}}^{\times}$. The canonical map $W_{K_{v}} \rightarrow W_{k(v)}$ factors through $W_{L_{w} / K_{v}}$, hence through $W_{L_{w} / K_{v}} / \mathcal{O}_{L_{w}}^{\times}$. We denote by $\widetilde{W}_{K_{v}}$ the image of $W_{K_{v}}$ in $W_{L / K, S}$. For any trivial valuation $v$ of $K$, the Weil map $W_{K_{v}} \rightarrow W_{K}$ and the quotient map $W_{K_{v}} \rightarrow W_{k(v)}$ induce morphisms $\theta_{v}: \widetilde{W}_{K_{v}} \rightarrow W_{L / K, S}$ and $q_{v}: \widetilde{W}_{K_{v}} \rightarrow W_{k(v)}$, respectively.

2.3. Left exact sites. Let $\mathcal{C}$ be a category and let $\mathcal{J}$ be a Grothendieck topology on $\mathcal{C}$. Recall that a category $\mathcal{C}$ has finite projective limits if and only if $\mathcal{C}$ has a final object and fiber products.

Definition 2.1. The site $(\mathcal{C} ; \mathcal{J})$ is said to be left exact whenever $\mathcal{C}$ has finite projective limits and $\mathcal{J}$ is sub-canonical.

Note that any Grothendieck topos is equivalent to the category of sheaves of sets on a left exact site (see [5], IV, Théorème 1.2).

Definition 2.2. A family of morphisms $\left\{X_{i} \rightarrow X ; i \in I\right\}$ of the category $\mathcal{C}$ is said to be a covering family of $X$ if the sieve of $X$ generated by this family lies in $\mathcal{J}(X)$.

The covering families define a pretopology on $\mathcal{C}$ which generates the topology $\mathcal{J}$, since $\mathcal{C}$ is left exact. A morphism of left exact sites is a functor $a: \mathcal{C} \rightarrow \mathcal{C}^{\prime}$ preserving finite projective limits (i.e. $a$ is left exact), which is continuous. This means that the functor

$$
\begin{aligned}
& \widehat{\mathcal{C}^{\prime}} \longrightarrow \hat{\mathcal{C}}, \\
& \mathcal{P} \longmapsto \mathcal{P} \circ a
\end{aligned}
$$

sends sheaves to sheaves, where $\widehat{\mathcal{C}}$ is the category of presheaves on $\mathcal{C}$ (contravariant functors from $\mathcal{C}$ to the category of sets). We denote by $\widetilde{(\mathcal{C}, \mathcal{J})}$ the topos of sheaves of sets on the site $(\mathcal{C} ; \mathcal{J})$. A morphism of left exact sites $a:(\mathcal{C}, \mathcal{J}) \rightarrow\left(\mathcal{C}^{\prime}, \mathcal{J}^{\prime}\right)$ induces a morphism of topoi $\widetilde{a}=\left(\widetilde{a}^{*}, \widetilde{a}_{*}\right)$ such that the square

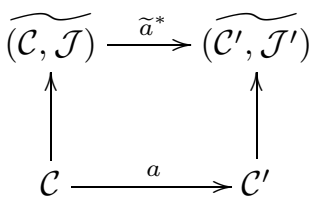

is commutative, where the vertical arrows are given by Yoneda embeddings (which are fully faithful since the topologies are sub-canonical) and $\widetilde{a}^{*}$ is the inverse image of $\widetilde{a}$. We denote by $E t_{X}$ the small étale site of a scheme $X$. The étale topos of $X$ (i.e. the category of sheaves of sets on $E t_{X}$ ) is denoted by $X_{e t}$. A morphism of schemes $u: X \rightarrow Y$ gives rise to a morphism of left exact sites

$$
\begin{aligned}
& u^{*}: E t_{Y} \longrightarrow E t_{X}, \\
& (U \rightarrow Y) \longmapsto\left(U \times_{Y} X \rightarrow X\right),
\end{aligned}
$$


hence to a morphism of topoi $\left(u^{*} ; u_{*}\right): X_{e t} \rightarrow Y_{e t}$. A diagram of topoi

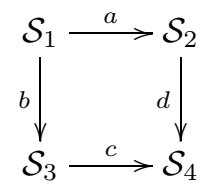

is said to be commutative if there is a canonical isomorphism of morphisms of topoi $c \circ b \simeq d \circ a$, or in other words, an isomorphism in the category Homtop $\left(\mathcal{S}_{1} ; \mathcal{S}_{4}\right)$ between the objects $c \circ b$ and $d \circ a$. Strictly speaking, such a diagram is only pseudo-commutative. In what follows, a topos is always a Grothendiek topos and a morphism is a geometric morphism.

2.4. The classifying topos of a topological group. Let $G$ be a topological group. The small classifying topos $B_{G}^{s m}$ is the category of sets on which $G$ acts continuously. If $G$ is discrete or profinite (or more generally totally disconnected), then the cohomology of the topos $B_{G}^{s m}$ is precisely the cohomology of the group $G$.

For $G$ any topological group, we denote by $B_{T o p} G$ the category of $G$-topological spaces (which are elements of a given universe) endowed with the local-section topology $\mathcal{J}_{l s}$ (see [6], section 1 ), and $B_{G}$ is the topos of sheaves of sets on this site.

Alternatively, let Top be the category of topological spaces (which are elements of a given universe) endowed with the open cover topology $\mathcal{J}_{\text {open }}$. Recall that the open cover topology is generated by the pre-topology for which a family of continuous maps $\left\{U_{i} \rightarrow U\right\}$ is a cover when it is an open cover in the usual sense. By (4], Lemma 1), one has $\mathcal{J}_{l s}=\mathcal{J}_{\text {open }}$ on the category Top. We denote by $\mathcal{T}$ the topos of sheaves of sets on the site $\left(T o p, \mathcal{J}_{\text {open }}\right)$. Since the Yoneda embedding commutes with projective limits, a topological group $G$ defines a group-object $y(G)$ of $\mathcal{T}$. The classifying topos $B_{G}$ of the topological group $G$ is the topos of $y(G)$-objects of $\mathcal{T}$. Recall that the data of an object $\mathcal{F}$ of $\mathcal{T}$ is equivalent to the following. For any topological space $X$, a sheaf $F_{X}$ on $X$ (i.e. an étalé space over $X$ ), and for any continuous map $u: X^{\prime} \rightarrow X$, a morphism $\varphi_{u}: u^{*} F_{X} \rightarrow F_{X}^{\prime}$ satisfying the natural transitivity condition for a composition $v \circ u: X^{\prime \prime} \rightarrow X^{\prime} \rightarrow X$. Moreover, $\varphi_{u}$ is an isomorphism whenever $u$ is an open immersion or more generally an étalement. This gives a description of the topoi $\mathcal{T}$ and $B_{G}$. By 4, Corollary 2, the two preceding definitions of $B_{G}$ are equivalent. In other words, $\left(B_{T o p} G ; \mathcal{J}_{l s}\right)$ is a site for the classifying topos $B_{G}$.

2.5. Cohomology of the Weil group. Let $\mathcal{E}$ be a topos. There is a unique morphism $u: \mathcal{E} \rightarrow \underline{\text { Set}}$. The left exact functor $\Gamma_{\mathcal{E}}:=u_{*}=\operatorname{Hom}_{\mathcal{E}}\left(e_{\mathcal{E}},-\right)$ is called the global sections functor. Here $e_{\mathcal{E}}$ denotes the final object of $\mathcal{E}$. For any abelian object $\mathcal{A}$ of $\mathcal{E}$, one has

$$
H^{i}(\mathcal{E}, \mathcal{A}):=R^{i}\left(\Gamma_{\mathcal{E}}\right)(\mathcal{A})
$$

For any topological group $G$ and any abelian object of $B_{G}$ (in particular a topological $G$-module), the cohomology of $G$ is defined by (see 4])

$$
H^{i}(G, \mathcal{A}):=H^{i}\left(B_{G}, \mathcal{A}\right) \text {. }
$$

The following result is due to Stephen Lichtenbaum for $i \leq 3$ and to Matthias Flach for $i>3$. Denote by $A^{\mathcal{D}}:=\operatorname{Hom}_{\text {cont }}(A, \mathbb{R} / \mathbb{Z})$ the Pontryagin dual of a locally compact abelian group $A$. The kernel of the absolute value map $C_{K} \rightarrow \mathbb{R}_{+}^{\times}$ is denoted by $C_{K}^{1}$. 
Theorem 2.3. Let $K$ be a totally imaginary number field and let $\mathbb{Z}$ be the discrete $W_{K}$-module with trivial action. Then

$$
\begin{aligned}
H^{i}\left(W_{K} ; \mathbb{Z}\right) & =\mathbb{Z} \text { for } i=0 \\
& =\left(C_{K}^{1}\right)^{\mathcal{D}} \text { for } i=2 \\
& =0 \text { for } i \text { odd },
\end{aligned}
$$

and $H^{i}\left(W_{K} ; \mathbb{Z}\right)$ is an abelian group of infinite rank, in particular non-zero, for even $i \geq 4$.

\section{The FLASK TOPOI ASSOCIATED TO A NUMBER FIELD}

3.1. Definition of the flask topoi. Let $L / K$ be an algebraic extension and let $S$ be a set of non-trivial valuations of the number field $K$ containing all the valuations of $F$ which ramify in $K$ and the archimedean ones. In what follows, either $L / K$ is a finite Galois extension and $S$ is a finite set, or $L=\bar{K} / K$ is an algebraic closure of $K$ and $S$ is the set of all non-trivial valuations of $K$. Recall that $\widetilde{W}_{K_{v}}$ denotes the image of $W_{K_{v}}$ in $W_{L / K, S}$. The chosen Weil map and the quotient map induce continuous morphisms

$$
\theta_{v}: \widetilde{W}_{K_{v}} \rightarrow W_{L / K, S} \text { and } q_{v}: \widetilde{W}_{K_{v}} \rightarrow W_{k(v)}
$$

for any valuation $v$ of $K$. For the trivial valuation $v_{0}$, the maps $\theta_{v_{0}}$ and $q_{v_{0}}$ are just $I d_{W_{L / K, S}}$.

Definition 3.1. We define a category $\mathfrak{F}_{L / K, S}$ as follows. The objects of this category are of the form $\mathcal{F}=\left(F_{v} ; f_{v}\right)_{v \in \bar{Y}}$, where $F_{v}$ is an object of $B_{W_{k(v)}}$ for $v \neq v_{0}$ (respectively of $B_{W_{L / K, S}}$ for $v=v_{0}$ ) and

$$
f_{v}: q_{v}^{*}\left(F_{v}\right) \longrightarrow \theta_{v}^{*}\left(F_{v_{0}}\right)
$$

is a morphism of $B_{\widetilde{W}_{K_{v}}}$ so that $f_{v_{0}}=I d_{F_{v_{0}}}$. A morphism $\phi$ from $\mathcal{F}=\left(F_{v} ; f_{v}\right)_{v \in \bar{Y}}$ to $\mathcal{F}^{\prime}=\left(F_{v}^{\prime} ; f_{v}^{\prime}\right)_{v \in \bar{Y}}$ is a family of morphisms $\phi_{v}: F_{v} \rightarrow F_{v}^{\prime} \in F l\left(B_{W_{k(v)}}\right)$ (and $\left.\phi_{v_{0}} \in F l\left(B_{W_{L / K, S}}\right)\right)$ so that

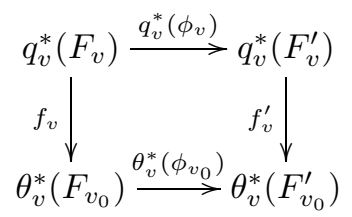

is a commutative diagram of $B_{\widetilde{W}_{K}}$. In what follows, $F_{v}$ (respectively $\phi_{v}$ ) is called the $v$-component of the object $\mathcal{F}$ (respectively of the morphism $\phi$ ).

For $L=\bar{K}$ and $S$ the set of all non-trivial valuations of $K$, one has $W_{L / K, S}=$ $W_{K}, \widetilde{W}_{K_{v}}=W_{K_{v}}$, and we set

$$
\mathfrak{F}_{L / K, S}=\mathfrak{F}_{W ; \bar{Y}} .
$$

The aim of this section is to prove that the category $\mathfrak{F}_{L / K, S}$ is a Grothendieck topos.

Proposition 3.2. Arbitrary inductive and finite projective limits exist in $\mathfrak{F}_{L / K, S}$ and are calculated componentwise. 
Proof. In order to simplify the notation we assume here that $\mathfrak{F}_{L / K, S}=\mathfrak{F}_{W ; \bar{Y}}$. Let $I$ be a small category and let $G: I \rightarrow \mathfrak{F}_{W ; \bar{Y}}$ be an arbitrary functor. For any valuation $v$ of $K$, one has a canonical functor

$$
\begin{aligned}
i_{v}^{*}: \mathfrak{F}_{W ; \bar{Y}} & \longrightarrow B_{W_{k(v)}}, \\
\mathcal{F} & \longmapsto F_{v} .
\end{aligned}
$$

For any valuation $v$, we set

$$
G_{v}:=i_{v}^{*} \circ G: I \rightarrow B_{W_{k(v)}} .
$$

The inductive limit

$$
L_{v}:=\lim _{\longrightarrow} G_{v}
$$

exists in the topos $B_{W_{k(v)}}$. A map $i \rightarrow j$ of the category $I$ induces a map $G(i) \rightarrow$ $G(j)$ of the category $\mathfrak{F}_{W ; \bar{Y}}$. Hence for any valuation $v$, one has a commutative diagram of $B_{W_{K_{v}}}$ :

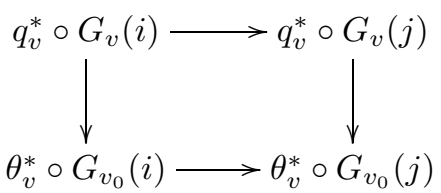

By the universal property of inductive limits, one has an induced morphism

$$
\lim _{\longrightarrow} q_{v}^{*} \circ G_{v} \longrightarrow \lim _{I} \theta_{v}^{*} \circ G_{v_{0}},
$$

where the limits are calculated in the topos $B_{W_{K_{v}}}$. We get a map

$l_{v}: q_{v}^{*}\left(L_{v}\right)=q_{v}^{*}\left(\lim _{I} G_{v}\right)=\lim _{I} q_{v}^{*} \circ G_{v} \longrightarrow \lim _{I} \theta_{v}^{*} \circ G_{v_{0}}=\theta_{v}^{*}\left(\lim _{I} G_{v_{0}}\right)=\theta_{v}^{*}\left(L_{v_{0}}\right)$, since $q_{v}^{*}$ and $\theta_{v}^{*}$ commute with arbitrary inductive limits. This yields an object

$$
\lim _{I} G=\mathcal{L}:=\left(L_{v} ; l_{v}\right)_{v \in \bar{Y}}
$$

of $\mathfrak{F}_{W ; \bar{Y}}$. Now, one has to check that $\mathcal{L}$ is the inductive limit of the functor $G$. For any object $\mathcal{X}$ of $\mathfrak{F}_{W ; \bar{Y}}$, denote by $k_{\mathcal{X}}: I \rightarrow \mathfrak{F}_{W ; \bar{Y}}$ the constant functor associated to $\mathcal{X}$. By construction, there is a natural transformation

$$
a: G \longrightarrow k_{\mathcal{L}}
$$

such that any other natural transformation

$$
b: G \longrightarrow k_{\mathcal{X}}
$$

factors through $a$. Indeed, the $v$-component of $\mathcal{L}$ is defined as the inductive limit of $G_{v}$ in $B_{W_{k(v)}}$ and the morphism $l_{v}$ is defined as the limit of the corresponding system of compatible maps of $B_{W_{K_{v}}}$. The proof for finite projective limits is identical.

Proposition 3.3. The category $\mathfrak{F}_{L / K, S}$ is a topos.

Proof. Again, we assume that $\mathfrak{F}_{L / K, S}=\mathfrak{F}_{W: \bar{Y}}$ (i.e. $L$ is an algebraic closure of $K$ and $S$ is the set of non-trivial valuations of $K$ ). To see that it is a topos, we use Giraud's criterion (see [5], IV, Théorème 1.2). Axioms (G1), (G2) and (G3) follow from Proposition 3.2 and the fact that $q_{v}^{*}$ and $\theta_{v}^{*}$ commute with finite projective limits and arbitrary inductive limits.

(G1) The category $\mathfrak{F}_{W ; \bar{Y}}$ has finite projective limits.

More explicitly, $\mathfrak{F}_{W ; \bar{Y}}$ has a final object $\left(e_{W_{k(v)}} ; f_{v}\right)_{v \in \bar{Y}}$. Here $e_{W_{k(v)}}$ is the final 
object of $B_{W_{k(v)}}$ and $f_{v}$ is the unique map from the final object of $B_{W_{K_{v}}}$ to itself. Let $\phi: \mathcal{F} \rightarrow \mathcal{X}$ and $\phi^{\prime}: \mathcal{F}^{\prime} \rightarrow \mathcal{X}$ be two maps of $\mathfrak{F}_{W: \bar{Y}}$ with the same target $\mathcal{X}=\left(X_{v} ; \xi_{v}\right)$. The fiber product $\mathcal{F} \times \mathcal{X} \mathcal{F}^{\prime}$ is defined as the object $\left(F_{v} \times X_{v} F_{v}^{\prime} ; f_{v} \times_{\xi_{v}}\right.$ $\left.f_{v}^{\prime}\right)_{v \in \bar{Y}}$, where the fiber products are calculated in the categories $B_{W_{k(v)}}$ and $B_{W_{K_{v}}}$, respectively.

(G2) All (set-indexed) sums exist in $\mathfrak{F}_{W ; \bar{Y}}$, and are disjoint and stable.

The initial object of $\mathfrak{F}_{W ; \bar{Y}}$ is $\left(\emptyset_{W_{k(v)}} ; f_{v}^{\prime}\right)_{v \in \bar{Y}}$, where $\emptyset_{W_{k(v)}}$ is the initial object of $B_{W_{k(v)}}$ and $f_{v}^{\prime}: \emptyset_{W_{K_{v}}} \rightarrow \emptyset_{W_{K_{v}}}$ is the trivial map. Moreover, fiber products are computed componentwise in $\mathfrak{F}_{W ; \bar{Y}}$, and an isomorphism $\phi$ from $\mathcal{F}=\left(F_{v} ; f_{v}\right)_{v \in \bar{Y}}$ to $\mathcal{F}^{\prime}=\left(F_{v}^{\prime} ; f_{v}^{\prime}\right)_{v \in \bar{Y}}$ is a family of compatible isomorphisms $\phi_{v}: F_{v} \rightarrow F_{v}^{\prime} \in$ $F l\left(B_{W_{k(v)}}\right)$. Then one easily sees that (G2) is satisfied by $\mathfrak{F}_{W ; \bar{Y}}$ since it is satisfied by $B_{W_{k(v)}}$ for any valuation $v$.

(G3) The equivalence relations are effective and universal.

Again this follows from the fact that arbitrary inductive limits exist and are computed componentwise in $\mathfrak{F}_{W ; \bar{Y}}$.

(G4) The category $\mathfrak{F}_{W ; \bar{Y}}$ has a small set of generators.

This axiom, however, requires some argument. Choose a small set $\left\{X_{v ; i} ; i \in I_{v}\right\}$ of generators of $B_{W_{k(v)}}$, for any valuation $v$. Recall that the morphism of topological groups $\theta_{v}: W_{K_{v}} \rightarrow W_{K}$ induces the sequence of three adjoint functors

$$
\theta_{v !} ; \theta_{v}^{*} ; \theta_{v *}
$$

between $B_{W_{K}}$ and $B_{W_{K}}$, since $\theta_{v}^{*}$ commutes with arbitrary projective and inductive limits (see [5], IV.4.5.1). The functors $\theta_{v}^{*}$ and $\theta_{v *}$ are respectively the inverse image and the direct image of the (essential) morphism $B_{\theta_{v}}: B_{W_{K_{v}}} \rightarrow B_{W_{K}}$. Denote by $y: T o p \rightarrow \mathcal{T}$ the Yoneda embedding. The functor $\theta_{v}$ ! is defined by

$$
\begin{aligned}
\theta_{v !}: B_{W_{K_{v}}} & \longrightarrow y\left(W_{K}\right) \times^{y\left(W_{K_{v}}\right)} F:=\left(y\left(W_{K}\right) \times F\right) / y\left(W_{K_{v}}\right), \\
F & \longmapsto y
\end{aligned}
$$

where $y\left(W_{K_{v}}\right)$ acts on the left on $F$ and by right-translations on $y\left(W_{K}\right)$.

Let $v \neq v_{0}$ be a non-trivial valuation and let $i \in I_{v}$. We define an object $\mathcal{X}_{v ; i}$ of $\mathfrak{F}_{W ; \bar{Y}}$ as follows:

$$
\mathcal{X}_{v ; i}=\left(\theta_{v !}\left(q_{v}^{*}\left(X_{v ; i}\right)\right) ; \quad X_{v ; i} ; \quad\left(\emptyset_{B_{W_{k}(w)}}\right)_{w \neq v_{0} ; v} ; \quad\left(\xi_{w}\right)_{w \in \bar{Y}}\right) .
$$

Here the map

$$
\xi_{v}: q_{v}^{*}\left(X_{v ; i}\right) \longrightarrow \theta_{v}^{*} \circ \theta_{v !}\left(q_{v}^{*}\left(X_{v ; i}\right)\right)
$$

is given by adjunction, and $\xi_{w}$ is the trivial map for any $w \neq v, v_{0}$. For the trivial valuation $v_{0}$ and for any $i \in I_{v_{0}}$, we set

$$
\mathcal{X}_{v_{0} ; i}:=\left(X_{v_{0} ; i} ; \quad\left(\emptyset_{B_{W_{k(w)}}}\right)_{w \neq v_{0}}\right) .
$$

The family $\left\{\mathcal{X}_{v ; i} ; v \in \bar{Y} ; i \in I_{v}\right\}$ is set indexed. We claim that it is a generating family of $\mathfrak{F}_{W ; \bar{Y}}$. Let $\mathcal{F}=\left(F_{v} ; f_{v}\right)_{v}$ be an object of $\mathfrak{F}_{W ; \bar{Y}}$, let $v$ be a valuation of $K$ and let $t_{v}: X_{v ; i} \rightarrow F_{v}$ be a morphism in $B_{W_{k(v)}}$. One needs to show that there exists a canonical morphism

$$
t: \mathcal{X}_{v ; i} \longrightarrow \mathcal{F}
$$


so that the $v$-component of $t$ is $t_{v}$. It is obvious for the trivial valuation $v=v_{0}$. Let $v \neq v_{0}$ be a non-trivial valuation. Consider the morphism

$$
f_{v} \circ q_{v}^{*}\left(t_{v}\right): q_{v}^{*}\left(X_{v ; i}\right) \longrightarrow q_{v}^{*}\left(F_{v}\right) \longrightarrow \theta_{v}^{*}\left(F_{v_{0}}\right) .
$$

By adjunction, there is an identification

$$
\operatorname{Hom}_{B_{W_{K}}}\left(\theta_{v !}\left(q_{v}^{*}\left(X_{v ; i}\right)\right) ; F_{v_{0}}\right)=\operatorname{Hom}_{B_{W_{K}}}\left(q_{v}^{*}\left(X_{v ; i}\right) ; \theta_{v}^{*}\left(F_{v_{0}}\right)\right) .
$$

Hence there exists a unique morphism

$$
t_{0}: \theta_{v !}\left(q_{v}^{*}\left(X_{v ; i}\right)\right) \longrightarrow F_{v_{0}}
$$

of $B_{W_{K}}$ corresponding to $f_{v} \circ q_{v}^{*}\left(t_{v}\right)$ via (1) so that the diagram

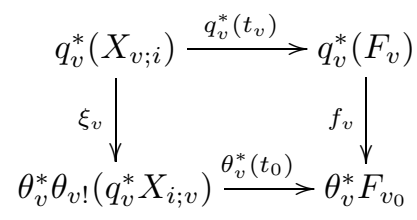

is commutative. We get a morphism $t: \mathcal{X}_{v ; i} \rightarrow \mathcal{F}$ of the category $\mathfrak{F}_{W ; \bar{Y}}$.

Now, consider two parallel arrows $\phi, \varphi: \mathcal{F} \rightarrow \mathcal{E}$ so that, for any arrow $t: \mathcal{X}_{v ; i} \rightarrow$ $\mathcal{F}$, one has $\phi \circ t=\varphi \circ t$. The family $\left\{X_{v ; i} ; i \in I_{v}\right\}$ is a family of generators of $B_{W_{k(v)}}$, and each morphism $t_{v}: X_{v ; i} \rightarrow F_{v}$ induces a morphism $t: \mathcal{X}_{v ; i} \rightarrow \mathcal{F}$. It follows that

$$
\phi_{v}=\varphi_{v} \in F l\left(B_{W_{k(v)}}\right),
$$

for any $v \in \bar{Y}$. By definition of the morphisms in the category $\mathfrak{F}_{W ; \bar{Y}}$, the functor

$$
\left(i_{v}^{*}\right)_{v \in \bar{Y}}: \mathfrak{F}_{W ; \bar{Y}} \longrightarrow \coprod_{v \in \bar{Y}} B_{W_{k(v)}}
$$

is faithful. It follows that $\phi=\varphi$. This shows that the family $\left\{\mathcal{X}_{v ; i} ; v \in \bar{Y} ; i \in\right.$ $\left.I_{v}\right\}$ is a small collection of generators of $\mathfrak{F}_{W ; \bar{Y}}$. Therefore the category $\mathfrak{F}_{W ; \bar{Y}}$ is a topos.

A topos is said to be compact if any cover of the final object by sub-objects has a finite sub-cover. A first consequence of this artificial construction is the fact that this property is not satisfied by these flask topoi, as it is shown below. As a consequence, the global sections functor (and a fortiori cohomology) does not commute with filtered inductive limits (not even with direct sums).

Proposition 3.4. The topos $\mathfrak{F}_{L / K, S}$ is not compact.

Proof. For any non-trivial valuation $v$ of $K$, let $E_{v}$ be the object of $\mathfrak{F}_{L / K, S}$ defined as follows. The $w$-component of $E_{v}$ is the initial object $\emptyset$ of $B_{W_{k(w)}}$ for $w \neq v, v_{0}$ and the final object for $w=v, v_{0}$ (i.e. the sheaf represented by the one point space with trivial action). Let $e$ be the final object of $\mathfrak{F}_{L / K, S}$. The unique map $E_{v} \rightarrow e$ is mono, hence $E_{v}$ is a sub-object of the final object of $\mathfrak{F}_{L / K, S}$. The family $\left\{E_{v} \rightarrow e, v \neq v_{0}\right\}$ is epimorphic. It is therefore a covering family of $e$ by sub-objects. However, any finite sub-family is not a covering family. 
3.2. The morphisms associated to the valuations. A valuation $v$ of the number field $K$ can be seen as a morphism $v \rightarrow \bar{Y}$ inducing in turn a morphism of topoi.

Proposition 3.5. For any non-trivial valuation $v$, there is a closed embedding:

$$
i_{v}:=i_{L, S, v}: B_{W_{k(v)}} \longrightarrow \mathfrak{F}_{L / K, S} .
$$

Proof. For any valuation $v \neq v_{0}$, the functor

$$
\begin{aligned}
i_{v}^{*}: \mathfrak{F}_{L / K, S} & \longrightarrow B_{W_{k(v)}}, \\
\mathcal{F} & \longmapsto F_{v}
\end{aligned}
$$

commutes with arbitrary inductive limits and finite projective limits, since these limits are computed componentwise in the topos $\mathfrak{F}_{L / K, S}$. Hence $i_{v}^{*}$ is the pull-back of a morphism of topoi $i_{v}: B_{W_{k(v)}} \rightarrow \mathfrak{F}_{L / K, S}$. The same argument shows that there is a morphism $j_{L / K, S}: B_{W_{L / K, S}} \rightarrow \mathfrak{F}_{L / K, S}$. Moreover, one easily sees that the functor

$$
\begin{aligned}
i_{v *}: B_{W_{k(v)}} & \longrightarrow\left(e_{W_{K}} ; F_{v} ;\left(e_{W_{k(w)}}\right)_{w \neq v ; v_{0}}\right) \\
F_{v} & \longmapsto
\end{aligned}
$$

is right adjoint to $i_{v}^{*}$, where $e_{W_{k(w)}}$ is the final object of $B_{W_{k(w)}}$. Since the adjunction transformation $I d \rightarrow i_{v}^{*} \circ i_{v *}$ is obviously an isomorphism, the morphism $i_{v}$ is an embedding (see [5], IV, Définition 9.1.1). Consider the sub-terminal object $U:=\left(\left(e_{W_{k(w)}}\right)_{w \neq v} ; \emptyset_{W_{k(v)}}\right)$ of $\mathfrak{F}_{L / K, S}$. It defines an open sub-topos

$$
j_{v}: \mathcal{U}:=\left(\mathfrak{F}_{L / K, S}\right)_{/ U} \longrightarrow \mathfrak{F}_{L / K, S} .
$$

The image of $i_{v *}$ is exactly the strictly full sub-category of $\mathfrak{F}_{L / K, S}$ defined by the objects $X$ such that $j_{v}^{*}(X)$ is the final object of $\mathcal{U}$. Hence the image of $i_{v}$ is the closed complement of the open sub-topos $\mathcal{U}$ (see [5], IV, Proposition 9.3.4).

The following corollary follows from the fact that $i_{v *}$ is a closed embedding (see [5], IV.14).

Corollary 3.6. The functor induced by $i_{v *}$ between the categories of abelian sheaves is exact.

More precisely, the functor $i_{v *}$ (between abelian categories) has a left adjoint $i_{v}^{*}$ and a right adjoint $i_{v}^{!}$(in fact one has six adjoint functors). This last functor is defined as follows:

$$
\begin{aligned}
& i_{v}^{!}: \quad A b\left(\mathfrak{F}_{L / K, S}\right) \quad \longrightarrow \quad A b\left(B_{W_{k(v)}}\right), \\
& \left(F_{w}, f_{w}\right)_{w \in \bar{Y}} \longmapsto \operatorname{Ker}\left(f_{v}\right) \text {. }
\end{aligned}
$$

A morphism of topos $j=\left(j^{*}, j_{*}\right)$ is said to be essential if the inverse image $j^{*}$ has a left adjoint $j !$.

Proposition 3.7. There is an essential morphism $j:=j_{L / K, S}: B_{W_{L / K, S}} \longrightarrow$ $\mathfrak{F}_{L / K, S}$.

Proof. The functor

$$
\begin{aligned}
j^{*}: \mathfrak{F}_{L / K, S}, & \longrightarrow B_{W_{L / K, S}}, \\
\mathcal{F} & \longmapsto F_{v_{0}}
\end{aligned}
$$

commutes with arbitrary inductive limits and finite projective limits. Therefore $j^{*}$ has a right adjoint $j_{*}$ and thus is the pull-back of a morphism of topoi. We define

$$
\begin{aligned}
j_{!}: B_{W_{L / K, S}} & \longrightarrow \mathfrak{F}_{L / K, S}, \\
\mathcal{L} & \longmapsto\left(F_{v}, f_{v}\right)_{v \in \bar{Y}},
\end{aligned}
$$


where $F_{v_{0}}=\mathcal{L}$ and $F_{v}=\emptyset$ is the initial object of $B_{W_{k(v)}}$ for any $v \neq v_{0}$. The map $f_{v}$ is the unique map from the initial object of $B_{\widetilde{W}_{K_{v}}}$ to $\theta_{v}^{*} \mathcal{L}$. Clearly, $j_{\text {! }}$ is left adjoint to $j^{*}$.

Proposition 3.8. The direct image functor $j_{*}$ is given by

$$
\begin{aligned}
j_{*}: B_{W_{L / K, S}} & \longrightarrow \\
\mathcal{L} & \longmapsto\left(q_{v *} \theta_{v}^{*} \mathcal{L}, l_{v}\right)_{v \in \bar{Y}},
\end{aligned}
$$

where the map

$$
l_{v}: q_{v}^{*} q_{v *} \theta_{v}^{*} \mathcal{L} \longrightarrow \theta_{v}^{*} q_{v_{0} *} \theta_{v_{0}}^{*} \mathcal{L}=\theta_{v}^{*} \mathcal{L}
$$

is induced by the natural transformation $q_{v}^{*} q_{v *} \rightarrow I d$, for any valuation $v$.

Proof. One has to show that $j_{*}$ is right adjoint to $j^{*}$. Let $\mathcal{L}$ be an object of $B_{W_{L / K, S}}$ and let $\mathcal{F}=\left(F_{v} ; f_{v}\right)_{v \in \bar{Y}}$ be an object of $\mathfrak{F}_{L / K, S}$. For any map $\phi_{0}: F_{0} \rightarrow \mathcal{L}$ of $B_{W_{L / K, S}}$ and any non-trivial valuation $v$, consider the map

$$
\theta_{v}^{*}\left(\phi_{0}\right) \circ f_{v}: q_{v}^{*} F_{v} \rightarrow \theta_{v}^{*} F_{0} \rightarrow \theta_{v}^{*} \mathcal{L} .
$$

Since $q_{v}^{*}$ is left adjoint to $q_{v *}$, there exists a unique map $\phi_{v}: F_{v} \rightarrow q_{v *} \theta_{v}^{*} \mathcal{L}$ such that the diagram

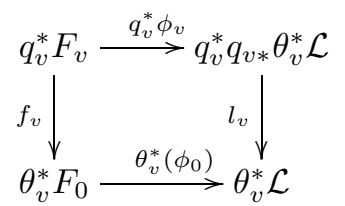

is commutative. We obtain a functorial isomorphism

$$
\operatorname{Hom}_{B_{W_{L / K}, S}}\left(j^{*} \mathcal{F}, \mathcal{L}\right) \simeq \operatorname{Hom}_{\mathfrak{F}_{L / K, S}}\left(\mathcal{F}, j_{*} \mathcal{L}\right) .
$$

Corollary 3.9. The morphism $j: B_{W_{L / K, S}} \rightarrow \mathfrak{F}_{L / K, S}$ is an embedding.

Proof. Indeed for any object $\mathcal{L}$ of $B_{W_{L / K, S}}$, the natural map $j^{*} j_{*} \mathcal{L} \rightarrow \mathcal{L}$ is just the identity of $\mathcal{L}$.

If there is no risk of ambiguity, we denote $\widetilde{W}_{k(v)}=W_{k(v)}$ for $v \neq v_{0}, \widetilde{W}_{k\left(v_{0}\right)}=$ $W_{L / K, S}$ and $j=i_{v_{0}}$.

Proposition 3.10. The family of functors

$$
\left\{i_{v}^{*}: \mathfrak{F}_{L / K, S} \rightarrow B_{\widetilde{W}_{k(v)}}, v \in \bar{Y}\right\}
$$

is conservative.

Proof. This follows immediately from the definitions.

Proposition 3.11. The family of functors

$$
\left\{i_{v}^{*}: \mathfrak{F}_{L / K, S} \longrightarrow B_{W_{k(v)}} ; v \in \bar{Y}^{0}\right\}
$$

is not conservative.

Proof. In order to simplify the notation, we assume here that $\mathfrak{F}_{L / K, S}=\mathfrak{F}_{W ; \bar{Y}}$ Let $\emptyset$ be the initial object of $\mathfrak{F}_{W: \bar{Y}}$ and let $\mathcal{G}$ be the object whose $v_{0}$-component is the final object of $B_{W_{K}}$ while its $v$-component is the initial object of $B_{W_{k(v)}}$ for any $v \neq v_{0}$. Consider the morphism $\phi: \emptyset \rightarrow \mathcal{G}$. Then $\phi$ is not an isomorphism, while $i_{v}^{*}(\phi): \emptyset_{W_{k(v)}} \rightarrow \emptyset_{W_{k(v)}}$ is an isomorphism for any closed point $v$. 
3.3. The transition maps. Let $(L / K, S)$ and $\left(L^{\prime} / K, S^{\prime}\right)$ be as above. If $L \subset L^{\prime}$ in $\bar{K}$ and $S \subset S^{\prime}$, then there is a canonical morphism

$$
p: W_{L^{\prime} / K, S^{\prime}} \longrightarrow W_{L / K, S} \text {. }
$$

Proposition 3.12. There is an induced morphism of topoi

$$
t: \mathfrak{F}_{L^{\prime} / K, S^{\prime}} \longrightarrow \mathfrak{F}_{L / K, S} .
$$

For $L^{\prime \prime} / L^{\prime} / L$ and $S \subset S^{\prime} \subset S^{\prime \prime}$, the diagram

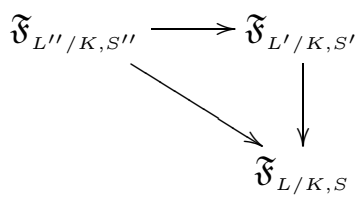

is commutative.

In the following proof, for any non-trivial valuation $v$ we denote by $W_{K_{v}, L, S}$ the image of $W_{K_{v}}$ in $W_{L / K, S}$ (this group is denoted by $\widetilde{W}_{K_{v}}$ in the rest of the paper). Let $\theta_{v, L, S}: W_{K_{v}, L, S} \rightarrow W_{L / K, S}$ and $q_{v, L, S}: W_{K_{v}, L, S} \rightarrow W_{k(v)}$ be the induced morphisms. One has a continuous map $p_{v}: W_{K_{v}, L^{\prime}, S^{\prime}} \rightarrow W_{K_{v}, L, S}$.

Proof. Let $\mathcal{F}=\left(F_{v} ; f_{v}\right)_{v \in \bar{Y}}$ be an object of $\mathfrak{F}_{L / K, S}$. Then,

$$
t^{*} \mathcal{F}=\left(p^{*} F_{v_{0}}, F_{v}, p_{v}^{*} f_{v}\right)
$$

does define an object of $\mathfrak{F}_{L^{\prime} / K, S^{\prime}}$. Indeed, $p_{v}^{*} f_{v}$ gives a map

$$
q_{v, L^{\prime}, S^{\prime}}^{*} F_{v}=p_{v}^{*} q_{v, L, S}^{*} F_{v} \longrightarrow p_{v}^{*} \theta_{v, L, S}^{*} F_{0}=\theta_{v, L^{\prime}, S^{\prime}}^{*} p^{*} F_{0},
$$

since the diagram of topological groups

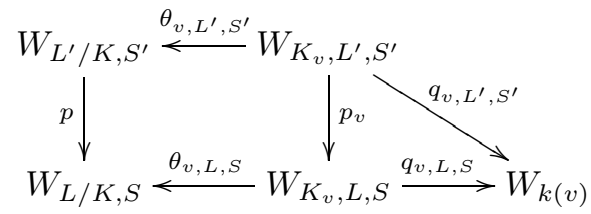

is commutative. This yields a functor

$$
t^{*}: \mathfrak{F}_{L / K, S} \longrightarrow \mathfrak{F}_{L^{\prime} / K, S^{\prime}},
$$

which commutes with finite projective limits and arbitrary inductive limits by Proposition 3.2 Hence $t^{*}$ is the pull-back of a morphism of topoi $t$. The diagram of the proposition is easily seen to be commutative, using the commutativity of the following triangles:

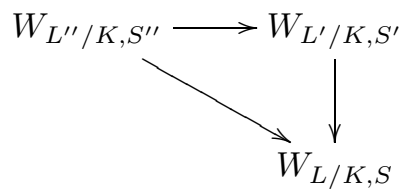

and

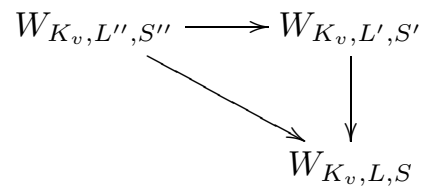

Remark 3.13. The family $\left(\mathfrak{F}_{L / K, S}\right)_{L / K, S}$ is a projective system of topoi. Indeed, consider the filtered set $I / K$ consisting of pairs $(L / K, S)$, where $L / K$ is a finite Galois sub-extension of $\bar{K} / K$ and $S$ is a finite set of places of $K$ containing the 
archimedean ones and the places ramified in $L / K$. There is an arrow $\left(L^{\prime} / K, S^{\prime}\right) \rightarrow$ $(L / K, S)$ if and only if $L \subseteq L^{\prime} \subseteq \bar{K}$ and $S \subseteq S^{\prime}$. The previous proposition shows that one has a pseudo-functor

$$
\begin{array}{cc}
\mathfrak{F}_{\bullet}: \quad I / K & \longrightarrow \frac{\text { Topos }}{\mathfrak{F}_{L / K, S}} . \\
(L / K, S) & \longmapsto \frac{1}{}
\end{array}
$$

Proposition 3.14. The diagrams
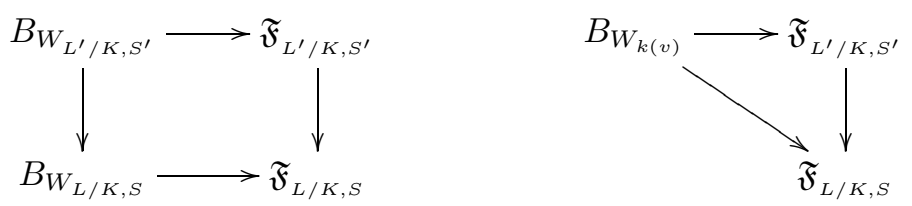

are both commutative for any non-trivial valuation $v$.

Proof. This follows immediately from the definition of $t$.

For any $v \in \bar{Y}$, there is a canonical morphism of topological groups

$$
l_{v}: W_{k(v)} \rightarrow \mathbb{R} .
$$

For the trivial valuation $v=v_{0}$, the map $l_{v_{0}}$ is defined as follows:

$$
l_{v_{0}}: W_{k\left(v_{0}\right)}=W_{K} \longrightarrow W_{K}^{a b} \simeq C_{K} \longrightarrow \mathbb{R}^{>0} \longrightarrow \mathbb{R} .
$$

The first map is the projection from $W_{K}$ to its maximal abelian Hausdorff quotient. The second map is given by the absolute value map from the idèle class group $C_{K}$ to $\mathbb{R}^{>0}$. The third map is the logarithm.

Let $\operatorname{Pic}(\bar{Y})$ be the topological group obtained by dividing the idèle class group $C_{K}$ by the unit idèles. This group is known as the Arakelov Picard group. The map $C_{K} \rightarrow \mathbb{R}$ defined as above induces a map $\operatorname{Pic}(\bar{Y}) \rightarrow \mathbb{R}$. One also has a continuous morphism $W_{k(v)} \rightarrow \operatorname{Pic}(\bar{Y})$, for any non-trivial valuation $v$. This yields the map

$$
l_{v}: W_{k(v)} \rightarrow \operatorname{Pic}(\bar{Y}) \rightarrow \mathbb{R} .
$$

Note that if $v$ is ultrametric, then $l_{v}$ sends the canonical generator of $W_{k(v)}$ to $\log (N(v)) \in \mathbb{R}$, where $N(v)=|k(v)|$ is the norm of the closed point $v$ of the scheme $Y$. Finally, the map $l_{v_{0}}$ induces a morphism $l_{L, S}: W_{L / K, S} \rightarrow \mathbb{R}$. We have an induced morphism of classifying topoi:

$$
B_{l_{L, S}}: B_{W_{L / K, S}} \longrightarrow B_{\mathbb{R}}
$$

Proposition 3.15. There is a morphism

$$
f_{L / K, S}: \mathfrak{F}_{L / K, S} \longrightarrow B_{\mathbb{R}}
$$

so that $f_{L / K, S} \circ i_{v}$ is isomorphic to $B_{l_{v}}$ for any closed point $v$ of $\bar{Y}$.

Proof. In order to simplify the notation, we assume that $\mathfrak{F}_{L / K, S}=\mathfrak{F}_{W ; \bar{Y}}$. The functor $\left(B_{l_{v}}^{*}\right)_{v}: B_{\mathbb{R}} \rightarrow \coprod_{v} B_{W_{k(v)}}$ factors through $\mathfrak{F}_{W ; \bar{Y}}$. Indeed, for any object $\mathcal{F}$ of $B_{\mathbb{R}}$, define

$$
f^{*}(\mathcal{F}):=\left(B_{l_{v}}^{*}(\mathcal{F}) ; I d_{B_{L_{v}}^{*}(\mathcal{F})}\right)_{v \in \bar{Y}} .
$$

Here

$$
I d_{B_{L_{v}}^{*}(\mathcal{F})}: q_{v}^{*} B_{l_{v}}^{*}(\mathcal{F})=B_{L_{v}}^{*}(\mathcal{F}) \longrightarrow B_{L_{v}}^{*}(\mathcal{F})=\theta_{v}^{*} B_{l_{v_{0}}}^{*}(\mathcal{F})
$$


is the identity of the object $B_{L_{v}}^{*}(\mathcal{F})$ of the category $B_{W_{K_{v}}}$, where $L_{v}: W_{K_{v}} \rightarrow \mathbb{R}$ is the canonical morphism. This is well defined since the square

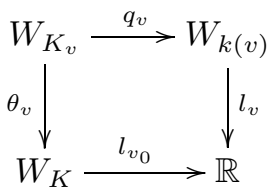

is commutative and $L_{v}:=l_{v_{0}} \circ \theta_{v}=l_{v} \circ q_{v}$. This yields a functor

$$
f^{*}: B_{\mathbb{R}} \longrightarrow \mathfrak{F}_{W ; \bar{Y}},
$$

which commutes with finite projective limits and arbitrary inductive limits by Proposition 3.2. Hence $f^{*}$ is the pull-back of a morphism of topoi $f$ such that there is an isomorphism of functors $i_{v}^{*} \circ f^{*} \simeq B_{l_{v}}^{*}$. For finite $L / K$ and finite $S$, the same construction is valid by replacing $W_{K}$ with $W_{L / K, S}$ and $W_{K_{v}}$ with $\widetilde{W}_{K_{v}}$.

Proposition 3.16. The following diagram is commutative for any $\bar{K} / L^{\prime} / L / K$ and $S \subset S^{\prime}:$

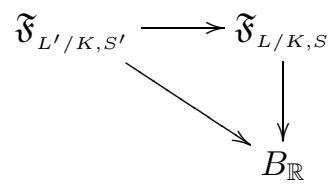

The proof is left to the reader.

Proposition 3.17. Let $\mathcal{L}$ be an object of $\mathcal{T}$ with trivial $y\left(W_{L / K, S}\right)$-action. We also denote by $\mathcal{L}$ the object of $B_{\mathbb{R}}$ defined by $\mathcal{L}$ with trivial $y(\mathbb{R})$-action. There is an isomorphism

$$
j_{L / K, S}{ }^{*} \mathcal{L} \simeq f_{L / K, S}^{*} \mathcal{L} .
$$

Proof. On the one hand, one has $f_{L / K, S}^{*} \mathcal{L}=\left(F_{v}, f_{v}\right)_{v \in \bar{Y}}$, where $F_{v}$ is defined by the trivial action on $\mathcal{L}$, for any valuation $v$. The map $f_{v}$ is given by the identity of $\mathcal{L}$.

On the other hand, one has $j_{L / K, S^{*}} \mathcal{L}=\left(q_{v *} \theta_{v}^{*} \mathcal{L}, l_{v}\right)$. Let $v$ be a non-trivial valuation of $K$. The object $\theta_{v}^{*} \mathcal{L}$ is $\mathcal{L}$ with trivial $y\left(\widetilde{W}_{K_{v}}\right)$-action. The map $\mathcal{L} \rightarrow$ $q_{v *} q_{v}^{*} \mathcal{L}$ given by adjunction is an isomorphism. It follows that $q_{v *} \mathcal{L}$ is $\mathcal{L}$ with trivial $y\left(W_{k(v)}\right)$-action.

Remark 3.18. In particular the following assertions hold. Let $\mathbb{Z}$ be the constant object of $\mathcal{T}$. Then $j_{L / K, S} * \mathbb{Z}$ is the constant object of $\mathfrak{F}_{L / K, S}$ associated to $\mathbb{Z}$. Let $\widetilde{\mathbb{R}}$ be the object of $\mathcal{T}$ represented by the topological group $\mathbb{R}$. Then $j_{L / K, S} \widetilde{\mathbb{R}}$ is the constant object of $\mathfrak{F}_{L / K, S}$ (over $\mathcal{T}$ ) associated to $\widetilde{\mathbb{R}}$. In other words, the $v$-component of $j_{L / K, S} * \widetilde{\mathbb{R}}$ is $\widetilde{\mathbb{R}}$ for any valuation $v$ with $I d_{\widetilde{\mathbb{R}}}$ as specialization maps.

\section{Cohomology}

In order to use the results of [4, we assume in this section that $K$ is a totally imaginary number field. We compute the cohomology of the total flask topos and the Lichtenbaum Weil-étale cohomology of any open subset of $\bar{Y}$. The Lichtenbaum Weil-étale cohomology is defined as a direct limit and requires some precautions to be computed rigorously. 
4.1. Preliminaries. Recall that $\theta_{v_{0}}=q_{v_{0}}=I d_{W_{L / K, S}}$. In particular the direct image of the induced morphism of topoi $q_{v_{0} *}: B_{W_{L / K, S}} \rightarrow B_{W_{L / K, S}}$ is the identity functor. Hence $R^{n}\left(q_{v_{0} *}\right)=0$ for $n \geq 1$.

Proposition 4.1. Let $\mathcal{A}$ be an abelian object of $B_{W_{L / K, S}}$. For any $n \geq 0$, one has

$$
R^{n}\left(j_{L, S *}\right)(\mathcal{A})=\left(R^{n}\left(q_{v *}\right) \theta_{v}^{*} \mathcal{A}, t_{v}\right) .
$$

Here the map $t_{v}$ is the trivial map

$$
t_{v}: q_{v}^{*} R^{n}\left(q_{v *}\right) \theta_{v}^{*} \mathcal{A} \longrightarrow \theta_{v}^{*} R^{n}\left(q_{v_{0}}\right) \theta_{v_{0}}^{*} \mathcal{A}=0,
$$

for $n \geq 1$.

Proof. In this proof, we denote the morphism $j_{L, S}$ simply by $j$. For any $n \geq 1$, one has

$$
j^{*} R^{n}\left(j_{*}\right) \mathcal{A}=R^{n}\left(j^{*} j_{*}\right) \mathcal{A}=R^{n}(I d) \mathcal{A}=0 .
$$

Indeed, the functor $j^{*}$ is exact and $j_{*}$ preserves injective objects. Hence the spectral sequence

$$
R^{p}\left(j^{*}\right) R^{q}\left(j_{*}\right)(\mathcal{A}) \Rightarrow R^{p+q}\left(j^{*} j_{*}\right)(\mathcal{A})
$$

degenerates and (2) follows. Let $v$ be a non-trivial valuation. One has $i_{v}^{*} j_{*} \mathcal{A}=$ $q_{v *} \theta_{v}^{*} \mathcal{A}$; hence

$$
i_{v}^{*} R^{n}\left(j_{*}\right) \mathcal{A}=R^{n}\left(i_{v}^{*} j_{*}\right) \mathcal{A}=R^{n}\left(q_{v *} \theta_{v}^{*}\right) \mathcal{A},
$$

since $i_{v}^{*}$ is exact. By ([5], IV.5.8), $\theta_{v}$ is a localization morphism, since $y\left(\widetilde{W}_{K_{v}}\right)$ is a sub-group of $y\left(W_{L / K, S}\right)$ in $\mathcal{T}$. It follows that $\theta_{v}^{*}$ is exact and preserves injective objects. The associated spectral sequence yields

$$
R^{n}\left(q_{v *} \theta_{v}^{*}\right) \mathcal{A}=R^{n}\left(q_{v *}\right) \theta_{v}^{*} \mathcal{A} .
$$

The proposition follows.

Recall that one has a projective system of topoi (see Remark 3.13)

$$
\mathfrak{F} \bullet: ~_{I / K} \longrightarrow \frac{\text { Topos }}{\mathfrak{F}_{L / K, S}} .
$$

The total topos Top $\left(\mathfrak{F}_{\bullet}\right)$ is defined as follows (see [5], VI.7.4). An object of Top ( $\left.\mathfrak{F}_{\bullet}\right)$ is given by a family of objects $\mathcal{F}_{L / K, S}$ of $\mathfrak{F}_{L / K, S}$ for $(L / K, S) \in I / K$, endowed with a system of compatible maps $f_{t}: t^{*} \mathcal{F}_{L / K, S} \rightarrow \mathcal{F}_{L^{\prime} / K, S^{\prime}}$. Here $t: \mathfrak{F}_{L^{\prime} / K, S^{\prime}} \rightarrow \mathfrak{F}_{L / K, S}$ is the morphism of topoi induced by the map $\left(L^{\prime} / K, S^{\prime}\right) \rightarrow(L / K, S)$ in $I / K$. The maps $f_{t}$ are compatible in the following sense. For any pair of transition maps

$$
t \circ t^{\prime}: \mathfrak{F}_{L^{\prime \prime} / K, S^{\prime \prime}} \longrightarrow \mathfrak{F}_{L^{\prime} / K, S^{\prime}} \longrightarrow \mathfrak{F}_{L / K, S},
$$

one has

$$
f_{t^{\prime}} \circ t^{\prime *}\left(f_{t}\right)=f_{t \circ t^{\prime}}: t^{\prime *} t^{*} \mathcal{F}_{L / K, S} \rightarrow t^{\prime *} \mathcal{F}_{L^{\prime} / K, S^{\prime}} \rightarrow \mathcal{F}_{L^{\prime \prime} / K, S^{\prime \prime}} .
$$

The arrows of the category Top $\left(\mathfrak{F}_{\bullet}\right)$ are the obvious ones.

Example 4.2. Denote by $t_{L, S}: \mathfrak{F}_{W, \bar{Y}} \longrightarrow \mathfrak{F}_{L / K, S}$ the canonical morphism. Let $\mathcal{F}$ be an object of $\mathfrak{F}_{W, \bar{Y}}$ and let $\mathcal{F}_{L, S}:=t_{L, S, *} \mathcal{F}$. One has $\mathcal{F}_{L, S}=t_{*} \mathcal{F}_{L^{\prime}, S^{\prime}}$, where $t: \mathfrak{F}_{L^{\prime} / K, S^{\prime}} \rightarrow \mathfrak{F}_{L / K, S}$ is the transition map. By adjunction, we have a map

$$
f_{t}: t^{*} \mathcal{F}_{L, S}=t^{*} t_{*} \mathcal{F}_{L^{\prime}, S^{\prime}} \longrightarrow \mathcal{F}_{L^{\prime}, S^{\prime}} .
$$

These maps $f_{t}$ are compatible; hence $\left(\mathcal{F}_{L, S}, f_{t}\right)$ is an object of $T o p\left(\mathfrak{F}_{\bullet}\right)$. 
Consider a discrete abelian group $A$. For any transition map $t: \mathfrak{F}_{L^{\prime} / K, S^{\prime}} \rightarrow$ $\mathfrak{F}_{L / K, S}$ we have $t^{*} A=A$, since the morphism from $\mathfrak{F}_{L^{\prime} / K, S^{\prime}}$ to the final topos $\underline{\text { Set }}$ is unique. Therefore any discrete abelian group defines an abelian object of Top $\left(\mathfrak{F}_{\bullet}\right)$ (which is the constant abelian object of the topos Top $\left(\mathfrak{F}_{\bullet}\right)$ associated to $A$ ).

More generally, Proposition 3.16 provides us with a morphism $\operatorname{Top}\left(\mathfrak{F}_{\bullet}\right) \rightarrow B_{\mathbb{R}}$ whose inverse image functor is given by

$$
\begin{aligned}
& B_{\mathbb{R}} \longrightarrow T o p\left(\mathfrak{F}_{\bullet}\right), \\
& \mathcal{F} \longmapsto\left(f_{L / K, S}^{*} \mathcal{F}\right)_{L / K, S} .
\end{aligned}
$$

Therefore, any abelian object $\mathcal{A}$ of $B_{\mathbb{R}}$ defines an abelian object $\left(f_{L / K, S}^{*} \mathcal{A}\right)$ of $\operatorname{Top}\left(\mathfrak{F}_{\bullet}\right)$. We denote this abelian object of $\operatorname{Top}\left(\mathfrak{F}_{\bullet}\right)$ by $\mathcal{A}$ also.

Proposition 4.3. Let $(L / K, S)$ be an element of $I / K$. There is an essential morphism

$$
\left(\delta_{!}, \delta^{*}, \delta_{*}\right): \mathfrak{F}_{L / K, S} \longrightarrow \operatorname{Top}\left(\mathfrak{F}_{\bullet}\right),
$$

whose inverse image is the functor

$$
\begin{aligned}
& \delta^{*}: \quad \operatorname{Top}\left(\mathfrak{F}_{\bullet}\right) \quad \longrightarrow \mathfrak{F}_{L / K, S}, \\
& \left(\mathcal{F}_{L, S}\right)_{(L, S) \in I / K} \longmapsto \mathcal{F}_{L, S} .
\end{aligned}
$$

Furthermore, $\delta_{!}$is exact; hence $\delta^{*}$ preserves injective objects.

Proof. This is ([5], VI, Lemme 7.4.12).

Definition 4.4. Let $\mathcal{A}=\left(\mathcal{A}_{L / K, S}, f_{t}\right)_{L / K, S}$ be an abelian object of the total topos Top (F্•). Lichtenbaum's Weil-étale cohomology with coefficients in $\mathcal{A}$ is defined as the inductive limit

$$
\underline{H}^{i}\left(\mathfrak{F}_{L / K, S}, \mathcal{A}\right):=\lim _{L / K, S} H^{i}\left(\mathfrak{F}_{L / K, S}, \mathcal{A}_{L / K, S}\right),
$$

where $(L / K, S)$ runs over the set of finite Galois extensions and finite $S$.

We denote by $p_{L, S}: W_{K} \rightarrow W_{L / K, S}$ the canonical map and also by $p_{L, S}:$ $B_{W_{K}} \rightarrow B_{W_{L / K, S}}$ the induced morphism of classifying topoi.

Lemma 4.5. Let $\mathcal{A}$ be an abelian object of $B_{W_{K}}$ and define $\mathcal{A}_{L, S}:=p_{L, S, *} \mathcal{A}$. The family $\left(R^{q}\left(j_{L, S *}\right) \mathcal{A}_{L, S}\right)$ defines an abelian object $\underline{R}^{q} j_{*} \mathcal{A}$ of the total topos Top $\left(\mathfrak{F}_{\bullet}\right)$.

Proof. The diagram of the topoi

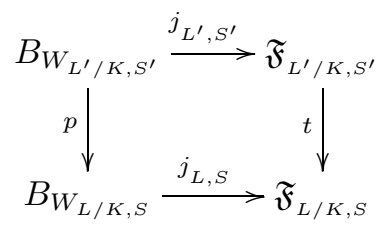

is commutative. In other words, there is an isomorphism $j_{L, S, *} p_{*} \simeq t_{*} j_{L^{\prime}, S^{\prime}, *}$. We get a transformation

$$
t^{*} j_{L, S, *} p_{*} \simeq t^{*} t_{*} j_{L^{\prime}, S^{\prime}, *} \longrightarrow j_{L^{\prime}, S^{\prime}, *},
$$

which is given by adjunction $t^{*} t_{*} \rightarrow I d$. There is an induced transformation

$$
t^{*} R^{n}\left(j_{L, S, *} p_{*}\right)=R^{n}\left(t^{*} j_{L, S, *} p_{*}\right) \longrightarrow R^{n}\left(j_{L^{\prime}, S^{\prime}, *}\right)
$$

where the identity comes from the exactness of $t^{*}$. Now, the Leray spectral sequence

$$
R^{i}\left(j_{L, S, *}\right) R^{j}\left(p_{*}\right) \Rightarrow R^{i+j}\left(j_{L, S, *} p_{*}\right)
$$


yields a natural transformation

$$
R^{n}\left(j_{L, S, *}\right) p_{*} \longrightarrow R^{n}\left(j_{L, S, *} p_{*}\right) .
$$

Composing (3) and (4), we get a transformation

$$
t^{*} R^{n}\left(j_{L, S, *}\right) p_{*} \longrightarrow R^{n}\left(j_{L^{\prime}, S^{\prime}, *}\right) .
$$

On the other hand one has $p_{*} \mathcal{A}_{L^{\prime}, S^{\prime}}=\mathcal{A}_{L, S}$, since the diagram

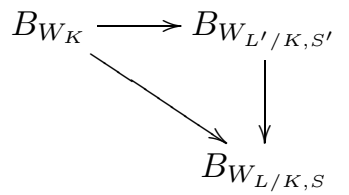

is commutative. Hence there is a canonical map

$$
f_{t}: t^{*} R^{n}\left(j_{L, S, *}\right) \mathcal{A}_{L, S}=t^{*} R^{n}\left(j_{L, S, *}\right) p_{*} \mathcal{A}_{L^{\prime}, S^{\prime}} \longrightarrow R^{n}\left(j_{L^{\prime}, S^{\prime}, *}\right) \mathcal{A}_{L^{\prime}, S^{\prime}}
$$

This yields a system of compatible maps; hence an abelian object of Top (F•).

Consider for example a topological $W_{K}$-module $A$. Let $\mathcal{A}$ be the abelian object of $B_{W_{K}}$ represented by $A$. Then $\mathcal{A}_{L, S}$ is the object of $B_{W_{L / K, S}}$ represented by $A^{N_{L, S}}$, where $N_{L, S}$ is the kernel of the map $p_{L, S}: W_{K} \rightarrow W_{L / K, S}$. The map $f_{t}$ is induced by the inclusion $A^{N_{L, S}} \hookrightarrow A^{N_{L^{\prime}, S^{\prime}}}$.

Proposition 4.6. Let $\mathcal{A}$ be an abelian object of $B_{W_{K}}$. There is a spectral sequence

$$
\stackrel{H}{\rightarrow}^{p}\left(\mathfrak{F}_{L / K, S}, \underline{R}^{q} j_{*} \mathcal{A}\right) \Rightarrow \underline{\lim } H^{p+q}\left(B_{W_{L / K, S}}, \mathcal{A}_{L, S}\right) .
$$

If $\mathcal{A}$ is represented by a continuous discrete $W_{K}$-module $A$ or by the topological group $\mathbb{R}$ with trivial action, then we have a spectral sequence

$$
\stackrel{H}{\rightarrow}^{p}\left(\mathfrak{F}_{L / K, S}, \underline{R}^{q} j_{*} A\right) \Rightarrow H^{p+q}\left(B_{W_{K}}, A\right) .
$$

Proof. The composition $B_{L / K, S} \rightarrow \mathfrak{F}_{L / K, S} \rightarrow \underline{\text { Set }}$ yields a Leray spectral sequence

$$
H^{p}\left(\mathfrak{F}_{L / K, S}, R^{q}\left(j_{L, S, *}\right) \mathcal{A}_{L, S}\right) \Rightarrow H^{p+q}\left(B_{W_{L / K, S}}, \mathcal{A}_{L, S}\right) .
$$

Passing to the limit (which is valid thanks to the previous lemma), we get the first spectral sequence of the proposition. By 4, Lemma 10, one has

$$
\lim _{L, S} H^{p}\left(B_{W_{L / K, S}}, A^{N_{L, S}}\right)=H^{p}\left(B_{W_{K}}, A\right)
$$

for $A=\mathbb{R}$ or $A$ a continuous discrete $W_{K}$-module. This yields the second spectral sequence of the proposition.

4.2. Computation of the Weil-étale cohomology. In what follows, we consider a non-trivial valuation $v$ of $K$.

Notation 4.7. We denote by $W_{K_{v}}^{1}$ the maximal compact sub-group of $W_{K_{v}}$. Hence $W_{K_{v}}^{1}=I_{v}$ is the inertia sub-group for ultrametric $v$ and $W_{K_{v}}^{1} \simeq \mathbb{S}^{1}$ for complex $v$. Let $\widetilde{W}_{K_{v}}^{1}$ be the image of $W_{K_{v}}^{1}$ in $W_{L / K, S}$. 
Consider the commutative square

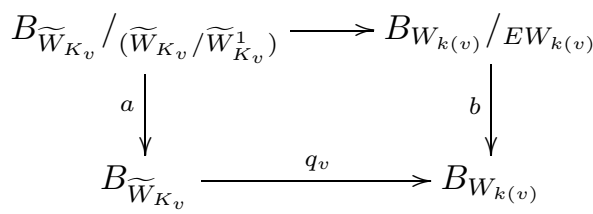

The first horizontal arrow is just (see [5], IV.5.8)

$$
e_{\widetilde{W}_{K_{v}}^{1}}: B_{\widetilde{W}_{K_{v}}^{1}} \simeq B_{\widetilde{W}_{K_{v}}} /\left(\widetilde{W}_{K_{v}} / \widetilde{W}_{K_{v}}^{1}\right) \longrightarrow B_{W_{k(v)}} / E W_{k(v)} \simeq \mathcal{T}
$$

The morphisms $a$ and $b$ are the localization morphisms, and this square is a pullback (see [5], IV.5.8). It follows that the natural transformation

$$
b^{*} \circ q_{v *} \rightarrow e_{\widetilde{W}_{K_{v}}^{1} *} \circ a^{*}
$$

is an isomorphism. The localization functors $a^{*}$ and $b^{*}$ are both exact and preserve injective objects. We get

$$
b^{*} \circ R^{n}\left(q_{v *}\right) \simeq R^{n}\left(e_{\widetilde{W}_{K_{v}}^{1}}\right) \circ a^{*} .
$$

In other words, $R^{n}\left(q_{v *}\right) \mathcal{A}$ is the group object $R^{n}\left(e_{\widetilde{W}_{K_{v}}^{1} *}\right)$ of $\mathcal{T}$ endowed with its natural action of $y\left(W_{k(v)}\right)$, since the functor $b^{*}: B_{W_{k(v)}} \rightarrow \mathcal{T}$ is the forgetful functor (sending an object $\mathcal{F}$ endowed with an action of $y\left(W_{k(v)}\right)$ to $\mathcal{F}$ ). Following the notation of [4], we denote by $\underline{H}^{n}\left(\widetilde{W}_{K_{v}}^{1}, \mathcal{A}\right)$ the object $R^{n}\left(q_{v *}\right) \mathcal{A}$ of $B_{W_{k(v)}}$. We have

$$
R^{q}\left(j_{L, S, *}\right) \mathcal{A}=\left(i_{v *}\right)_{v \neq v_{0}}\left(\underline{H}^{q}\left(\widetilde{W}_{K_{v}}^{1}, \mathcal{A}\right)_{v \neq v_{0}}\right),
$$

for any abelian object $\mathcal{A}$ of $B_{W_{L / K, S}}$ and any $q \geq 1$, as it follows from Proposition 4.1 and from the discussion above. But the direct image functor

$$
\left(i_{v *}\right)_{v \neq v_{0}}: \coprod_{v \neq v_{0}} B_{W_{k(v)}} \longrightarrow \mathfrak{F}_{L / K, S}
$$

is exact (as it follows from its explicit description) and preserves injective objects. Therefore one has

$$
\begin{aligned}
H^{p}\left(\mathfrak{F}_{L / K, S}, R^{q}\left(j_{L, S, *}\right) \mathcal{A}\right) & =H^{p}\left(\coprod_{v \neq v_{0}} B_{W_{k(v)}}, \underline{H}^{q}\left(\widetilde{W}_{K_{v}}^{1}, \mathcal{A}\right)_{v \neq v_{0}}\right) \\
& =\prod_{v \neq v_{0}} H^{p}\left(B_{W_{k(v)}}, \underline{H}^{q}\left(\widetilde{W}_{K_{v}}^{1}, \mathcal{A}\right)\right) .
\end{aligned}
$$

If $v$ is not in $S$, then $\widetilde{W}_{K_{v}}^{1}=0$ (see [6], Lemma 3.7). We get $\left.\underline{H}^{q}\left(\widetilde{W}_{K_{v}}^{1}, \mathcal{A}\right)\right)=0$ for $v$ not in $S$. The next result follows.

Proposition 4.8. For any abelian object $\mathcal{A}$ of $B_{W_{L / K, S}}$ and any $q \geq 1$, we have

$$
H^{p}\left(\mathfrak{F}_{L / K, S}, R^{q}\left(j_{L, S, *}\right) \mathcal{A}\right)=\prod_{v \in S} H^{p}\left(B_{W_{k(v)}}, \underline{H}^{q}\left(\widetilde{W}_{K_{v}}^{1}, \mathcal{A}\right)\right) .
$$


By [4, Proposition 9.2, the sheaf $\underline{H}^{q}\left(\widetilde{W}_{K_{v}}^{1}, \mathbb{Z}\right)$ is represented by the discrete $W_{k(v)}$-module $H^{q}\left(\widetilde{W}_{K_{v}}^{1}, \mathbb{Z}\right)$. Using [4, Proposition 8.1, we get

$$
\begin{aligned}
\stackrel{H}{P}^{p}\left(\mathfrak{F}_{L / K, S}, \underline{R}^{q} j_{*} \mathbb{Z}\right) & =\stackrel{\lim }{\longrightarrow} L, S_{v \in S} \sum_{v \in} H^{p}\left(W_{k(v)}, H^{q}\left(\widetilde{W}_{K_{v}}^{1}, \mathbb{Z}\right)\right) \\
& =\sum_{v \neq v_{0}} H^{p}\left(W_{k(v)}, H^{q}\left(W_{K_{v}}^{1}, \mathbb{Z}\right)\right),
\end{aligned}
$$

where the $(L / K, S)$ runs over the set of finite Galois extensions and finite $S$. We have obtained the next corollary.

Corollary 4.9. For any $q \geq 1$, we have the following identifications:

$$
\begin{aligned}
& H^{p}\left(\mathfrak{F}_{W ; \bar{Y}}, R^{q}\left(j_{*}\right) \mathbb{Z}\right)=\prod_{v \neq v_{0}} H^{p}\left(W_{k(v)}, H^{q}\left(W_{K_{v}}^{1}, \mathbb{Z}\right)\right), \\
& \stackrel{H}{\rightarrow}^{p}\left(\mathfrak{F}_{L / K, S}, \underline{R}^{q} j_{*} \mathbb{Z}\right)=\sum_{v \neq v_{0}} H^{p}\left(W_{k(v)}, H^{q}\left(W_{K_{v}}^{1}, \mathbb{Z}\right)\right) .
\end{aligned}
$$

Let $\operatorname{Pic}(\bar{Y})$ be the Arakelov Picard group of $K$. This is the group obtained by taking the idèle group of $K$ and dividing it by the principal idèles and the unit idèles. We denote by $\operatorname{Pic}(Y)$ the class group of $K$. Let $\operatorname{Pic}^{1}(\bar{Y})$ be the kernel of the absolute value map from $\operatorname{Pic}(\bar{Y})$ to $\mathbb{R}^{>0}$. One has an exact sequence of abelian compact groups

$$
0 \rightarrow \mathbb{R}^{r_{1}+r_{2}-1} / \log \left(U_{K} / \mu_{K}\right) \rightarrow \operatorname{Pic}^{1}(\bar{Y}) \rightarrow \operatorname{Pic}(Y) \rightarrow 0,
$$

where $\log \left(U_{K} / \mu_{K}\right)$ denotes the image of the logarithmic embedding of the units modulo torsion $U_{K} / \mu_{K}$ in the kernel $\mathbb{R}^{r_{1}+r_{2}-1}$ of the sum map $\Sigma: \mathbb{R}^{r_{1}+r_{2}} \rightarrow \mathbb{R}$. By Pontryagin duality, we obtain an exact sequence of discrete abelian groups (see also [6], Proposition 6.4):

$$
0 \rightarrow \operatorname{Pic}(Y)^{\mathcal{D}} \rightarrow \operatorname{Pic}^{1}(\bar{Y})^{\mathcal{D}} \rightarrow \operatorname{Hom}\left(U_{K}, \mathbb{Z}\right) \rightarrow 0 .
$$

Theorem 4.10. One has

$$
\begin{aligned}
\stackrel{H}{\rightarrow}^{n}\left(\mathfrak{F}_{L / K, S}, \mathbb{Z}\right) & =\mathbb{Z} \text { for } n=0 \\
& =0 \text { for } n=1 \\
& =\operatorname{Pic}^{1}(\bar{Y})^{\mathcal{D}} \text { for } n=2 \\
& =\mu_{K}^{\mathcal{D}} \text { for } n=3 .
\end{aligned}
$$

The group $\underline{H}^{n}\left(\mathfrak{F}_{L / K, S}, \mathbb{Z}\right)$ is of infinite rank for even $n \geq 4$ and vanishes for odd $n \geq 5$.

Proof. The cohomology of $W_{K_{v}}^{1}$ is given by class field theory for $v$ ultrametric. For a complex valuation $v$, we have $H^{q}\left(W_{K_{v}}^{1}, \mathbb{Z}\right)=H^{q}\left(\mathbb{S}^{1}, \mathbb{Z}\right)$, which is $\mathbb{Z}$ for $q$ even and 0 for $q$ odd (this follows from [4], Prop. 5.2 and from the fact that the classifying space of $\mathbb{S}^{1}$ is $\mathbb{C} P^{\infty}$ ). Moreover, one has $H^{p}\left(B_{\mathbb{R}}, \mathbb{Z}\right)=0$ for any $q \geq 1$ (see [4, Proposition 9.6). Therefore, Corollary 4.9 yields

$$
\begin{aligned}
\underline{H}^{p}\left(\mathfrak{F}_{L / K, S}, R^{q}\left(j_{*}\right) \mathbb{Z}\right) & =\sum_{v \neq v_{0}, v \nmid \infty}\left(\mathcal{O}_{K_{v}}^{\times}\right)^{\mathcal{D}} \oplus \sum_{v \mid \infty} \mathbb{Z} \text { for } p=0 \text { and } q=2 \\
& =\sum_{v \mid \infty} \mathbb{Z} \text { for } p=0 \text { and } q \geq 4 \text { even } \\
& =0 \text { otherwise. }
\end{aligned}
$$


Now the second spectral sequence of Proposition 4.6 for $A=\mathbb{Z}$ gives

$$
\stackrel{H}{\rightarrow}^{1}\left(\mathfrak{F}_{L / K, S}, \mathbb{Z}\right)=H^{1}\left(W_{K}, \mathbb{Z}\right)=0 .
$$

Next, we obtain the exact sequence

$$
0 \rightarrow \underline{H}^{2}\left(\mathfrak{F}_{L / K, S}, \mathbb{Z}\right) \rightarrow H^{2}\left(W_{K}, \mathbb{Z}\right) \rightarrow \sum_{v \nmid \infty}\left(\mathcal{O}_{K_{v}}^{\times}\right)^{\mathcal{D}} \oplus \sum_{v \mid \infty} \mathbb{Z} \rightarrow \underline{H}^{3}\left(\mathfrak{F}_{L / K, S}, \mathbb{Z}\right) \rightarrow 0 .
$$

This is the Pontryagin dual of

$$
0 \rightarrow \underline{H}^{3}\left(\mathfrak{F}_{L / K, S}, \mathbb{Z}\right)^{\mathcal{D}} \rightarrow \prod_{v \nmid \infty} \mathcal{O}_{K_{v}}^{\times} \times \prod_{v \mid \infty} \mathbb{S}^{1} \rightarrow C_{K}^{1} \rightarrow \underline{H}^{2}\left(\mathfrak{F}_{L / K, S}, \mathbb{Z}\right)^{\mathcal{D}} \rightarrow 0 .
$$

The result for $n \leq 3$ follows. Furthermore, the spectral sequence provides us with the exact sequence

$$
0 \rightarrow \underline{H}^{n}\left(\mathfrak{F}_{L / K, S}, \mathbb{Z}\right)^{\mathcal{D}} \rightarrow H^{n}\left(W_{K}, \mathbb{Z}\right) \rightarrow \bigoplus_{v \mid \infty} \mathbb{Z} \rightarrow \underline{H}^{n+1}\left(\mathfrak{F}_{L / K, S}, \mathbb{Z}\right) \rightarrow 0,
$$

for even $n \geq 4$. Therefore, the result for $n \geq 4$ follows from the fact that the map $H^{n}\left(W_{K}, \mathbb{Z}\right) \rightarrow \bigoplus_{v \mid \infty} \mathbb{Z}$ is surjective (see [4, proof of Corollary 9 ).

Theorem 4.11. For $n \leq 1$ and $n \geq 4$, the canonical map

$$
\underline{H}^{n}\left(\mathfrak{F}_{L / K, S}, \mathbb{Z}\right) \longrightarrow H^{n}\left(\mathfrak{F}_{W, \bar{Y}}, \mathbb{Z}\right)
$$

is an isomorphism. Furthermore, there is an exact sequence

$$
0 \rightarrow H^{2}\left(\mathfrak{F}_{W, \bar{Y}}, \mathbb{Z}\right) \rightarrow\left(C_{K}^{1}\right)^{\mathcal{D}} \rightarrow \prod_{v \nmid \infty}\left(\mathcal{O}_{K_{v}}^{\times}\right)^{\mathcal{D}} \times \prod_{v \mid \infty} \mathbb{Z} \rightarrow H^{3}\left(\mathfrak{F}_{W, \bar{Y}}, \mathbb{Z}\right) \rightarrow 0 .
$$

In particular, the canonical map

$$
\underline{H}^{n}\left(\mathfrak{F}_{L / K, S}, \mathbb{Z}\right) \longrightarrow H^{n}\left(\mathfrak{F}_{W, \bar{Y}}, \mathbb{Z}\right)
$$

is not an isomorphism for $n=2,3$.

Proof. The morphism of topoi $\mathfrak{F}_{W, \bar{Y}} \rightarrow \mathfrak{F}_{L / K, S}$ yields a map $H^{n}\left(\mathfrak{F}_{L / K, S}, \mathbb{Z}\right) \rightarrow$ $H^{n}\left(\mathfrak{F}_{W, \bar{Y}}, \mathbb{Z}\right)$. By the universal property of the inductive limit we get a morphism

$$
\underline{H}^{n}\left(\mathfrak{F}_{L / K, S}, \mathbb{Z}\right) \longrightarrow H^{n}\left(\mathfrak{F}_{W, \bar{Y}}, \mathbb{Z}\right) .
$$

Using Proposition 3.14 (with $L^{\prime}=\bar{K}$ ) and passing to the limit, we obtain a morphism of spectral sequences

$$
\left.\stackrel{H}{\rightarrow}^{p}\left(\mathfrak{F}_{L / K, S}, \underline{R}^{q} j_{*} \mathbb{Z}\right) \Rightarrow H^{p}\left(B_{W_{K}}, \mathbb{Z}\right)\right] \longrightarrow\left[H^{p}\left(\mathfrak{F}_{W, \bar{Y}}, R^{q}\left(j_{*}\right) \mathbb{Z}\right) \Rightarrow H^{p}\left(B_{W_{K}}, \mathbb{Z}\right)\right] .
$$

Comparing these spectral sequences and using the previous proof, we deduce the result.

Let $\bar{V}=\left(V, V_{\infty}\right)$ be an open sub-scheme of $\bar{Y}$. It defines a sub-object of the final object of $\mathfrak{F}_{L / K, S}$. The open sub-topos

$$
\mathfrak{F}_{L / K, S} / \bar{V} \longrightarrow \mathfrak{F}_{L / K, S}
$$

is the full sub-category of $\mathfrak{F}_{L / K, S}$ whose objects are of the form $\mathcal{F}=\left(F_{v} ; f_{v}\right)_{v \in \bar{V}}$ (i.e. $F_{v}=\emptyset$ for $v \in \bar{Y}-\bar{V}$ ).

Definition 4.12. We denote by $H^{n}\left(\mathfrak{F}_{L / K, S}, \bar{V},-\right):=H^{n}\left(\mathfrak{F}_{L / K, S} / \bar{V},-\right)$ the cohomology of the open sub-topos $\mathfrak{F}_{L / K, S} / \bar{V}$. Then we set

$$
\underline{H}^{n}\left(\mathfrak{F}_{L / K, S}, \bar{V}, \mathbb{Z}\right)=\underline{\lim } H^{n}\left(\mathfrak{F}_{L / K, S}, \bar{V}, \mathbb{Z}\right) .
$$


Let $L / K$ be a finite extension of $K$. For any place $v$ of $L$, we denote by $U_{L_{v}}$ the maximal compact sub-group of $L_{v}^{\times}$. Hence $U_{L_{v}}=\mathcal{O}_{L_{v}}^{\times}$for a finite place $v, U_{L_{v}}=\mathbb{S}^{1}$ for $v$ complex and $U_{L_{v}}=\mathbb{Z} / 2 \mathbb{Z}$ for a real place $v$.

Definition 4.13. Let $\bar{V}=\left(V, V_{\infty}\right)$ be a connected étale $\bar{Y}$-scheme and let $L=$ $K(\bar{V})$ be the number field corresponding to the generic point of $\bar{V}$. We define the class group $C_{\bar{V}}$ associated to $\bar{V}$ by the exact sequence of topological groups

$$
0 \rightarrow \prod_{v \in \bar{V}} U_{L_{v}} \longrightarrow C_{L} \rightarrow C_{\bar{V}} \rightarrow 0
$$

We denote by $C_{\bar{V}}^{1}$ the topological sub-group of $C_{\bar{V}}$ defined by the kernel of the canonical continuous morphism $C_{\bar{V}} \rightarrow \mathbb{R}_{>0}$.

Note that $C_{\bar{V}}$ is just the $S$-idèle class group of the number field $K(\bar{V})$, where $S$ is the set of valuations of $K(\bar{V})$ not corresponding to a point of $\bar{V}$. The group $C_{\bar{V}}^{1}$ is compact.

Proposition 4.14. Let $\bar{V} \varsubsetneqq \bar{Y}$ be a proper open sub-scheme of $\bar{Y}$. Then one has

$$
\begin{aligned}
\stackrel{H}{\rightarrow}^{n}\left(\mathfrak{F}_{L / K, S}, \bar{V}, \mathbb{Z}\right) & =\mathbb{Z} \text { for } n=0 \\
& =0 \text { for } n \text { odd } \\
& =\left(C_{\bar{V}}^{1}\right)^{\mathcal{D}} \text { for } n=2 .
\end{aligned}
$$

For even $n \geq 4$, we have an exact sequence

$$
0 \longrightarrow \underline{H}^{n}\left(\mathfrak{F}_{L / K, S}, \bar{V}, \mathbb{Z}\right) \longrightarrow H^{n}\left(W_{K}, \mathbb{Z}\right) \longrightarrow \sum_{v \in V_{\infty}} \mathbb{Z} \longrightarrow 0 .
$$

Proof. We again use the Leray spectral sequence induced by the inclusion of the generic point. We get $\underline{H}^{1}\left(\mathfrak{F}_{L / K, S}, \bar{V}, \mathbb{Z}\right)=H^{1}\left(W_{K}, \mathbb{Z}\right)=0$ and the exact sequence

$0 \rightarrow \underline{H}^{2}\left(\mathfrak{F}_{L / K, S}, \bar{V}, \mathbb{Z}\right) \rightarrow\left(C_{K}^{1}\right)^{\mathcal{D}} \rightarrow \coprod_{v \in V}\left(\mathcal{O}_{K_{v}}^{\times}\right)^{\mathcal{D}} \oplus \coprod_{v \in V_{\infty}} \mathbb{Z} \rightarrow \underline{H}^{3}\left(\mathfrak{F}_{L / K, S}, \bar{V}, \mathbb{Z}\right) \rightarrow 0$.

Moreover the map

$$
\prod_{v \in \bar{V}} U_{K_{v}}=\prod_{v \in V} \mathcal{O}_{K_{v}}^{\times} \times \prod_{v \in V_{\infty}} \mathbb{S}^{1} \longrightarrow C_{K}^{1}
$$

is injective, since $\bar{V} \varsubsetneqq \bar{Y}$. By Pontryagin duality, we obtain $\underline{H}^{3}\left(\mathfrak{F}_{L / K, S}, \bar{V}, \mathbb{Z}\right)=0$ and

$$
\underline{H}^{2}\left(\mathfrak{F}_{L / K, S}, \bar{V}, \mathbb{Z}\right)=\operatorname{Ker}\left[\left(C_{K}^{1}\right)^{\mathcal{D}} \rightarrow \coprod_{v \in \bar{V}} U_{K_{v}}^{\mathcal{D}}\right]=\left(C_{\bar{V}}^{1}\right)^{\mathcal{D}}
$$

Next we obtain an exact sequence

$$
\begin{aligned}
0 & \rightarrow \underline{H}^{n}\left(\mathfrak{F}_{L / K, S}, \bar{V}, \mathbb{Z}\right) \rightarrow H^{n}\left(W_{K}, \mathbb{Z}\right) \rightarrow \sum_{v \in V_{\infty}} \mathbb{Z} \\
& \rightarrow \underline{H}^{n+1}\left(\mathfrak{F}_{L / K, S}, \bar{V}, \mathbb{Z}\right) \rightarrow H^{n+1}\left(W_{K}, \mathbb{Z}\right)=0
\end{aligned}
$$

for even $n \geq 4$. This ends the proof since the map $H^{n}\left(W_{K}, \mathbb{Z}\right) \rightarrow \sum_{v \in V_{\infty}} \mathbb{Z}$ is surjective for even $n \geq 4$ (see [4], proof of Corollary 9).

Proposition 4.15. For any open sub-scheme $\bar{V} \subseteq \bar{Y}$, one has

$$
\underline{H}^{n}\left(\mathfrak{F}_{L / K, S}, \bar{V}, \widetilde{\mathbb{R}}\right)=\mathbb{R} \text { for } n=0,1 \text { and } \underline{H}^{n}\left(\mathfrak{F}_{L / K, S}, \bar{V}, \widetilde{\mathbb{R}}\right)=0 \text { for } n \geq 2 \text {. }
$$


Proof. Arguing as above and using the fact that $H^{n}\left(W_{K_{v}}^{1}, \widetilde{\mathbb{R}}\right)=0$ for $n \geq 1$ and any non-trivial valuation $v$ since $W_{K_{v}}^{1}$ is compact (see 4, Corollary 8), we obtain $H^{n}\left(\mathfrak{F}_{L / K, S}, \bar{V}, \widetilde{\mathbb{R}}\right)=H^{n}\left(W_{L / K, S}, \widetilde{\mathbb{R}}\right)$ for any $\bar{V} \subseteq \bar{Y}$ and any pair $(L, S)$. Now the product decomposition $W_{L / K, S}=W_{L / K, S}^{1} \times \mathbb{R}$ and the compactness of $W_{L / K, S}^{1}$ show (see [4, Proposition 9.6):

$$
H^{n}\left(W_{L / K, S}, \widetilde{\mathbb{R}}\right)=H^{n}(\mathbb{R}, \widetilde{\mathbb{R}})=\mathbb{R} \text { for } n=0,1 \text { and } 0 \text { for } n \geq 2 .
$$

Passing to the limit over $(L, S)$, we obtain the result.

4.3. Cohomology with compact support. The open sub-topos $\phi: \mathfrak{F}_{L / K, S} / Y \rightarrow$ $\mathfrak{F}_{L / K, S}$ associated to the inclusion $Y \rightarrow \bar{Y}$ gives rise to three adjoint functors $\phi_{L, S, !}, \phi_{L, S}^{*}, \phi_{L, S, *}$. The functor $\phi_{L, S, !}$ is the usual extension by 0 . If $\mathcal{A}=\left(A_{v} ; f_{v}\right)_{v \in Y}$ is an abelian object of $\mathfrak{F}_{L / K, S} / Y$, then $\phi_{L, S, !} \mathcal{A}$ is the abelian object of $\mathfrak{F}_{L / K, S}$ whose $v$-component is $A_{v}$ for $v \in Y$ and 0 for $v \in Y_{\infty}$. If there is no risk of ambiguity, we denote by $\phi_{!}, \phi^{*}, \phi_{*}$ the functors defined above.

For any abelian object $\mathcal{A}=\left(A_{v} ; f_{v}\right)_{v \in \bar{Y}}$ of $\mathfrak{F}_{L / K, S}$ we have an exact sequence

$$
0 \rightarrow \phi_{!} \phi^{*} \mathcal{A} \rightarrow \mathcal{A} \rightarrow \prod_{Y_{\infty}} i_{v, *} A_{v} \rightarrow 0 .
$$

Moreover, $i_{v, *}$ is exact and preserves injectives; hence one has

$$
H^{n}\left(\mathfrak{F}_{L / K, S}, \prod_{Y_{\infty}} i_{v, *} A_{v}\right)=H^{n}\left(\coprod_{Y_{\infty}} B_{\mathbb{R}}, \prod_{Y_{\infty}} i_{v, *} A_{v}\right)=\prod_{Y_{\infty}} H^{n}\left(B_{\mathbb{R}}, A_{v}\right) .
$$

Notation 4.16 . We denote by $\phi_{!} \mathbb{Z}$ (respectively $\phi_{!} \widetilde{\mathbb{R}}$ ) the abelian object of $T o p\left(\mathfrak{F}_{\bullet}\right)$ defined by the family of sheaves $\phi_{L, S, !} \mathbb{Z}$ (respectively $\phi_{L, S, !} \widetilde{\mathbb{R}}$ ) with the obvious transition maps.

Using the exact sequence of sheaves (6), equation (7) and passing to the limit we get the following long exact sequences for any open sub-scheme $\bar{V} \subseteq \bar{Y}$ :

$$
\begin{aligned}
& \ldots \rightarrow \underline{H}^{n}\left(\mathfrak{F}_{L / K, S}, \bar{V}, \phi ! \mathbb{Z}\right) \rightarrow \underline{H}^{n}\left(\mathfrak{F}_{L / K, S}, \bar{V}, \mathbb{Z}\right) \rightarrow \prod_{V_{\infty}} H^{n}\left(B_{\mathbb{R}}, \mathbb{Z}\right) \rightarrow \ldots, \\
& \ldots \rightarrow \underline{H}^{n}\left(\mathfrak{F}_{L / K, S}, \bar{V}, \phi, \widetilde{\mathbb{R}}\right) \rightarrow \underline{H}^{n}\left(\mathfrak{F}_{L / K, S}, \bar{V}, \widetilde{\mathbb{R}}\right) \rightarrow \prod_{V_{\infty}} H^{n}\left(B_{\mathbb{R}}, \widetilde{\mathbb{R}}\right) \rightarrow \ldots
\end{aligned}
$$

By $\left(\left[4\right.\right.$, Proposition 9.6), we have $H^{n}\left(B_{\mathbb{R}}, \mathbb{Z}\right)=0$ for any $n \geq 1, H^{n}\left(B_{\mathbb{R}}, \widetilde{\mathbb{R}}\right)=\mathbb{R}$ for $n=0,1$ and $H^{n}\left(B_{\mathbb{R}}, \widetilde{\mathbb{R}}\right)=0$ for $n \geq 2$. This is enough to compute the groups $\underline{H}^{n}\left(\mathfrak{F}_{L / K, S}, \bar{V}, \phi ! \mathbb{Z}\right)$ and $\underline{H}^{n}\left(\mathfrak{F}_{L / K, S}, \bar{V}, \phi_{!} \widetilde{\mathbb{R}}\right)$ for any open $\bar{V} \subseteq \bar{Y}$. In particular, we recover the result ([], Theorem 6.3) for $\bar{V}=\bar{Y}$ and $n \leq 3$.

\section{The CAtegory of Sheaves on Lichtenbaum's Weil-Étale Site}

In this section we show that the topos $\mathfrak{F}_{L / K, S}$ is equivalent to the category of sheaves on Lichtenbaum's Weil-étale site $T_{L / K, S}$.

5.1. The local section site. The Weil-étale site $\left(T_{L / K, S}, \mathcal{J}_{l s}\right)$ is defined in [6] using the groups $W_{L / K ; S}$, where $L / K$ is a finite Galois extension and $S$ a finite set of primes of $K$ containing the archimedean ones and the primes ramified in $L / K$. 
Definition 5.1. An object of the category $T_{L / K, S}$ is a collection $\mathcal{X}=\left(X_{v} ; f_{v}\right)_{v \in \bar{Y}}$, where $X_{v}$ is a $W_{k(v)}$-topological space and $f_{v}: X_{v} \rightarrow X_{v_{0}}$ is a morphism of $W_{K_{v}}{ }^{-}$ spaces for any $v \neq v_{0}$ (the topological group $W_{K_{v}}$ acts continuously on $X_{v}$ and $X_{v_{0}}$ via the morphisms $\theta_{v}: W_{K_{v}} \rightarrow W_{K}$ and $q_{v}: W_{K_{v}} \rightarrow W_{k(v)}$ respectively). If $v=v_{0}$ we require that the action of $W_{K}$ on $X_{v_{0}}$ factors through $W_{L / K, S}$.

The morphisms in this category are defined in the obvious way. The topology $\mathcal{J}_{l s}$ on the category $T_{L / K, S}$ is generated by the pre-topology for which a cover is a family of morphisms $\left\{\mathcal{X}_{i} \rightarrow \mathcal{X}\right\}$ such that $\left\{X_{i ; v} \rightarrow X_{v}\right\}$ is a local section cover, for any valuation $v$.

M. Flach has shown in 4 that the definition of $H^{i}\left(W_{K} ; A\right)$ as the direct limit $\stackrel{\lim }{\rightarrow} H^{i}\left(W_{L / K ; S} ; A\right)$ coincides with the topological group cohomology of $W_{K}$. Here,

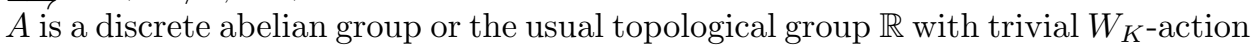
(see 4], Lemma 10). This suggests the following definition.

Definition 5.2. The local section site $\left(T_{W: \bar{Y}} ; \mathcal{J}_{l s}\right)$ is defined as above, but the action of $W_{K}$ on the generic component $X_{v_{0}}$ of an object $\mathcal{X}$ of $T_{W ; \bar{Y}}$ is not supposed to factor through $W_{L / K, S}$.

For any topological group $G$, we denote by $B_{T o p} G$ the category of topological spaces (in a given universe) on which $G$ acts continuously. The functor $B_{T o p} \widetilde{W}_{K_{v}} \rightarrow$ $B_{T o p} W_{K_{v}}$ induced by the surjective map $W_{K_{v}} \rightarrow \widetilde{W}_{K_{v}}$ is fully faithful. Therefore, an object of the category $T_{L / K, S}$ is given by a collection $\mathcal{X}=\left(X_{v} ; f_{v}\right)_{v \in \bar{Y}}$, where $f_{v}$ is a map of $\widetilde{W}_{K_{v}}$-topological spaces.

The category $T_{W ; \bar{Y}}$ (respectively $T_{L / K, S}$ ) has finite projective limits. Indeed, the final object is given by the trivial action of $W_{k(v)}$ on the one point space $X_{v}:=\{*\}$ for any $v$ and by the trivial map $f_{v}: X_{v} \rightarrow X_{v_{0}}$. Let

$$
\phi: \mathcal{U}=\left(U_{v} ; f_{v}\right) \longrightarrow \mathcal{X}=\left(X_{v} ; \xi_{v}\right) \text { and } \phi^{\prime}: \mathcal{U}^{\prime}=\left(U_{v}^{\prime} ; f_{v}^{\prime}\right) \rightarrow \mathcal{X}=\left(X_{v} ; \xi_{v}\right)
$$

be two morphisms in $T_{W ; \bar{Y}}$ (respectively in $\left.T_{L / K, S}\right)$. The object $\left(U_{v} \times_{X_{v}} U_{v}^{\prime} ; f_{v} \times_{\xi_{v}}\right.$ $\left.f_{v}^{\prime}\right)_{v \in Y}$ does define a fiber product $\mathcal{U} \times \mathcal{X} \mathcal{U}^{\prime}$ in the category $T_{W ; \bar{Y}}$ (respectively $\left.T_{L / K, S}\right)$.

\subsection{The local section site is a site for the flask topos.}

Notation 5.3. In order to simplify the notation, we assume in this subsection that $L=\bar{K} / K$ is an algebraic closure of $K$ and $S$ is the set of all places of $K$. However, everything here remains valid for any suitable pair $(L / K, S)$.

Let $v$ be a valuation of $K$. The Yoneda embedding yields fully faithful functors

$$
\varepsilon_{v}: B_{T o p} W_{k(v)} \rightarrow B_{W_{k(v)}} \text { and } \varepsilon_{K_{v}}: B_{T o p} W_{K_{v}} \rightarrow B_{W_{K_{v}}} .
$$

Recall that $\theta_{v}^{*}: B_{W_{K}} \rightarrow B_{W_{K v}}$ and $q_{v}^{*}: B_{W_{k(v)}} \rightarrow B_{W_{K v}}$ denote the pull-backs of the morphisms of classifying topoi induced by the Weil map $\theta_{v}: W_{K_{v}} \rightarrow W_{K}$ and by the projection $q_{v}: W_{K_{v}} \rightarrow W_{k(v)}$. In the following proof, we also denote by

$$
{ }^{t} \theta_{v}^{*}: B_{T o p} W_{K} \rightarrow B_{T o p} W_{K_{v}} \text { and }{ }^{t} q_{v}^{*}: B_{T o p} W_{k(v)} \rightarrow B_{T o p} W_{K_{v}}
$$

the functors induced by $\theta_{v}$ and $q_{v}$. One has

$$
q_{v}^{*} \circ \varepsilon_{v}=\varepsilon_{K_{v}} \circ{ }^{t} q_{v}^{*} \text { and } \theta_{v}^{*} \circ \varepsilon_{v_{0}}=\varepsilon_{K_{v}} \circ{ }^{t} \theta_{v}^{*} .
$$


Proposition 5.4. There is a fully faithful functor

$$
\begin{aligned}
& \text { y : } \quad T_{W ; \bar{Y}} \longrightarrow \mathfrak{F}_{W ; \bar{Y}}, \\
& \mathcal{X}=\left(X_{v} ; f_{v}\right) \longmapsto \mathrm{y}(\mathcal{X})=\left(\varepsilon_{v}\left(X_{v}\right) ; \varepsilon_{K_{v}}\left(f_{v}\right)\right) \text {. }
\end{aligned}
$$

Proof. If $\mathcal{X}=\left(X_{v} ; f_{v}\right)$ is an object of $T_{W: \bar{Y}}$, then $f_{v}:{ }^{t} q_{v}^{*}\left(X_{v}\right) \rightarrow{ }^{t} \theta_{v}^{*}\left(X_{v_{0}}\right)$ is a map of $B_{T o p} W_{K_{v}}$, for any valuation $v$. By (10), the map

$$
\varepsilon_{K_{v}}\left(f_{v}\right): q_{v}^{*} \circ \varepsilon_{v}\left(X_{v}\right)=\varepsilon_{K_{v}} \circ{ }^{t} q_{v}^{*}\left(X_{v}\right) \longrightarrow \varepsilon_{K_{v}} \circ{ }^{t} \theta_{v}^{*}\left(X_{v_{0}}\right)=\theta_{v}^{*} \circ \varepsilon_{v}\left(X_{v_{0}}\right)
$$

is a morphism of $B_{W_{K_{v}}}$. Hence $\mathrm{y}(\mathcal{X})=\left(\varepsilon_{v}\left(X_{v}\right) ; \varepsilon_{K_{v}}\left(f_{v}\right)\right)$ is an object of $\mathfrak{F}_{W: \bar{Y}}$ and $\mathrm{y}$ is a functor. Let $\mathcal{X}=\left(X_{v} ; f_{v}\right)_{v}$ and $\mathcal{X}^{\prime}=\left(X_{v}^{\prime} ; f_{v}^{\prime}\right)_{v}$ be two objects of $T_{W ; \bar{Y}}$. Denote by

$$
\begin{aligned}
\mathrm{y}_{\left(\mathcal{X} ; \mathcal{X}^{\prime}\right)}: \operatorname{Hom}_{T_{W ; \bar{Y}}}\left(\left(X_{v} ; f_{v}\right)\right. & \left.\left(X_{v}^{\prime} ; f_{v}^{\prime}\right)\right) \\
& \longrightarrow \operatorname{Hom}_{\mathfrak{F}_{W ; \bar{Y}}}\left(\left(\varepsilon_{v} X_{v} ; \varepsilon_{K_{v}} f_{v}\right) ;\left(\varepsilon_{v} X_{v} ; \varepsilon_{K_{v}} f_{v}\right)\right)
\end{aligned}
$$

the map defined by the functor $\mathrm{y}$. One has to show that $\mathrm{y}_{\left(\mathcal{X} ; \mathcal{X}^{\prime}\right)}$ is a bijection, for any objects $\mathcal{X}$ and $\mathcal{X}^{\prime}$. Let

$$
\phi^{\prime}=\left(\phi_{v}^{\prime}\right)_{v}, \phi=\left(\phi_{v}\right)_{v}:\left(X_{v} ; f_{v}\right) \rightrightarrows\left(X_{v}^{\prime} ; f_{v}^{\prime}\right)
$$

be two morphisms in $T_{W ; \bar{Y}}$ such that $\mathrm{y}_{\left(\mathcal{X} ; \mathcal{X}^{\prime}\right)}\left(\phi^{\prime}\right)=\mathrm{y}_{\left(\mathcal{X} ; \mathcal{X}^{\prime}\right)}(\phi)$. One has $\varepsilon_{v}\left(\phi_{v}^{\prime}\right)=$ $\varepsilon_{v}\left(\phi_{v}\right)$ for any $v \in \bar{Y}$. Since $\varepsilon_{v}$ is fully faithful, we get $\phi_{v}^{\prime}=\phi_{v}$; hence the map $\mathrm{y}_{\left(\mathcal{X} ; \mathcal{X}^{\prime}\right)}$ is injective. Let

$$
\varphi=\left(\varphi_{v}\right)_{v}:\left(\varepsilon_{v} X_{v} ; \varepsilon_{K_{v}} f_{v}\right) \rightarrow\left(\varepsilon_{v} X_{v}^{\prime} ; \varepsilon_{K_{v}} f_{v}^{\prime}\right)
$$

be a morphism in $\mathfrak{F}_{W: \bar{Y}}$. For any $v \in \bar{Y}$, there exists a unique morphism $\phi_{v}: X_{v} \rightarrow$ $X_{v}^{\prime}$ such that $\varepsilon_{v}\left(\phi_{v}\right)=\varphi_{v}$ (since $\varepsilon_{v}$ is fully faithful). The square

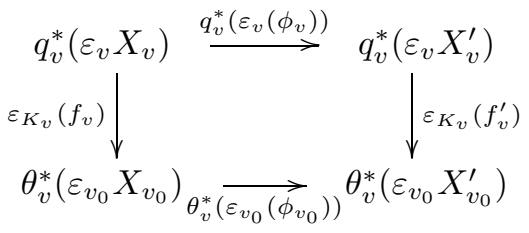

is commutative. By (10), the following diagram commutes as well:

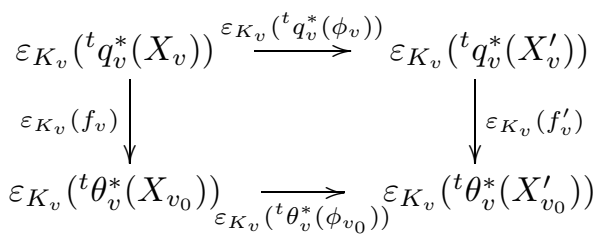

Finally, the square

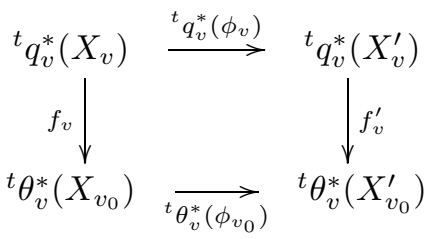

is commutative, since $\varepsilon_{K_{v}}$ is fully faithful. Hence $\phi=\left(\phi_{v}\right)_{v}: \mathcal{X} \rightarrow \mathcal{X}^{\prime}$ is a morphism of $T_{W ; \bar{Y}}$ such that

$$
\mathrm{y}_{\left(\mathcal{X} ; \mathcal{X}^{\prime}\right)}(\phi)=\mathrm{y}_{\left(\mathcal{X} ; \mathcal{X}^{\prime}\right)}\left(\left(\phi_{v}\right)_{v}\right)=\left(\varepsilon_{v} \phi_{v}\right)_{v}=\left(\varphi_{v}\right)_{v}=\varphi .
$$


The functor y is fully faithful, since the map $\mathrm{y}_{\left(\mathcal{X} ; \mathcal{X}^{\prime}\right)}$ is bijective for any objects $\mathcal{X}$ and $\mathcal{X}^{\prime}$ of $T_{W ; \bar{Y}}$.

For the notion of induced topology we refer to ([5], III.3.1).

Proposition 5.5. The local section topology $\mathcal{J}_{l s}$ on $T_{W ; \bar{Y}}$ is the topology induced by the canonical topology of $\mathfrak{F}_{W ; \bar{Y}}$ via the functor $\mathrm{y}$.

Proof. Recall that the coproduct of a family of topoi is, as a category, the product of the underlying categories. Then consider the following commutative diagram:

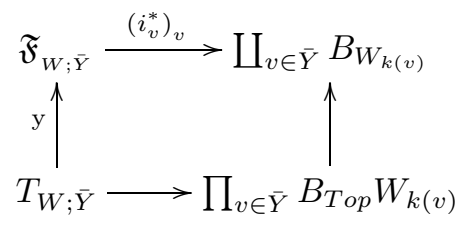

The local section topology on $T_{W ; \bar{Y}}$ is (by definition) the topology induced by the local section topology on $\prod_{v \in \bar{Y}} B_{T o p} W_{k(v)}$ (see [5], III.3.4). By [4], Proposition 4.1, the local section topology on $B_{T o p} W_{k(v)}$ is the topology induced by the canonical topology of $B_{W_{k(v)}}$. Hence the local section topology on $\prod B_{T o p} W_{k(v)}$ is the topology induced by the canonical topology of $\amalg B_{W_{k(v)}}$. Since the previous diagram is commutative, the local section topology on $T_{W ; \bar{Y}}$ is the topology induced by the canonical topology of $\amalg B_{W_{k(v)}}$ via the functor

$$
\left(i_{v}^{*}\right)_{v} \circ \mathrm{y}: T_{W ; \bar{Y}} \longrightarrow \mathfrak{F}_{W ; \bar{Y}} \longrightarrow \coprod_{v \in \bar{Y}} B_{W_{k(v)}} .
$$

Hence, it remains to show that the canonical topology of $\mathfrak{F}_{W ; \bar{Y}}$ is induced by the canonical topology of $\coprod B_{W_{k(v)}}$.

The functor $\left(i_{v}^{*}\right)_{v}: \mathfrak{F}_{W ; \bar{Y}} \rightarrow \amalg B_{W_{k(v)}}$ is the pull-back of the morphism of topoi $\left(i_{v}\right)_{v}: \amalg B_{W_{k(v)}} \rightarrow \mathfrak{F}_{W ; \bar{Y}}$. Then $\left(i_{v}^{*}\right)_{v}$ is a continuous morphism of left exact sites, when $\mathfrak{F}_{W ; \bar{Y}}$ and $\amalg B_{W_{k(v)}}$ are viewed as categories endowed with their canonical topologies. This shows that the topology $\mathcal{J}_{\text {ind }}$ on $\mathfrak{F}_{W: \bar{Y}}$ induced by the canonical topology of $\amalg B_{W_{k(v)}}$ is finer than the canonical topology of $\mathfrak{F}_{W ; \bar{Y}}$, by definition of the induced topology.

We need to show that any representable presheaf is a sheaf on the site $\left(\mathfrak{F}_{W ; \bar{Y}} ; \mathcal{J}_{\text {ind }}\right)$. Let $\mathcal{F}=\left(F_{v} ; f_{v}\right)_{v}$ be an object of $\mathfrak{F}_{W ; \bar{Y}}$, and let

$$
\left\{u_{i}: \mathcal{X}_{i}=\left(X_{i ; v} ; \xi_{i ; v}\right) \longrightarrow \mathcal{X}=\left(X_{v} ; \xi_{v}\right)\right\}_{i \in I}
$$

be a covering family of the site $\left(\mathfrak{F}_{W ; \bar{Y}} ; \mathcal{J}_{\text {ind }}\right)$. The functor

$$
\left(\mathfrak{F}_{W ; \bar{Y}} ; \mathcal{J}_{i n d}\right) \longrightarrow\left(\coprod_{v \in \bar{Y}} B_{W_{k(v)}} ; \mathcal{J}_{c a n}\right) \longrightarrow\left(B_{W_{k(v)}} ; \mathcal{J}_{\text {can }}\right)
$$

is continuous. Therefore (see [5, III.1.6) the family

$$
\left\{u_{i ; v}: X_{i ; v} \longrightarrow X_{v}\right\}_{i \in I}
$$

is a covering family of $\left(B_{W_{k(v)}} ; \mathcal{J}_{\text {can }}\right)$ for any valuation $v$. Since the covering families for the canonical topology of a topos are precisely the epimorphic families, the family $\left\{X_{i ; v} \rightarrow X_{v}\right\}$ is epimorphic. Moreover, the pull-back $q_{v}^{*}$ of the morphism 
$q_{v}: B_{W_{K_{v}}} \rightarrow B_{W_{k(v)}}$ preserves (as any pull-back) epimorphic families. Hence the family

$$
\left\{q_{v}^{*}\left(u_{i ; v}\right): q_{v}^{*}\left(X_{i ; v}\right) \longrightarrow q_{v}^{*}\left(X_{v}\right)\right\}_{i \in I}
$$

is epimorphic in the category $B_{W_{K v}}$. Consider the diagram $\mathbb{D}$ :

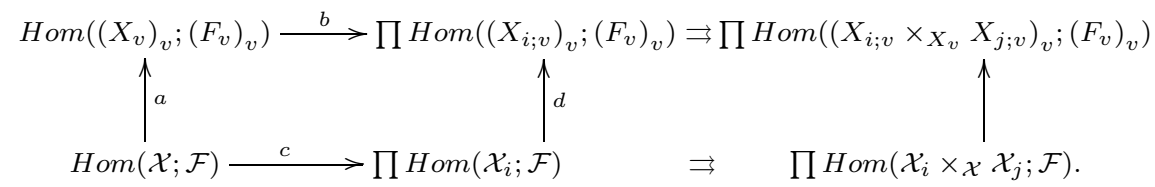

The sets of homomorphisms in the first line correspond to the category $\amalg B_{W_{k(v)}}$, and the set of homomorphisms of the second line correspond to the category $\mathfrak{F}_{W ; \bar{Y}}$. The vertical arrows are given by the faithful functor $\left(i_{v}^{*}\right)_{v}$. Hence those vertical maps are all injective. In particular, $a$ and $d$ are both injective.

The functor $\left(i_{v}^{*}\right)_{v}$ sends covering families of $\mathfrak{F}_{W ; \bar{Y}}$ to covering families of $\amalg B_{W_{k(v)}}$, since $\left(i_{v}^{*}\right)_{v}$ is continuous. Moreover, any representable presheaf of $\amalg B_{W_{k(v)}}$ is a sheaf for the canonical topology. This shows that the first line of $\mathbb{D}$ is exact. Hence, the maps $a$ and $b$ are both injective, which shows that $c$ is injective.

Now, let $\left(\varphi_{i}\right)_{i}$ be an element of the kernel of

$$
\prod \operatorname{Hom}\left(\mathcal{X}_{i} ; \mathcal{F}\right) \rightrightarrows \prod \operatorname{Hom}\left(\mathcal{X}_{i} \times \mathcal{X} \mathcal{X}_{j} ; \mathcal{F}\right)
$$

The square on the right hand side is commutative; hence $d\left(\left(\varphi_{i}\right)_{i}\right)$ is in the kernel of

$$
\prod \operatorname{Hom}\left(\left(X_{i ; v}\right)_{v} ;\left(F_{v}\right)_{v}\right) \rightrightarrows \prod \operatorname{Hom}\left(\left(X_{i ; v} \times_{X_{v}} X_{j ; v}\right)_{v} ;\left(F_{v}\right)_{v}\right)
$$

Then we get an element $\phi \in \operatorname{Hom}\left(\left(X_{v}\right)_{v} ;\left(F_{v}\right)_{v}\right)$ which goes to $d\left(\left(\varphi_{i}\right)_{i}\right)$, since the first line is exact. More precisely, one has $b(\phi)=d\left(\left(\varphi_{i}\right)_{i}\right)$. Let $v$ be a non-trivial valuation. For any $i \in I$, consider the diagram

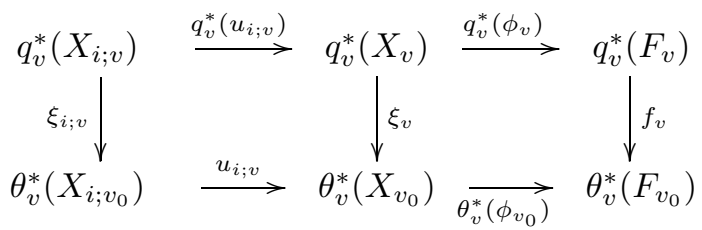

Here, the total square and the left hand side square are both commutative. Indeed,

$$
u_{i} \in \operatorname{Hom}_{\mathfrak{F}_{W ; \bar{Y}}}\left(\mathcal{X}_{i} ; \mathcal{X}\right) \text { and } \phi \circ u_{i}=\varphi_{i} \in \operatorname{Hom}_{\mathfrak{F}_{W ; \bar{Y}}}\left(\mathcal{X}_{i} ; \mathcal{F}\right)
$$

It follows that the elements

$$
\theta_{v}^{*}\left(\phi_{v_{0}}\right) \circ \xi_{v} \text { and } f_{v} \circ q_{v}^{*}\left(\phi_{v}\right)
$$

of the set $\operatorname{Hom}_{B_{W_{K}}}\left(q_{v}^{*}\left(X_{v}\right) ; \theta_{v}^{*}\left(F_{v_{0}}\right)\right)$ have the same image under the morphism

$$
\operatorname{Hom}_{B_{W_{K_{v}}}}\left(q_{v}^{*}\left(X_{v}\right) ; \theta_{v}^{*}\left(F_{v_{0}}\right)\right) \longrightarrow \operatorname{Hom}_{B_{W_{K_{v}}}}\left(q_{v}^{*}\left(X_{i ; v}\right) ; \theta_{v}^{*}\left(F_{v_{0}}\right)\right),
$$

for any $i \in I$. Hence, $\theta_{v}^{*}\left(\phi_{v_{0}}\right) \circ \xi_{v}$ and $f_{v} \circ q_{v}^{*}\left(\phi_{v}\right)$ have the same image under the morphism

$$
\operatorname{Hom}_{B_{W_{K}}}\left(q_{v}^{*}\left(X_{v}\right) ; \theta_{v}^{*}\left(F_{v_{0}}\right)\right) \longrightarrow \operatorname{Hom}_{B_{W_{K}}}\left(\coprod_{i \in I} q_{v}^{*}\left(X_{i ; v}\right) ; \theta_{v}^{*}\left(F_{v_{0}}\right)\right) .
$$


Furthermore, the morphism (11) is injective, since the family $\left\{q_{v}^{*}\left(X_{i ; v}\right) \rightarrow q_{v}^{*}\left(X_{v}\right)\right\}$ is epimorphic. The equality

$$
\theta_{v}^{*}\left(\phi_{v_{0}}\right) \circ \xi_{v}=f_{v} \circ q_{v}^{*}\left(\phi_{v}\right)
$$

follows. For any valuation $v \in \bar{Y}$, the square

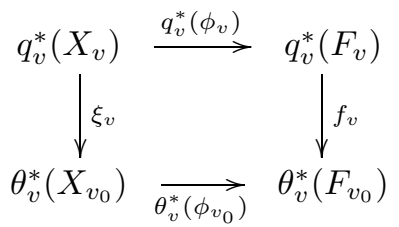

is commutative. In other words, the element $\phi \in \operatorname{Hom}_{\amalg B_{W_{k(v)}}}\left(\left(X_{v}\right)_{v} ;\left(F_{v}\right)_{v}\right)$ lies in

$$
H o m_{\mathfrak{F}_{W ; \bar{Y}}}(\mathcal{X} ; \mathcal{F}) \subseteq \operatorname{Hom}_{\amalg B_{W_{k(v)}}}\left(\left(X_{v}\right)_{v} ;\left(F_{v}\right)_{v}\right) .
$$

Hence there exists a unique $\varphi \in \operatorname{Hom}_{\mathfrak{F}_{W ; \bar{Y}}}(\mathcal{X} ; \mathcal{F})$ such that $a(\varphi)=\phi$. We get

$$
b \circ a(\varphi)=b(\phi)=d\left(\left(\varphi_{i}\right)_{i}\right)=d \circ c(\varphi)
$$

and

$$
c(\varphi)=\left(\varphi_{i}\right)_{i}
$$

since $d$ is injective. This shows that the second line of $\mathbb{D}$ is exact.

We have shown that the sequence

$$
H o m_{\mathfrak{F}_{W ; \bar{Y}}}(\mathcal{X} ; \mathcal{F}) \rightarrow \prod_{i} \operatorname{Hom}_{\mathfrak{F}_{W ; \bar{Y}}}\left(\mathcal{X}_{i} ; \mathcal{F}\right) \rightrightarrows \prod_{i ; j} \operatorname{Hom}_{\mathfrak{F}_{W ; \bar{Y}}}\left(\mathcal{X}_{i} \times \mathcal{X} \mathcal{X}_{j} ; \mathcal{F}\right)
$$

is exact and that the first arrow is injective for any object $\mathcal{F}$ of $\mathfrak{F}_{W ; \bar{Y}}$ and any covering family $\left\{\mathcal{X}_{i} \rightarrow \mathcal{X}\right\}_{i \in I}$ of the site $\left(\mathfrak{F}_{W ; \bar{Y}} ; \mathcal{J}_{\text {ind }}\right)$. Hence any representable presheaf of the category $\mathfrak{F}_{W ; \bar{Y}}$ is a sheaf for the topology $\mathcal{J}_{\text {ind }}$. In other words, the topology $\mathcal{J}_{\text {ind }}$ is sub-canonical, that is, coarser than the canonical topology. Since $\mathcal{J}_{\text {ind }}$ is also finer than the canonical topology, it is the canonical topology.

Corollary 5.6. The functor y is continuous.

Proof. By definition of the induced topology, $\mathcal{J}_{l s}$ is the finest topology on $T_{W ; \bar{Y}}$ such that y is continuous (see [5], III.3.1).

Corollary 5.7. The local section topology $\mathcal{J}_{l s}$ on $T_{W ; \bar{Y}}$ is sub-canonical.

Proof. Let $\mathcal{X}$ be an object of $T_{W ; \bar{Y}}$. The presheaf $\widetilde{\mathrm{y}(\mathcal{X})}$ of $\mathfrak{F}_{W ; \bar{Y}}$ represented by $\mathrm{y}(\mathcal{X})$ is a sheaf, since $\mathfrak{F}_{W ; \bar{Y}}$ is endowed with the canonical topology. The restriction of $\widetilde{\mathrm{y}(\mathcal{X})}$ to the sub-category $T_{W ; \bar{Y}}$ via the functor $\mathrm{y}$ is a sheaf for the local section topology $\mathcal{J}_{l s}$, since $\mathrm{y}$ is continuous. But this sheaf is canonically isomorphic to the presheaf $\widetilde{\mathcal{X}}$ of $T_{W ; \bar{Y}}$ represented by $\mathcal{X}$, since y is fully faithful. Hence $\widetilde{\mathcal{X}}$ is a sheaf.

We denote by $y:$ Top $\rightarrow \mathcal{T}$ the Yoneda embedding. In order to simplify the notation in the following proof, we also denote by $y: B_{T o p} G \rightarrow B_{G}$ the induced functor, for any topological group $G$. By [4, Corollary 3 , the full sub-category of $B_{W_{k(v)}}$ defined by the family of objects

$$
\left\{y\left(W_{k(v)} \times Z\right) ; Z \in O b(T o p)\right\}
$$


is a generating sub-category, for any valuation $v$. Here $y\left(W_{k(v)}\right)$ acts on

$$
y\left(W_{k(v)} \times Z\right)=y\left(W_{k(v)}\right) \times y(Z)
$$

on the first factor. Consider the sequence adjoint functors between $B_{W_{K v}}$ and $B_{W_{K}}$,

$$
\theta_{v !} ; \theta_{v}^{*} ; \theta_{v *},
$$

induced by the morphism of topological groups $\theta_{v}: W_{K_{v}} \rightarrow W_{K}$. Recall that the functor $\theta_{v !}$ is defined by

$$
\theta_{v !}(\mathcal{F})=y\left(W_{K}\right) \times{ }^{y\left(W_{K_{v}}\right)} \mathcal{F}:=\left(y\left(W_{K}\right) \times \mathcal{F}\right) / y\left(W_{K_{v}}\right),
$$

where $y\left(W_{K_{v}}\right)$ acts on the left on $\mathcal{F}$ and by right-translations on $y\left(W_{K}\right)$. For any $v \in \bar{Y}^{0}$ and any $Z \in O b(T o p)$, we define

$$
\mathcal{G}_{Z ; v}=\left(\left(\theta_{v !}\left(q_{v}^{*}\left(y\left(W_{k(v)} \times Z\right)\right)\right) ; y\left(W_{k(v)} \times Z\right) ;\left(\emptyset_{B_{W_{k(w)}}}\right)_{w \neq v_{0} ; v}\right) ; \quad\left(g_{Z ; v}\right)\right),
$$

where the morphism

$$
g_{Z ; v}: q_{v}^{*}\left(y\left(W_{k(v)} \times Z\right)\right) \longrightarrow \theta_{v}^{*} \circ \theta_{v !}\left(q_{v}^{*}\left(y\left(W_{k(v)} \times Z\right)\right)\right)
$$

is given by adjunction. For the trivial valuation $v_{0}$ and for any $Z \in O b(T o p)$, we define

$$
\mathcal{G}_{Z ; v_{0}}:=\left(y\left(W_{K} \times Z\right) ;\left(\emptyset_{B_{W_{k(v)}}}\right)_{v \neq v_{0}}\right) .
$$

Note that $T_{W ; \bar{Y}}$ is equivalent to a full sub-category of $\mathfrak{F}_{W ; \bar{Y}}$, by Proposition 5.4.

Proposition 5.8. The category $T_{W ; \bar{Y}}$ is a generating sub-category of $\mathfrak{F}_{W ; \bar{Y}}$.

Proof. It is shown in the proof of Proposition 3.3 that the family

$$
\left\{\mathcal{G}_{Z ; v} ; Z \in O b(T o p) ; v \in Y\right\}
$$

is a generating family of $\mathfrak{F}_{W ; \bar{Y}}$. Hence it remains to show that $\mathcal{G}_{Z ; v}$ lies in $T_{W ; \bar{Y}}$, for any $Z \in O b(T o p)$ and any $v \in \bar{Y}$. It is obvious for the trivial valuation $v=v_{0}$. Take a non-trivial valuation $v \neq v_{0}$. One has

$$
\theta_{v !}\left(q_{v}^{*}\left(y\left(W_{k(v)} \times Z\right)\right)\right):=y\left(W_{K}\right) \times{ }^{y\left(W_{K_{v}}\right)} y\left(W_{k(v)} \times Z\right)=y\left(W_{K} \times{ }^{W_{K_{v}}}\left(W_{k(v)} \times Z\right)\right),
$$

as it is shown in the proof of (44, Lemma 13). Note that $W_{K_{v}}$ acts on the right on $W_{K}$ and by left translation on the first factor on $\left(W_{k(v)} \times Z\right)$. This defines the quotient space $\left(W_{K} \times W_{K_{v}}\left(W_{k(v)} \times Z\right)\right)$. Then $W_{K}$ acts on $\left(W_{K} \times W_{K_{v}}\left(W_{k(v)} \times Z\right)\right)$ by left translations on the first factor. We obtain

$$
\begin{aligned}
\mathcal{G}_{Z ; v} & =\left(\theta_{v !}\left(q_{v}^{*}\left(y\left(W_{k(v)} \times Z\right)\right)\right) ; y\left(W_{k(v)} \times Z\right) ; \quad\left(\emptyset_{B_{W_{k(w)}}}\right)_{w \neq v_{0} ; v} ; g_{Z ; v}\right) \\
& =\mathrm{y}\left(W_{K} \times{ }^{W_{K_{v}}}\left(W_{k(v)} \times Z\right) ; \quad\left(W_{k(v)} \times Z\right) ; \quad\left(\emptyset_{T o p}\right)_{w \neq v_{0} ; v} ; \widetilde{g_{Z ; v}}\right),
\end{aligned}
$$

where

$$
\widetilde{g_{Z ; v}}:\left(W_{k(v)} \times Z\right) \longrightarrow W_{K} \times{ }^{W_{K_{v}}}\left(W_{k(v)} \times Z\right)
$$

is the unique map of $W_{K_{v}}$-topological spaces such that $y\left(\widetilde{g_{Z ; v}}\right)=g_{Z ; v}$ (recall that the Yoneda embedding $y: B_{T o p} W_{K_{v}} \rightarrow B_{W_{K}}$ is fully faithful). Hence $\mathcal{G}_{Z ; v}$ is an object of $T_{W ; \bar{Y}}$ for any $Z \in O b(T o p)$ and any $v \in \bar{Y}$. 
Theorem 5.9. The canonical morphism

$$
\mathfrak{F}_{W ; \bar{Y}} \longrightarrow\left(\widetilde{T_{W ; \bar{Y}} ; \mathcal{J}_{l s}}\right)
$$

is an equivalence of topoi, where $\left(\widetilde{T_{W ; \bar{Y}} ; \mathcal{J}_{l s}}\right)$ is the category of sheaves on the local section site. More generally, the canonical map

$$
\mathfrak{F}_{L / K, S} \longrightarrow\left(\widetilde{T_{L / K, S} ;} \mathcal{J}_{l s}\right)
$$

is an equivalence.

Proof. The functor y $: T_{W ; \bar{Y}} \rightarrow \mathfrak{F}_{W ; \bar{Y}}$ is fully faithful, the topology $\mathcal{J}_{l s}$ is induced by the canonical topology of $\mathfrak{F}_{W ; \bar{Y}}$ and $T_{W ; \bar{Y}}$ is a generating sub-category of $\mathfrak{F}_{W ; \bar{Y}}$. The first claim of the theorem follows from [5], IV.1.2.1. More generally, the proofs of (5.4), (5.5) and (5.8) remain valid for $\mathfrak{F}_{L / K, S}$, where $L / K$ and $S$ are both finite, by replacing $W_{K}$ and $W_{K_{v}}$ with $W_{L / K, S}$ and $\widetilde{W}_{K_{v}}$ respectively.

Corollary 5.10. The canonical map

$$
\lim _{L / K, S} H^{n}\left(T_{L / K, S}, \mathbb{Z}\right) \rightarrow H^{n}\left(T_{W, \bar{Y}}, \mathbb{Z}\right)
$$

is not an isomorphism for $n=2,3$.

Proof. The cohomology of the site $T_{L / K, S}$ (respectively $T_{W ; \bar{Y}}$ ) is by definition the cohomology of the topos $\left(\widetilde{T_{L / K, S} ; \mathcal{J}_{l s}}\right)$ (respectively $\left(\widetilde{T_{W ; \bar{Y}} ; \mathcal{J}_{l s}}\right)$ ), which is in turn equivalent to $\mathfrak{F}_{L / K, S}$ (respectively $\mathfrak{F}_{W: \bar{Y}}$ ) by Theorem [5.9. Therefore this corollary is just a reformulation of Theorem 4.11

Remark 5.11. This corollary points out that Lichtenbaum's Weil-étale cohomology is not defined as the cohomology of a site (i.e. of a topos).

\section{The Artin-Verdier Étale topos}

The Artin-Verdier étale site associated to a number field takes the (ramification at the) archimedean primes into account (see [9] and 2]). This refinement of the étale site is necessary if one wants to naturally obtain the vanishing of the cohomology in degrees greater than three. Recall that $\bar{Y}$ is the set of valuations of a number field $K$.

6.1. The Artin-Verdier étale site of $\bar{Y}$. Here, all schemes are separated and of finite type over $\operatorname{Spec}(\mathbb{Z})$. A connected $\bar{Y}$-scheme is a pair $\bar{X}=\left(X ; X_{\infty}\right)$, where $X$ is a connected $Y$-scheme in the usual sense. When $X$ is empty, $X_{\infty}$ has to be (empty or) a single point over $Y_{\infty}$. If $X$ is not empty, $X_{\infty}$ is a connected open subset of $X(\mathbb{C}) / \sim$. Here, $X(\mathbb{C}) / \sim$ is the quotient of the set of complex valued points of $X$ under the equivalence relation defined by complex conjugation, endowed with the quotient topology. A $\bar{Y}$-scheme is a finite sum of connected $\bar{Y}$-schemes.

A connected étale $\bar{Y}$-scheme is a connected $\bar{Y}$-scheme $\left(X ; X_{\infty}\right)$, where $X / Y$ is étale of finite presentation and $X_{\infty} / Y_{\infty}$ is unramified in the sense that if $y \in Y_{\infty}$ is real, so is any point $x$ of $X_{\infty}$ lying over $y$. An étale $\bar{Y}$-scheme $\bar{X}$ is a finite sum of connected étale $\bar{Y}$-schemes, called the connected components of $\bar{X}$. A morphism $\bar{\phi}:\left(U ; U_{\infty}\right) \rightarrow\left(V ; V_{\infty}\right)$ of étale $\bar{Y}$-schemes is given by a morphism $\phi: U \rightarrow V$ of étale $Y$-schemes which induces a map $\phi_{\infty}: U_{\infty} \rightarrow V_{\infty}$ over $Y_{\infty}$. Fiber products $\bar{U} \times_{\bar{X}} \bar{V}:=\left(U \times_{X} V ; U_{\infty} \times_{X_{\infty}} V_{\infty}\right)$ exist in the category $E t_{\bar{Y}}$ of étale $\bar{Y}$-schemes. 
Definition 6.1. The Artin-Verdier étale site of $\bar{Y}$ is defined by the category $E t_{\bar{Y}}$ of étale $\bar{Y}$-schemes endowed with the topology $\mathcal{J}_{\text {et }}$ generated by the pre-topology for which the coverings are the surjective families.

6.2. The specialization maps. Let $v$ be a valuation of $K$ corresponding to a point of $Y$. We denote by $k(v)$ and $\overline{k(v)}$ the residue field at $v$ and its algebraic closure. The henselization and the strict henselization of $\bar{Y}$ at $v$ are defined as the projective limits

$$
\operatorname{Spec}\left(\mathcal{O}_{\bar{Y} ; v}^{h}\right):=\underbrace{}_{\lim } \bar{U} \text { and } \operatorname{Spec}\left(\mathcal{O}_{\bar{Y} ; v}^{s h}\right):=l_{\lim } \bar{U},
$$

where $\bar{U}$ runs over the filtered system of étale neighborhoods of $v$ in $\bar{Y}$ and the filtered system of étale neighborhoods of $\bar{v}$ in $\bar{Y}$ respectively. Here an étale neighborhood of $v$ in $\bar{Y}$ (respectively of $\bar{v}$ in $\bar{Y}$ ) is given by an étale $\bar{Y}$-scheme $\bar{U}$ endowed with a morphism $(\operatorname{Spec}(k(v)) ; \emptyset) \rightarrow \bar{U}$ over $\bar{Y}$ (respectively endowed with a morphism $(\operatorname{Spec}(\overline{k(v)}) ; \emptyset) \rightarrow \bar{U}$ over $\bar{Y})$. Then, for $v$ ultrametric, the local ring $\mathcal{O}_{\bar{Y} ; v}^{h}:=\mathcal{O}_{\bar{K}}^{D_{v}}$ is henselian with fraction field $K_{v}^{h}$ and with residue field $k(v)$. Respectively, the local $\operatorname{ring} \mathcal{O}_{\bar{Y} ; v}^{s h}:=\mathcal{O}_{\bar{K}}^{I_{v}}$ is strictly henselian with fraction field $K_{v}^{s h}$ and with residue field $\overline{k(v)}$. For an archimedean valuation $v$, one has

$$
\left(\operatorname{Spec}\left(K_{v}^{s h}\right) ; v\right)=\left(\operatorname{Spec}\left(K_{v}^{h}\right) ; v\right)=\underline{l i m} \bar{U},
$$

where $\bar{U}$ runs over the filtered system of $\bar{Y}$-morphisms $(\emptyset ; v) \rightarrow \bar{U}$. The choice of the valuation $\bar{v}$ of $\bar{K}$ lying over $v$ induces an embedding

$$
K_{v}^{s h}=\bar{K}^{I_{v}} \longrightarrow \bar{K} .
$$

For any ultrametric valuation $v$, we get a specialization map over $\bar{Y}$ :

$$
\operatorname{Spec}(\bar{K})=(\operatorname{Spec}(\bar{K}) ; \emptyset) \longrightarrow\left(\operatorname{Spec}\left(\mathcal{O}_{\bar{Y} ; v}^{s h}\right) ; \emptyset\right)=: \bar{Y}_{v}^{s h} .
$$

Such a specialization map over $\bar{Y}$ is also defined for an archimedean valuation $v$ :

$$
\operatorname{Spec}(\bar{K})=(\operatorname{Spec}(\bar{K}) ; \emptyset) \longrightarrow\left(\operatorname{Spec}\left(K_{v}^{s h}\right) ; v\right)=: \bar{Y}_{v}^{s h} .
$$

In what follows, $\bar{Y}_{v}^{\text {sh }}$ denotes the $\bar{Y}$-schemes $\left(\operatorname{Spec}\left(\mathcal{O}_{\bar{Y} ; v}\right) ; \emptyset\right),\left(\operatorname{Spec}\left(K_{v}^{s h}\right) ; v\right)$ and $\operatorname{Spec}(\bar{K})=(\operatorname{Spec}(\bar{K}) ; \emptyset)$ for $v$ ultrametric, archimedean and the trivial valuation respectively.

6.3. The étale topos of $\bar{Y}$. The Artin-Verdier étale topos $\bar{Y}_{e t}$ associated to the Arakelov compactification of $\operatorname{Spec}\left(\mathcal{O}_{K}\right)$ is the category of sheaves of sets on the site $\left(E t_{\bar{Y}} ; \mathcal{J}_{e t}\right)$. We denote by $Y_{e t}$ the usual étale topos of the scheme $Y$.

Proposition 6.2. There is an open embedding

$$
\varphi: Y_{\text {et }} \longrightarrow \bar{Y}_{\text {et }}
$$

corresponding to the open inclusion $Y:=(Y ; \emptyset) \rightarrow \bar{Y}$. For any closed point $v$ of $\bar{Y}$, there is a closed embedding (see [5], IV, Proposition 9.3.4)

$$
u_{v}: B_{G_{k(v)}}^{s m} \longrightarrow \bar{Y}_{e t} .
$$

The closed complement of $Y_{e t}$ in $\bar{Y}_{\text {et }}$ is the image of the closed embedding

$$
u:=\left(u_{v}\right)_{v \in Y_{\infty}}: \coprod_{v \in Y_{\infty}} \underline{S e t} \longrightarrow \bar{Y}_{e t} .
$$


Proof. The map $Y:=(Y ; \emptyset) \rightarrow \bar{Y}$ is a monomorphism in $E t_{\bar{Y}}$, hence the Yoneda embedding defines a sub-object $\varepsilon(Y)$ of the final object of $\bar{Y}_{e t}$. Thus the localization morphism

$$
\bar{Y}_{e t / \varepsilon(Y)} \longrightarrow \bar{Y}_{e t}
$$

is an open embedding. The category $\left(E t_{\bar{Y}}\right)_{/ Y}$ is isomorphic to the usual category $E t_{Y}$ of étale $Y$-schemes. Under this identification, the usual étale topology $\mathcal{J}_{\text {et }}$ on $E t_{Y}$ is the topology $\mathcal{J}_{\text {ind }}$ induced by the forgetful functor

$$
\left(E t_{\bar{Y}}\right)_{/ Y} \rightarrow E t_{\bar{Y}}
$$

where $E t_{\bar{Y}}$ is endowed with the Artin-Verdier étale topology. Moreover, one has an equivalence (see [5], III.5.4)

$$
\left.\widetilde{\left(E t_{Y} ; \mathcal{J}_{e t}\right.}\right) \simeq\left(\left(E t_{\bar{Y}}\right)_{/ Y} ; \mathcal{J}_{\text {ind }}\right) \simeq \bar{Y}_{\text {et } / \varepsilon(Y)} .
$$

The first claim of the proposition follows from (17) and (18).

Assume that $v$ corresponds to an ultrametric valuation and denote by $v \rightarrow \bar{Y}$ the morphism $(\operatorname{Spec}(k(v)) ; \emptyset) \rightarrow \bar{Y}$. The functor

$$
\begin{aligned}
& u_{v}^{*}: \quad E t_{\bar{Y}} \quad \longrightarrow \quad E t_{k(v)} \simeq T_{G_{k(v)}}^{f}, \\
& (\bar{X} \rightarrow \bar{Y}) \longmapsto\left(\bar{X} \times_{\bar{Y}} v \rightarrow \operatorname{Spec}(k(v))\right)
\end{aligned}
$$

is a morphism of left exact sites, where $T_{G_{k(v)}}^{f}$ denotes the category of finite $G_{k(v)^{-}}$ sets endowed with the canonical topology. We denote by

$$
u_{v}: B_{G_{k(v)}}^{s m} \longrightarrow \bar{Y}_{e t}
$$

the induced morphism of topoi. The adjunction transformation $u_{v}^{*} \circ u_{v *} \rightarrow I d$ is an isomorphism (i.e. $u_{v}$ is an embedding).

Now assume that $v$ is an archimedean valuation and denote by $v \rightarrow \bar{Y}$ the morphism $(\emptyset ; v) \rightarrow \bar{Y}$. Again, the functor

$$
\begin{aligned}
& u_{v}^{*}: \quad E t_{\bar{Y}} \quad \longrightarrow \quad \underline{S e t}^{f}=T_{G_{k(v)}}^{f}, \\
& \bar{X} \rightarrow \bar{Y} \longmapsto \bar{X} \times_{\bar{Y}} v \rightarrow(\emptyset ; v)
\end{aligned}
$$

is a morphism of left exact sites, where $\underline{S e t}^{f}$ is the category of finite sets, endowed with the canonical topology. We get an embedding of topoi

$$
u_{v}: \underline{\text { Set }} \longrightarrow \bar{Y}_{\text {et }} \text {. }
$$

For any $v \in \bar{Y}^{0}$, let $(\bar{Y}-v) \rightarrow \bar{Y}$ be the open complement of the closed point $v$. Again, $\varepsilon(\bar{Y}-v)$ is a sub-object of the final object of $\bar{Y}_{e t}$ which yields an open embedding $j: \bar{Y}_{e t / \varepsilon(\bar{Y}-v)} \longrightarrow \bar{Y}_{e t}$. The strictly full sub-category of $\bar{Y}_{e t}$ defined by the objects $X$ such that $j^{*}(X)$ is the final object of $\bar{Y}_{e t / \varepsilon\left(\bar{Y}_{v}\right)}$ is exactly the essential image of $u_{v *}$. In other words, the image of $u_{v}$ is the closed complement of $j$. Hence, $u_{v}$ is a closed embedding. The last claim of the proposition follows from [5], IV.9.4.6.

Corollary 6.3. The family of functors

$$
\left\{u_{v}^{*}: \bar{Y}_{e t} \rightarrow B_{G_{k(v)}}^{s m} ; v \in \bar{Y}^{0}\right\}
$$

is conservative. 
Proof. By [5], VIII.3.13, the family of functors

$$
\left\{u_{v}^{*}: Y_{e t} \rightarrow B_{G_{k(v)}}^{s m} ; v \in Y^{0}\right\}
$$

is conservative. By [5], IV 9.4.1.c, the result follows from Proposition 6.2 .

Let $\mathcal{F}$ be an object of $Y_{\text {et }}$ and let $\mathcal{F}_{v_{0}} \in O b\left(B_{G_{K}}^{s m}\right)$ be its generic stalk. For any archimedean valuation $v$ one has

$$
u_{v}^{*} \circ \varphi_{*}(\mathcal{F}) \simeq \mathcal{F}_{v_{0}}^{I_{v}} .
$$

Let $u: \coprod_{v \in Y_{\infty}} \underline{S e t} \rightarrow \bar{Y}_{e t}$ be the morphism given by the family $\left(u_{v}\right)_{v \in Y_{\infty}}$. Consider the functor

$$
\begin{aligned}
\rho:=u^{*} \varphi_{*}: Y_{\text {et }} & \longrightarrow \coprod_{v \in Y_{\infty}} \text { Set, } \\
\mathcal{F} & \longmapsto\left(\mathcal{F}_{v_{0}}^{I_{v}}\right)_{v \in Y_{\infty}} .
\end{aligned}
$$

Let us consider the category $\left(\amalg_{v \in Y_{\infty}} \underline{\text { Set }}, Y_{e t}, \rho\right)$ defined in [5], IV.9.5.1.

Corollary 6.4. The category $\bar{Y}_{\text {et }}$ is equivalent to $\left(\coprod_{v \in Y_{\infty}} \underline{\text { Set }}, Y_{\text {et }}, \rho\right)$.

Proof. There is a functor

$$
\begin{aligned}
& \Phi: \bar{Y}_{e t} \longrightarrow\left(\coprod_{v \in Y_{\infty}} \underline{\text { Set }}, Y_{\text {et }}, \rho\right), \\
& \mathcal{F} \longmapsto \quad\left(\varphi^{*} \mathcal{F}, u^{*} \mathcal{F}, f\right) \text {, }
\end{aligned}
$$

where $f: u^{*} \mathcal{F} \rightarrow u^{*} \varphi_{*} \varphi^{*} \mathcal{F}$ is given by adjunction. By [5], IV.9.5.4.a and Proposition 6.2. the functor $\Phi$ is an equivalence of categories.

In particular, we have the usual sequences of adjoint functors

$$
\varphi_{!}, \varphi^{*}, \varphi_{*} \text { and } u^{*}, u_{*}, u^{!}
$$

between the categories of abelian sheaves on $\bar{Y}_{e t}, Y_{e t}$ and $Y_{\infty}$ respectively. It follows that $u_{*}$ is exact and $\varphi^{*}$ preserves injective objects since $\varphi_{\text {! }}$ is exact. For any abelian sheaf $\mathcal{A}$ on $\bar{Y}_{\text {et }}$, one has the exact sequence

$$
0 \rightarrow \varphi ! \varphi^{*} \mathcal{A} \rightarrow \mathcal{A} \rightarrow u_{*} u^{*} \mathcal{A} \rightarrow 0,
$$

where the morphisms are given by adjunction. If $\mathcal{A}$ is a sheaf on $Y_{e t}$, the étale cohomology with compact support is defined by $H_{c}^{n}\left(Y_{e t}, \mathcal{A}\right):=H^{n}\left(\bar{Y}_{e t}, \varphi_{!} \mathcal{A}\right)$. To compute the étale cohomology with compact support, we use (20) and observe that the cohomology of the sheaf $u_{*} u^{*} \mathcal{A}$ is trivial in degrees $n \geq 1$ since $u_{*}$ is exact. For example, one has

$$
H_{c}^{n}\left(Y_{\text {et }}, \mathbb{Z}\right)=0,\left(\prod_{Y_{\infty}} \mathbb{Z}\right) / \mathbb{Z} \text { for } n=0,1 \text { and } H_{c}^{n}\left(Y_{\text {et }}, \mathbb{Z}\right)=H^{n}\left(\bar{Y}_{\text {et }}, \mathbb{Z}\right) \text { for } n \geq 2 .
$$

Now consider the constant étale sheaf associated to the discrete abelian group $\mathbb{R}$. By Proposition 6.5 below, one has

$$
H_{c}^{1}\left(Y_{e t}, \mathbb{R}\right)=\left(\prod_{Y_{\infty}} \mathbb{R}\right) / \mathbb{R} \text { and } H_{c}^{n}\left(Y_{e t}, \mathbb{R}\right)=0 \text { for } n \neq 1 .
$$


6.4. Artin-Verdier étale cohomology. Here we compute the Artin-Verdier étale cohomology with $\mathbb{Z}$-coefficients. Let $j: \operatorname{Spec}(K) \rightarrow Y \rightarrow \bar{Y}$ be the inclusion of the generic point of $\bar{Y}$.

Proposition 6.5. For any uniquely divisible $G_{K}$-module $Q$, the sheaf $j_{*} Q$ on $\bar{Y}_{e t}$ is acyclic for the global sections functor, i.e. $H^{q}\left(\bar{Y}_{e t}, j_{*} Q\right)=0$ for any $q \geq 1$. More generally, if $Q$ is a $G_{K}$-module such that $H^{n}\left(G_{K}, Q\right)=H^{n}\left(I_{v}, Q\right)=0$ for any $n \geq 1$ and any valuation $v$ of $K$, then the sheaf $j_{*} Q$ on $\bar{Y}_{\text {et }}$ is acyclic for the global sections functor.

Proof. Assume that $Q$ is uniquely divisible. The more general case follows from the same argument. For any $v \in \bar{Y}^{0}$, one has (see (19))

$$
u_{v}^{*} R^{q}\left(j_{*}\right) Q=R^{q}\left(u_{v}^{*} j_{*}\right) Q=H^{q}\left(I_{v} ; Q\right) .
$$

The groups $I_{v}$ are all profinite (or finite) and $Q$ is uniquely divisible. We obtain

$$
u_{v}^{*} R^{q}\left(j_{*}\right) Q=0
$$

for any $q \geq 1$. By Corollary 6.3 we get $R^{q}\left(j_{*}\right)(Q)=0$ for any $q \geq 1$. Then the Leray spectral sequence

$$
H^{p}\left(\bar{Y}_{e t} ; R^{q}\left(j_{*}\right) Q\right) \Longrightarrow H^{p+q}\left(G_{K} ; Q\right)
$$

yields

$$
H^{n}\left(\bar{Y}_{e t} ; j_{*} Q\right) \simeq H^{n}\left(G_{K} ; Q\right)=0
$$

for any $n \geq 1$, since Galois cohomology is torsion.

Let $\operatorname{Pic}(Y)$ and $U_{K}$ be the class-group and the unit group of $K$ respectively. Let $r_{1}$ be the number of real primes of $K$. We denote by $A^{D}=H o m(A ; \mathbb{Q} / \mathbb{Z})$ the dual of a finitely generated abelian group (or a profinite group) $A$. We consider the idèle class group $C_{K}$ of $K$ and the connected component $D_{K}$ of $1 \in C_{K}$. The cohomology of the global Galois group $G_{K}$ with coefficients in $\mathbb{Z}$ is trivial in odd degrees, and we have $H^{q}\left(G_{K} ; \mathbb{Z}\right)=\mathbb{Z},\left(C_{K} / D_{K}\right)^{D},(\mathbb{Z} / 2 \mathbb{Z})^{r_{1}}$ for $r=0, r=2$ and $r \geq 4$ even, respectively (see [7], I, Corollary 4.6).

Proposition 6.6. The cohomology of the Artin-Verdier étale topos with coefficients in $\mathbb{Z}$ is given by

$$
\begin{aligned}
H^{q}\left(\bar{Y}_{e t} ; \mathbb{Z}\right) & =\mathbb{Z} \text { for } q=0 \\
& =0 \text { for } q=1 \\
& =\operatorname{Pic}(Y)^{D} \text { for } q=2 \\
& =U_{K}^{D} \text { for } q=3 \\
& =0 \text { for } q \geq 4 .
\end{aligned}
$$

Proof. As in the proof of Proposition 6.5, we get

$$
u_{v}^{*}\left(R^{q}\left(j_{*}\right) \mathcal{L}\right)=R^{q}\left(u_{v}^{*} j_{*}\right) \mathcal{L}=H^{q}\left(I_{v} ; \mathcal{L}\right) \in O b\left(B_{G_{k(v)}}^{s m}\right)
$$

for any $\mathcal{L} \in O b\left(B_{G_{K}}^{s m}\right)$ and any $v \in \bar{Y}$ (recall that $I_{v_{0}}$ is trivial). In particular, one has $j_{*} \mathbb{Z}=\mathbb{Z}$ and

$$
j^{*} R^{q}\left(j_{*}\right)=R^{q}\left(j^{*} j_{*}\right)=R^{q}(I d)=0
$$

for any $q \geq 1$, since $j$ is an embedding. Moreover, the map

$$
R^{q}\left(j_{*}\right) \mathcal{L} \rightarrow \prod_{v \in \bar{Y}^{0}} u_{v *} u_{v}^{*} R^{q}\left(j_{*}\right) \mathcal{L}
$$


given by adjunction factors through $\sum_{v \in \bar{Y}^{0}} u_{v *} H^{q}\left(I_{v} ; \mathcal{L}\right)$, since a cohomology class in $H^{q}\left(G_{K}, \mathcal{L}\right)$ is unramified almost everywhere. The induced map

$$
R^{q}\left(j_{*}\right) \mathcal{L} \longrightarrow \sum_{v \in \bar{Y}^{0}} u_{v *} H^{q}\left(I_{v} ; \mathcal{L}\right)
$$

is an isomorphism using Corollary 6.3 and the fact that $u_{v}^{*}$ commutes with sums. We obtain $R^{q}\left(j_{*}\right) \mathbb{Z}=0$ for $q$ odd. By local class field theory, we have

$$
R^{2}\left(j_{*}\right) \mathbb{Z}=\sum_{v \in Y^{0}} u_{v *}\left(\mathcal{O}_{K_{v}}^{\times}\right)^{D} \sum_{v \in K(\mathbb{R})} u_{v *}(\mathbb{Z} / 2 \mathbb{Z})^{D}
$$

and $R^{q}\left(j_{*}\right) \mathbb{Z}=\sum_{v \in K(\mathbb{R})} u_{v *}(\mathbb{Z} / 2 \mathbb{Z})^{D}$ for $q \geq 4$ even. The Leray spectral sequence

$$
H^{p}\left(\bar{Y}_{e t}, R^{q}\left(j_{*}\right) \mathbb{Z}\right) \Longrightarrow H^{p+q}\left(G_{K}, \mathbb{Z}\right)
$$

yields the exact sequence

$$
\begin{aligned}
0 \rightarrow H^{2}\left(\bar{Y}_{e t}, \mathbb{Z}\right) \rightarrow\left(C_{K} / D_{K}\right)^{D} & \rightarrow \sum_{v \nmid \infty}\left(\mathcal{O}_{K_{v}}^{\times}\right)^{D} \sum_{v \in K(\mathbb{R})}(\mathbb{Z} / 2 \mathbb{Z})^{D} \\
& \rightarrow H^{3}\left(\bar{Y}_{e t}, \mathbb{Z}\right) \rightarrow H^{3}\left(G_{K}, \mathbb{Z}\right)=0,
\end{aligned}
$$

where the central map is the Pontryagin dual of the canonical morphism

$$
\prod_{v \nmid \infty}\left(\mathcal{O}_{K_{v}}^{\times}\right) \prod_{v \in K(\mathbb{R})} \mathbb{Z} / 2 \mathbb{Z} \longrightarrow\left(C_{K} / D_{K}\right) .
$$

The result follows for $q \leq 3$. Next the Leray spectral sequence yields

$$
0 \rightarrow H^{q}\left(\bar{Y}_{e t}, \mathbb{Z}\right) \rightarrow H^{q}\left(G_{K}, \mathbb{Z}\right) \rightarrow \sum_{v \in K(\mathbb{R})} \mathbb{Z} / 2 \mathbb{Z} \rightarrow H^{q+1}\left(\bar{Y}_{e t}, \mathbb{Z}\right) \rightarrow H^{q+1}\left(G_{K}, \mathbb{Z}\right)=0
$$

for any even $q \geq 4$. This ends the proof since the map $H^{q}\left(G_{K}, \mathbb{Z}\right) \rightarrow \sum_{v \in K(\mathbb{R})} \mathbb{Z} / 2 \mathbb{Z}$ is an isomorphism.

The cohomology groups $H^{n}\left(\bar{Y}_{e t}, \mathbb{Z}\right)$ for $n=0,1,2$ can also be deduced from unramified class field theory (i.e. $\left.\pi_{1}\left(\bar{Y}_{e t}\right)^{a b} \simeq C l(K)\right)$ using Proposition 6.5.

\section{THE MORPHISM FROM THE FLASK TOPOS TO THE ÉTALE TOPOS}

In this section we describe the relation between the flask topos and the étale topos. There is a morphism of topoi from the full flask topos $\mathfrak{F}_{W ; \bar{Y}}$ to $\bar{Y}_{e t}$. However, this morphism does not factor through $\mathfrak{F}_{L / K, S}$, and we have to decompose the étale topos as a projective limit in order to understand the relation between the projective system of topoi $\mathfrak{F}_{\bullet}$ and $\bar{Y}_{e t}$.

7.1. The morphism from the étale site to the local section site. By Corollary 5.7, the local section topology $\mathcal{J}_{l s}$ on the category $T_{W ; \bar{Y}}$ is sub-canonical. Since $T_{W ; \bar{Y}}$ has finite projective limits, $\left(T_{W ; \bar{Y}} ; \mathcal{J}_{l s}\right)$ is what we call a left exact site.

Proposition 7.1. There exists a morphism of left exact sites

$$
\begin{aligned}
& \zeta^{*}:\left(E t_{\bar{Y}} ; \mathcal{J}_{\text {et }}\right) \longrightarrow\left(T_{W ; \bar{Y}} ; ; \mathcal{J}_{l s}\right), \\
& \bar{X} \quad \longmapsto \quad \mathcal{X} .
\end{aligned}
$$


Proof. Let $\bar{X}$ be an étale $\bar{Y}$-scheme. For any valuation $v$, we define

$$
X_{v}:=\operatorname{Hom}_{\bar{Y}}\left(\bar{Y}_{v}^{s h} ; \bar{X}\right) \text {. }
$$

Note that, for any ultrametric valuation $v$, the set

$$
X_{v}=\operatorname{Hom}_{\bar{Y}}\left(\bar{Y}_{v}^{s h} ; \bar{X}\right)=\operatorname{Hom}_{\bar{Y}}(\operatorname{Spec}(\overline{k(v)}) ; \bar{X})
$$

carries an action of $G_{k(v)}$. For any archimedean valuation $v$,

$$
X_{v}=\operatorname{Hom}_{\bar{Y}}\left(\bar{Y}_{v}^{s h} ; \bar{X}\right)=\operatorname{Hom}_{\bar{Y}}((\emptyset ; \bar{v}) ; \bar{X})
$$

is just a set. For the trivial valuation $v=v_{0}$,

$$
X_{v_{0}}=\operatorname{Hom}_{\bar{Y}}\left(\bar{Y}_{v_{0}}^{s h} ; \bar{X}\right)=\operatorname{Hom}_{\bar{Y}}(\operatorname{Spec}(\bar{K}) ; \bar{X})
$$

is a $G_{K}$-set. For any valuation $v, X_{v}$ is viewed as a $W_{k(v)}$-topological space. The morphisms (15) and (16) yield maps of $W_{K_{v}}$-spaces $f_{v}: X_{v} \rightarrow X_{v_{0}}$, for any $v$. So we get an object $\zeta^{*}(\bar{X})=\mathcal{X}$ of $T_{W ; \bar{Y}}$. Clearly, $\zeta^{*}$ is a functor. It preserves final objects and fiber products by the universal property of fiber products in the category $E t_{\bar{Y}}$. Hence $\zeta^{*}$ is left exact. Furthermore, an étale cover $\left\{\bar{X}_{i} \rightarrow \bar{X} ; i \in I\right\}$ yields a surjective family of finite $G_{k(v)}$-sets $\left\{X_{i, v} \rightarrow X_{v} ; i \in I\right\}$ for any valuation $v$, hence a local section cover. It follows that $\zeta^{*}$ is continuous and left exact.

This morphism of left exact sites induces a morphism of topoi. Hence the next result follows from Theorem 5.9 .

Corollary 7.2. There is a morphism of topoi $\zeta: \mathfrak{F}_{W: \bar{Y}} \rightarrow \bar{Y}_{e t}$.

The next proposition is an application of (Grothendieck) Galois Theory. This result will not be used in the remaining part of this paper. A proof can be found in 8 .

Proposition 7.3. The functor $\zeta^{*}$ is fully faithful. The essential image of $\zeta^{*}$ is defined by the objects $\mathcal{X}$ of $T_{W ; \bar{Y}}$ such that $X_{v_{0}}$ is a finite set, $f_{v}$ is injective for any $v$ and bijective for almost all $v$ (i.e. except for a finite number of non-trivial valuations). Finally, the étale topology $\mathcal{J}_{\text {et }}$ on $E t_{\bar{Y}}$ is induced via $\zeta^{*}$ by the local section topology $\mathcal{J}_{l s}$ on $T_{W ; \bar{Y}}$.

Remark 7.4. Proposition 7.3 suggests that we define the "Weil-étale topology" as the full sub-category of $T_{W ; \bar{Y}}$ consisting of objects $\left(X_{v}, f_{v}\right)$ such that $f_{v}$ is a topological immersion for every valuation $v$ and a homeomorphism for almost all valuations. Then we endow this full sub-category of $T_{W ; \bar{Y}}$ with the topology induced by the local section topology via the inclusion functor.

7.2. Direct definition of the morphism $\zeta$. For any valuation $v$ of $K$, the specialization map $\bar{Y}_{v}^{s h} \rightarrow \bar{Y}$ induces the co-specialization map

$$
f_{v}: \mathcal{F}_{\bar{v}} \longrightarrow \mathcal{F}_{\bar{v}_{0}}
$$

for any étale sheaf $\mathcal{F}$ on $\bar{Y}$. Here $\mathcal{F}_{\bar{v}}$ and $\mathcal{F}_{\bar{v}_{0}}$ denote the stalks of the sheaf $\mathcal{F}$ at the geometric points $\bar{v} \rightarrow \bar{Y}$ and $\bar{v}_{0} \rightarrow \bar{Y}$. The map (23) is $G_{K_{v}}$-equivariant and functorial in $\mathcal{F}$. More precisely, denote by $\mathfrak{q}_{v}: G_{K_{v}} \rightarrow G_{k(v)}$ the canonical projection and by $\mathfrak{o}_{v}: G_{K_{v}} \rightarrow G_{K}$ the morphism induced by the choice of the valuation $\bar{v}$ of $\bar{K}$ lying over $v$. One has $u_{v}^{*}(\mathcal{F}) \in O b\left(B_{G_{k(v)}}^{s m}\right)$ and $u_{v_{0}}^{*}(\mathcal{F}) \in O b\left(B_{G_{K}}^{s m}\right)$. Then

$$
f_{v}: \mathfrak{q}_{v}^{*}\left(u_{v}^{*} \mathcal{F}\right) \longrightarrow \mathfrak{o}_{v}^{*}\left(u_{v_{0}}^{*} \mathcal{F}\right)
$$


is a map of $B_{G_{K_{v}}}^{s m}$, where we denote a morphism of topological groups and the induced morphism of classifying topoi by the same symbol. Since the squares
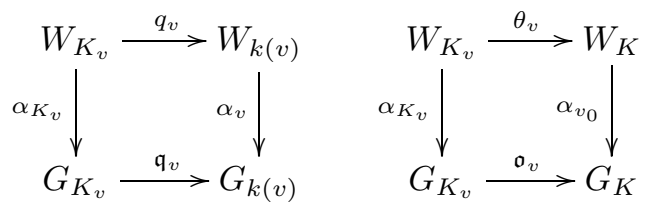

are both commutative, we get a morphism of $B_{W_{K_{v}}}$ :

$$
\alpha_{K_{v}}^{*} f_{v}: q_{v}^{*}\left(\alpha_{v}^{*} \circ u_{v}^{*} \mathcal{F}\right)=\alpha_{K_{v}}^{*} \circ \mathfrak{q}_{v}^{*}\left(u_{v}^{*} \mathcal{F}\right) \longrightarrow \alpha_{K_{v}}^{*} \circ \mathfrak{o}_{v}^{*}\left(u_{v_{0}}^{*} \mathcal{F}\right)=\theta_{v}^{*}\left(\alpha_{v_{0}}^{*} \circ u_{v_{0}}^{*} \mathcal{F}\right) .
$$

We obtain an object

$$
\zeta^{*}(\mathcal{F}):=\left(\alpha_{v}^{*} \circ u_{v}^{*} \mathcal{F} ; \alpha_{K_{v}}^{*} f_{v}\right)_{v \in \bar{Y}}
$$

of the category $\mathfrak{F}_{W ; \bar{Y}}$. This yields a functor

$$
\begin{array}{rlc}
\zeta^{*}: \bar{Y}_{e t} & \longrightarrow & \mathfrak{F}_{W ; \bar{Y}}, \\
\mathcal{F} & \longmapsto\left(\alpha_{v}^{*} \circ u_{v}^{*} \mathcal{F} ; \alpha_{K_{v}}^{*} f_{v}\right)_{v \in \bar{Y}} .
\end{array}
$$

Here the equivariant map of $G_{K_{v}}$-sets

$$
f_{v}: \mathfrak{q}_{v}^{*}\left(u_{v}^{*} \mathcal{F}\right) \longrightarrow \mathfrak{o}_{v}^{*}\left(u_{v_{0}}^{*} \mathcal{F}\right)
$$

is induced by the usual co-specialization map between the stalks of the étale sheaf $\mathcal{F}$.

Proposition 7.5. The functor $\zeta^{*}: \bar{Y}_{e t} \longrightarrow \mathfrak{F}_{W: \bar{Y}}$ is the inverse image of a morphism of topoi

$$
\zeta: \mathfrak{F}_{W ; \bar{Y}} \longrightarrow \bar{Y}_{\text {et }} .
$$

Proof. Since the functors $\alpha_{v}^{*} \circ u_{v}^{*}$ and $\alpha_{K_{v}}^{*}$ commute with finite projective limits and arbitrary inductive limits, so does the functor $\zeta^{*}$, by Proposition 3.2. Since the functor $\zeta^{*}$ is left exact and has a right adjoint, it is the pull-back of a morphism of topoi $\zeta: \mathfrak{F}_{W ; \bar{Y}} \longrightarrow \bar{Y}_{e t}$.

7.3. Equivalence of the two definitions. Here we denote by

$$
z:\left(E t_{\bar{Y}} ; \mathcal{J}_{e t}\right) \longrightarrow\left(T_{W ; \bar{Y}} ; ; \mathcal{J}_{l s}\right)
$$

the morphism of left exact sites defined in Proposition 7.1, We have a commutative square

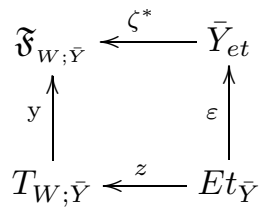

where $\zeta^{*}$ is defined in Proposition 7.5, $y$ is defined in Proposition 5.4 and $\varepsilon: E t_{\bar{Y}} \rightarrow$ $\bar{Y}_{e t}$ is the Yoneda embedding. By [5], IV, Proposition 4.9.4, the morphism of topoi induced by the morphism of left exact sites $z$ is isomorphic to the morphism of topoi $\zeta: \mathfrak{F}_{W: \bar{Y}} \rightarrow \bar{Y}_{e t}$ of Proposition 7.5 .

Proposition 7.6. The morphism $\zeta: \mathfrak{F}_{W ; \bar{Y}} \rightarrow \bar{Y}_{\text {et }}$ is not connected (i.e. the inverse image functor $\zeta^{*}$ is not fully faithful). 
The second definition of the morphism $\zeta: \mathfrak{F}_{W ; \bar{Y}} \rightarrow \bar{Y}_{\text {et }}$ yields a description of its inverse image functor $\zeta^{*}$. This can be used to prove the proposition above (see [8], Corollary 4.67).

7.4. The morphisms $\zeta_{L, S}$. Let $L / K$ be a finite Galois sub-extension of $\bar{K} / K$. We denote by $E t_{L / K}$ the full sub-category of $E t_{\bar{Y}}$ consisting of étale $\bar{Y}$-schemes $\bar{X}$ such that the action of $G_{K}$ on the finite set

$$
X_{v_{0}}=\operatorname{Hom}_{\bar{Y}}(\operatorname{Spec}(\bar{K}) ; \bar{X})
$$

factors through $G_{L / K}=\operatorname{Gal}(L / K)$. This category is endowed with the topology (again denoted by $\mathcal{J}_{\text {et }}$ ) induced by the étale topology on $E t_{\bar{Y}}$ via the inclusion functor $E t_{L / K} \rightarrow E t_{\bar{Y}}$. This functor yields a morphism of left exact sites

$$
\left(E t_{L / K}, \mathcal{J}_{\text {et }}\right) \longrightarrow\left(E t_{\bar{Y}}, \mathcal{J}_{\text {et }}\right)
$$

and a morphism of topoi. These morphisms are compatible, hence they induce a morphism from $\bar{Y}_{\text {et }}$ to the projective limit topos $\underbrace{}_{\lim }\left(E t_{L / K}, \mathcal{J}_{\text {et }}\right)$, where the limit is taken over all the finite Galois sub-extensions of $\bar{K} / K$.

Proposition 7.7. The canonical morphism

$$
\bar{Y}_{e t} \longrightarrow \underset{\lim }{\longleftarrow}\left(\widetilde{E t_{L / K},} \mathcal{J}_{e t}\right)
$$

is an equivalence.

Proof. The morphism of left exact sites

$$
\left(E t_{L / K}, \mathcal{J}_{\text {et }}\right) \longrightarrow\left(E t_{L^{\prime} / K}, \mathcal{J}_{\text {et }}\right)
$$

is given by the inclusion functor, for $\bar{K} / L^{\prime} / L / K$. By [5], VI, 8.2.3, the direct limit site

$$
\underset{\lim }{\longrightarrow}\left(E t_{L / K}, \mathcal{J}_{e t}\right):=\left(\lim _{\longrightarrow}\left(E t_{L / K}\right), \mathcal{J}\right)
$$

is a site for the inverse limit topos $\underline{\lim }\left(\widetilde{E t_{L / K}, \mathcal{J}_{\text {et }}}\right)$. The direct limit category $\underset{\lim }{\longrightarrow}\left(E t_{L / K}\right)$ (see [1], III.3) is canonically equivalent to $E t_{\bar{Y}}$. The topology $\mathcal{J}$ is the coarsest topology which makes all the functors

$$
\left(E t_{L / K}, \mathcal{J}_{\text {et }}\right) \longrightarrow\left(E t_{\bar{Y}}, \mathcal{J}\right)
$$

continuous. In other words, $\mathcal{J}$ is the coarsest topology on $E t_{\bar{Y}}$ such that any covering family of $\left(E t_{L / K}, \mathcal{J}_{\text {et }}\right)$ is a covering family of $E t_{\bar{Y}}$, for all $L / K$. Hence $\mathcal{J}$ is just the étale topology, and $\left(E t_{\bar{Y}}, \mathcal{J}_{e t}\right)$ is a site for the inverse limit topos.

Proposition 7.8. There is a morphism of topoi $\zeta_{L, S}: \mathfrak{F}_{L / K, S} \rightarrow\left(\widetilde{\left.E t_{L / K}, \mathcal{J}_{e t}\right)}\right.$. Moreover, the diagram

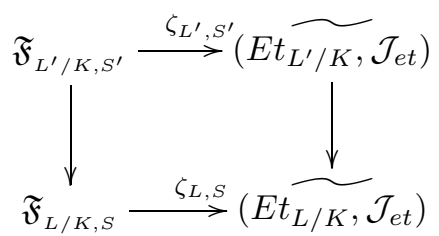

is commutative for $L^{\prime} / L / K$ and $S \subset S^{\prime}$. 
Proof. The functor

$$
\zeta_{L, S}^{*}: E t_{L / K} \longrightarrow T_{L / K, S}
$$

induced by $\zeta^{*}: E t_{\bar{Y}} \longrightarrow T_{W, \bar{Y}}$ yields a morphism of left exact sites; hence the first claim of the proposition follows from Theorem 5.9. The diagram of the proposition is commutative since the corresponding diagram of sites is commutative.

Proposition 7.9. Let $\bar{V}$ be a connected étale $\bar{Y}$-scheme lying in the category $E t_{L / K}$ (i.e. $G_{L / K}$ acts transitively on $V_{v_{0}}$ ). One has an equivalence

$$
\mathfrak{F}_{L / K, S} / \bar{V} \simeq \mathfrak{F}_{L / K(V), \tilde{S}} / \bar{V}
$$

where $K(V)$ is the function field of $\bar{V}$ and $\widetilde{S}$ is the set of places of $K(V)$ lying over $S$.

Proof. The choice of a point of $V_{v_{0}}$ defines an isomorphism of $W_{L / K, S}$-sets

$$
V_{v_{0}} \simeq G_{L} / G_{K(V)}=W_{L / K, S} / W_{L / K(V), \widetilde{S}} .
$$

We get an isomorphism

$$
B_{W_{L / K, S}} / y\left(W_{L / K, S}, V_{v_{0}}\right) \simeq B_{W_{L / K(V), \tilde{s}}}
$$

The same result is valid for any closed point of $\bar{V}$, and the proposition follows.

\section{The SPeCtral Sequence Relating Weil-Étale Cohomology TO ÉTALE COHOMOLOGY}

\subsection{Strongly compact topoi.}

Definition 8.1. A topos $T$ is said to be strongly compact if the functors $H^{n}(T,-)$ commute with filtered colimits of abelian sheaves.

Let $\left(T_{i}, f_{j i}\right)_{i \in I}$ be a filtered projective system of topoi, where the maps $f_{j i}: T_{j} \rightarrow$ $T_{i}$ are the transition maps. We denote by $T_{\infty}:=\lim T_{i}$ the limit topos computed in the 2-category of topoi. We have canonical morphisms $f_{i}: T_{\infty} \rightarrow T_{i}$. Suppose we are given an abelian object $A_{i}$ of $T_{i}$ for any $i \in I$ and a family of morphisms $\alpha_{i j}: f_{j i}^{*} A_{i} \rightarrow A_{j}$ such that the following condition holds:

$$
\alpha_{i k}=\alpha_{j k} \circ f_{k j}^{*}\left(\alpha_{i j}\right): f_{k i}^{*} A_{i}=f_{k j}^{*} f_{j i}^{*} A_{i} \longrightarrow f_{k j}^{*} A_{j} \longrightarrow A_{k} .
$$

In what follows, the data $\left(A_{i}, \alpha_{i j}\right)$ is said to be a compatible system of abelian sheaves on the projective system of topoi $\left(T_{i}, f_{j i}\right)_{i \in I}$.

The morphisms $f_{j}^{*}\left(\alpha_{i j}\right)$ yield a filtered inductive system of abelian objects $\left(f_{i}^{*} A_{i}\right)_{i \in I}$ in $T_{\infty}$, and we set

$$
A_{\infty}:=\underset{\lim }{\longrightarrow} f_{i}^{*} A_{i} .
$$

Lemma 8.2. If the topos $T_{i}$ are all strongly compact, then the canonical morphism

$$
\stackrel{\lim }{\longrightarrow} H^{n}\left(T_{i}, A_{i}\right) \longrightarrow H^{n}\left(T_{\infty}, A_{\infty}\right)
$$

is an isomorphism for any integer $n$.

Proof. By [5], VI, Corollaire 8.7.7 the topos $T_{\infty}$ is strongly compact as well, and one has

$$
H^{n}\left(T_{\infty}, A_{\infty}\right)=\underline{\lim } H^{n}\left(T_{\infty}, f_{i}^{*} A_{i}\right)=\underline{\lim }_{i \in I}\left(\underline{\lim }_{j \rightarrow i} H^{n}\left(T_{j}, f_{j i}^{*} A_{i}\right)\right) .
$$

We easily check that the canonical map

$$
\stackrel{\lim }{\longrightarrow}_{i \in I}\left(\stackrel{\lim }{\longrightarrow} \rightarrow i H^{n}\left(T_{j}, f_{j i}^{*} A_{i}\right)\right) \longrightarrow \underline{\lim }_{i \in I} H^{n}\left(T_{i}, A_{i}\right)
$$


is an isomorphism. The result then follows from the fact that the natural map

$$
\lim _{i \in I}\left(\underline{\lim }_{j \rightarrow i} H^{n}\left(T_{j}, f_{j i}^{*} A_{i}\right)\right) \longrightarrow H^{n}\left(T_{\infty}, A_{\infty}\right)
$$

factors through ${\underset{\lim }{\longrightarrow} i \in I} H^{n}\left(T_{i}, A_{i}\right)$.

Now consider the more general case where the sheaves $A_{i}$ are replaced by bounded below complexes of abelian sheaves $C_{i}^{*}$. Denote by $H^{q}\left(T_{i}, C_{i}^{*}\right)$ the hypercohomology of the complex of sheaves $C_{i}^{*}$. Suppose we are given a compatible family of morphisms of (bounded below) complexes $\alpha_{i j}: f_{j i}^{*} C_{i}^{*} \rightarrow C_{j}^{*}$ for each transition map $f_{j i}: T_{j} \rightarrow T_{i}$. We define

$$
C_{\infty}^{*}:=\lim _{\longrightarrow} f_{i}^{*} C_{i}^{*} .
$$

Lemma 8.3. If the topos $T_{i}$ are all strongly compact, then the canonical morphism

$$
\stackrel{\lim }{\longrightarrow} H^{n}\left(T_{i}, C_{i}^{*}\right) \longrightarrow H^{n}\left(T_{\infty}, C_{\infty}^{*}\right)
$$

is an isomorphism for any integer $n$.

Proof. We denote by $H^{q}\left(C_{i}^{*}\right)$ (respectively $H^{q}\left(C_{\infty}^{*}\right)$ ) the cohomology sheaf of the complex $C_{i}^{*}$ (respectively $C_{\infty}^{*}$ ) in degree $q$. The inverse image functor $f_{i}^{*}$ is exact, hence we have $H^{q}\left(f_{i}^{*} C_{i}^{*}\right)=f_{i}^{*} H^{q}\left(C_{i}^{*}\right)$. By exactness of filtered inductive limits, we obtain

$$
H^{q}\left(C_{\infty}^{*}\right)=\underline{\lim } H^{q}\left(f_{i}^{*} C_{i}\right)=\underline{\lim } f_{i}^{*} H^{q}\left(C_{i}\right)
$$

for any $q \geq 0$. For any $i \in I$, we have a convergent spectral sequence

$$
H^{p}\left(T_{i}, H^{q}\left(C_{i}^{*}\right)\right) \Longrightarrow H^{p+q}\left(T_{i}, C_{i}^{*}\right) .
$$

The compatible morphisms of complexes $\alpha_{i j}: f_{j i}^{*} C_{i}^{*} \rightarrow C_{j}^{*}$ induce compatible morphisms of spectral sequences, hence we have an inductive system of spectral sequences. We obtain a morphism of spectral sequences from

$$
\underset{\lim }{\longrightarrow} H^{p}\left(T_{i}, H^{q}\left(C_{i}^{*}\right)\right) \Longrightarrow \underset{\lim }{\longrightarrow} H^{p+q}\left(T_{i}, C_{i}^{*}\right)
$$

to

$$
H^{p}\left(T_{\infty}, H^{q}\left(C_{\infty}^{*}\right)\right) \Longrightarrow H^{p+q}\left(T_{\infty}, C_{\infty}^{*}\right) .
$$

By the previous lemma, this morphism is an isomorphism at the $E_{2}$-term. It therefore induces isomorphisms on the abutments. The result follows.

Let $\bar{Y}$ be the set of valuations of the number field $K$, and let $\left(C_{L}^{*}, \alpha_{u}\right)$ be a compatible system of bounded below complexes of abelian sheaves on the sites $\left(E t_{L / K}, \mathcal{J}_{e t}\right)_{L}$ (i.e. a bounded below complex of abelian objects in the total topos $\left.\operatorname{Top}\left(E t_{L / K}, \mathcal{J}_{e t}\right)_{L}\right)$. We denote by $C_{\infty}^{*}$ the complex of sheaves on

$$
\bar{Y}_{e t} \simeq \underset{\lim }{\longleftarrow}\left(\widetilde{t_{L / K},} \mathcal{J}_{e t}\right)
$$

defined as above.

Corollary 8.4. We have an isomorphism

$$
\stackrel{\lim }{\longrightarrow} H^{n}\left(E t_{L / K}, C_{L}^{*}\right) \simeq H^{n}\left(\bar{Y}_{e t}, C_{\infty}^{*}\right)
$$

where $L$ runs over the finite Galois sub-extensions of $\bar{K} / K$.

Proof. The topoi $\left(E t_{L / K}, \mathcal{J}_{\text {et }}\right)$ are all coherent and hence strongly compact (see [5], VI, Cor. 5.2). Thus the result follows from the previous lemma. 


\subsection{The spectral sequence.}

Theorem 8.5. Let $\mathcal{A}=\left(\mathcal{A}_{L, S}, f_{t}\right)$ be an abelian object of Top $\left(\mathfrak{F}_{\bullet}\right)$. There exists a bounded below complex $R \mathcal{A}$ of abelian sheaves on $\bar{Y}_{\text {et }}$ and an isomorphism

$$
H^{*}\left(\bar{Y}_{\text {et }}, R \mathcal{A}\right) \simeq \underline{H}^{*}\left(\mathfrak{F}_{L / K, S}, \mathcal{A}\right),
$$

where the left hand side is the étale hypercohomology of the complex $R \mathcal{A}$. In particular, one has a spectral sequence relating Lichtenbaum's Weil-étale cohomology to étale cohomology

$$
H^{p}\left(\bar{Y}_{e t}, R^{q} \mathcal{A}\right) \Longrightarrow \underline{H}^{p+q}\left(\mathfrak{F}_{L / K, S}, \mathcal{A}\right),
$$

where $R^{q} \mathcal{A}$ is the cohomology sheaf of the complex $R \mathcal{A}$ in degree $q$. The complex $R \mathcal{A}$ is well defined up to quasi-isomorphism and functorial in $\mathcal{A}$.

Proof. Since $\operatorname{Top}\left(\mathfrak{F}_{\bullet}\right)$ is a topos, the abelian category $A b\left(\operatorname{Top}\left(\mathfrak{F}_{\bullet}\right)\right)$ has enough injectives. We choose an injective resolution

$$
0 \rightarrow \mathcal{A} \rightarrow I_{\bullet}^{0} \rightarrow I_{\bullet}^{1} \rightarrow \ldots
$$

of the abelian objet $\mathcal{A}=\left(\mathcal{A}_{L, S}, f_{t}\right)$. By Proposition 4.3 , this resolution provides us with an injective resolution

$$
0 \rightarrow \mathcal{A}_{L, S} \rightarrow I_{L, S}^{0} \rightarrow I_{L, S}^{1} \rightarrow \ldots
$$

of the abelian sheaf $\mathcal{A}_{L, S}$ on $\mathfrak{F}_{L, S}$, for any pair $(L, S)$, and with a morphism of complexes

$$
t^{*} I_{L, S}^{*} \longrightarrow I_{L^{\prime}, S^{\prime}}^{*},
$$

for any transition map $t$. These morphisms of complexes are compatible in the usual way.

For any map $\left(L^{\prime}, S^{\prime}\right) \rightarrow(L, S)$ in $I / K$, the following diagram commutes:

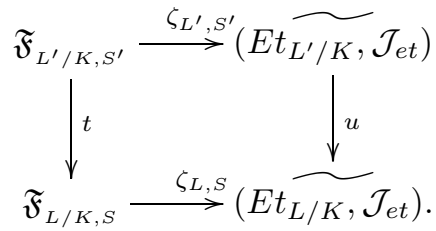

In particular, we have $u_{*} \circ \zeta_{L^{\prime}, S^{\prime}, *} \simeq \zeta_{L, S, *} \circ t_{*}$. By adjunction, we obtain a natural (Beck-Chevalley) transformation

$$
u^{*} \zeta_{L, S, *} \longrightarrow u^{*} \zeta_{L, S, *} t_{*} t^{*} \simeq u^{*} u_{*} \zeta_{L^{\prime}, S^{\prime}, *} t^{*} \longrightarrow \zeta_{L^{\prime}, S^{\prime}, *} t^{*}
$$

This transformation induces a morphism of complexes

$$
u^{*} \zeta_{L, S, *} I_{L, S}^{*} \longrightarrow \zeta_{L^{\prime}, S^{\prime}, *} t^{*} I_{L, S}^{*} \longrightarrow \zeta_{L^{\prime}, S^{\prime}, *} I_{L^{\prime}, S^{\prime}}^{*}
$$

where the last arrow is given by the morphism $t^{*} I_{L, S}^{*} \longrightarrow I_{L^{\prime}, S^{\prime}}^{*}$

For any fixed Galois extension $L / K$, we have in particular a filtered inductive system of complexes of sheaves $\left(\zeta_{L, S, *} I_{L, S}^{*}\right)_{S}$ in the topos $\left(E t_{L / K}, \mathcal{J}_{\text {et }}\right)$. We set

$$
I_{L}^{*}:=\lim _{S} \zeta_{L, S, *} I_{L, S}^{*}
$$

For any transition map $u$, (24) induces a morphism of complexes $u^{*} I_{L}^{*} \rightarrow I_{L^{\prime}}^{*}$, since $u^{*}$ commutes with inductive limits. In other words, $\left(I_{L}^{*}\right)_{L}$ defines a compatible system of complexes of sheaves on the sites $\left(E t_{L / K}\right)_{L}$. 
By definition, the cohomology of the complex $\zeta_{L, S, *} I_{L, S}^{*}$ in degree $n$ is the sheaf $R^{n}\left(\zeta_{L, S, *}\right) \mathcal{A}_{L, S}$ of the topos $\left(E t_{L / K}, \mathcal{J}_{e t}\right)$. By exactness of filtered inductive limits, the cohomology of the complex $I_{L}^{*}:=\lim _{S} \zeta_{L, S, *} I_{L, S}^{*}$ in degree $n$ is the sheaf $\lim _{S} R^{n}\left(\zeta_{L, S, *}\right) \mathcal{A}_{L, S}$. We denote this sheaf by $\mathcal{A}_{L}^{(n)}$. Then we have

$$
H^{n}\left(I_{L}^{*}\right)=\lim _{S} H^{n}\left(\zeta_{L, S, *} I_{L, S}^{*}\right)=\lim _{S} R^{n}\left(\zeta_{L, S, *}\right) \mathcal{A}_{L, S}=: \mathcal{A}_{L}^{(n)} .
$$

Since the inverse image functor $u_{L}^{*}$ of the morphism $u_{L}: \bar{Y}_{\text {et }} \rightarrow\left(\widetilde{E t_{L / K}, \mathcal{J}_{\text {et }}}\right)$ is exact, the cohomology of the complex $u_{L}^{*}\left(I_{L}^{*}\right)$ is given by the sheaves

$$
H^{n}\left(u_{L}^{*}\left(I_{L}^{*}\right)\right)=u_{L}^{*} H^{n}\left(I_{L}^{*}\right)=u_{L}^{*} \mathcal{A}_{L}^{(n)} .
$$

Passing to the limit over $L / K$, we define the complex

$$
R \mathcal{A}:=\lim _{L / K} u_{L}^{*}\left(I_{L}^{*}\right) .
$$

The cohomology sheaf of this complex in degree $n$ is given by

$$
R^{n} \mathcal{A}:=H^{n}(R \mathcal{A})={\underset{\lim }{\longrightarrow}{ }_{L / K}} H^{n}\left(u_{L}^{*} I_{L}^{*}\right)={\underset{\lim }{\longrightarrow / K}}_{L} u_{L}^{*} H^{n}\left(I_{L}^{*}\right)=\underline{\lim }_{L^{\prime K K}} u_{L}^{*} \mathcal{A}_{L}^{(n)} .
$$

Now consider a fixed Galois extension $L / K$. For any $S$, the Leray spectral sequence associated to the composition

$$
\mathfrak{F}_{L / K, S} \longrightarrow\left(E t_{L / K}, \mathcal{J}_{\text {et }}\right) \longrightarrow \underline{\text { Set }}
$$

yields an isomorphism

$$
H^{p}\left(E t_{L / K}, \zeta_{L, S, *} I_{L, S}^{*}\right) \simeq H^{p+q}\left(\mathfrak{F}_{L / K, S}, \mathcal{A}_{L, S}\right),
$$

where the first term is the hypercohomology of the complex $\zeta_{L, S, *} I_{L, S}^{*}$ on the site $E t_{L / K}$.

The complexes $\left(\zeta_{L, S, *} I_{L, S}^{*}\right)_{S}$ form an inductive system whose colimit is $I_{L}^{*}$, when $S$ runs over the finite sets of valuations of $K$ containing the archimedean ones and those which ramify in $L$. Passing to the limit over $S$, we obtain an isomorphism

$$
H^{n}\left(E t_{L / K}, I_{L}^{*}\right) \simeq \lim _{\longrightarrow} H^{n}\left(E t_{L / K}, \zeta_{L, S, *} I_{L, S}^{*}\right) \simeq \lim _{S} H^{n}\left(\mathfrak{F}_{L / K, S}, \mathcal{A}_{L, S}\right)
$$

Here, the first isomorphism follows from Lemma 8.3 (taking $T_{i}$ to be constantly $\left.\left(\widetilde{E t}_{L / K}, \mathcal{J}_{\text {et }}\right)\right)$.

We have shown above that the family of complexes $I_{L}$ forms a compatible system when $L / K$ runs over the set of finite Galois sub-extensions of $\bar{K} / K$. Passing to the limit over $L / K$, we obtain

$$
H^{n}\left(\bar{Y}_{\text {et }}, R \mathcal{A}\right)=H^{n}\left(\bar{Y}_{\text {et }}, \lim _{L} u_{L}^{*}\left(I_{L}^{*}\right)\right) \simeq \lim _{L} H^{n}\left(E t_{L / K}, I_{L}^{*}\right)
$$

by Corollary 8.4. Therefore, isomorphisms (27) and (28) yield

$H^{n}\left(\bar{Y}_{\text {et }}, R \mathcal{A}\right) \simeq \underline{\lim }_{L} H^{n}\left(E t_{L / K}, I_{L}^{*}\right) \simeq \underline{\lim }_{L} \lim _{S} H^{n}\left(\mathfrak{F}_{L / K, S}, \mathcal{A}_{L, S}\right) \simeq \underline{H}^{n}\left(\mathfrak{F}_{L / K, S}, \mathcal{A}\right)$, for any $n \geq 0$.

Let us now show that the complex of étale sheaves $R \mathcal{A}$ is well defined up to quasi-isomorphism. This complex has been defined by an injective resolution $I_{\bullet}$ of $\mathcal{A}$ in $\mathfrak{F}_{\bullet}$. Let $I_{\bullet}^{*}$ and $J_{\bullet}^{*}$ be two injective resolutions of $\mathcal{A}$. Denote by $R \mathcal{A}\left(I_{\bullet}\right)$ and $R \mathcal{A}\left(J_{\bullet}\right)$ the étale complexes defined as above. There is a morphism

$$
q_{\bullet}: I_{\bullet}^{*} \longrightarrow J_{\bullet}^{*}
$$


of complexes of abelian objects in $\mathfrak{F} \bullet$ well defined up to homotopy. For any pair $(L, S)$, we have in particular a morphism

$$
q_{L, S}: I_{L, S}^{*} \longrightarrow J_{L, S}^{*}
$$

over $I d_{\mathcal{A}_{L, S}}$. Applying $\zeta_{L, S, *}$, the morphism $q_{L, S}$ induces a quasi-isomorphism

$$
\zeta_{L, S, *} I_{L, S}^{*} \longrightarrow \zeta_{L, S, *} J_{L, S}^{*},
$$

since

$$
R^{q}\left(\zeta_{L, S, *}\right) \mathcal{A}_{L, S}:=H^{q}\left(\zeta_{L, S, *} I_{L, S}^{*}\right) \simeq H^{q}\left(\zeta_{L, S, *} J_{L, S}^{*}\right) .
$$

If $L / K$ is fixed, the morphisms $q_{L, S}$ induce a morphism of complexes

$$
q_{L}: I_{L}^{*}:=\lim _{S} \zeta_{L, S, *} I_{L, S}^{*} \longrightarrow J_{L}^{*}:=\lim _{S} \zeta_{L, S, *} J_{L, S}^{*}
$$

which is a quasi-isomorphism by (25). Passing to the limit over $L / K$, the morphisms $q_{L}$ induce a morphism

$$
R \mathcal{A}\left(I_{\bullet}\right):=\underline{\lim }_{L / K} u_{L}^{*}\left(I_{L}^{*}\right) \longrightarrow \underline{\lim }_{L / K} u_{L}^{*}\left(J_{L}^{*}\right)=: R \mathcal{A}\left(J_{\bullet}\right) .
$$

This is a quasi-isomorphism by (26), hence $R \mathcal{A}$ is well defined up to a quasi-isomorphism.

Let $\mathcal{A}=\left(\mathcal{A}_{L, S}, f_{t}\right)$ be an abelian object of $\operatorname{Top}\left(\mathfrak{F}_{\bullet}\right)$ and let $\bar{U}$ be an étale $\bar{Y}$ scheme. If $L / K$ is large enough so that the $G_{K}$-action on the finite set $U_{v_{0}}$ factors through $G(L / K)$, then $\bar{U}$ defines an object $\zeta^{*}(\bar{U})$ of $\mathfrak{F}_{L / K, S}$. We consider the cohomology groups

$$
H^{*}\left(\mathfrak{F}_{L / K, S}, \bar{U}, \mathcal{A}_{L, S}\right):=H^{*}\left(\mathfrak{F}_{L / K, S} / \zeta^{*}(\bar{U}), \mathcal{A}_{L, S} \times \zeta^{*}(\bar{U})\right)
$$

of the slice topos $\mathfrak{F}_{L / K, S} / \zeta^{*}(\bar{U})$. For any étale $\bar{Y}$-scheme $\bar{U}$, the direct limit

$$
\underline{H}^{*}\left(\mathfrak{F}_{L / K, S}, \bar{U}, \mathcal{A}\right):=\underline{\lim }_{\longrightarrow, S} H^{*}\left(\mathfrak{F}_{L / K, S}, \bar{U}, \mathcal{A}_{L, S}\right)
$$

is well defined. It follows from Proposition 7.9 that the computation of these cohomology groups can be reduced to the case of an open sub-scheme $\bar{U}$ of $\bar{Y}$, as defined in Definition 4.12. Therefore, one can apply Proposition 4.14 and Proposition 4.15 to obtain explicit computations.

Proposition 8.6. The sheaf $R^{q} \mathcal{A}$ is the sheaf associated to the presheaf

$$
\begin{aligned}
\mathcal{P}^{q} \mathcal{A}: \quad E t_{\bar{Y}} & \longrightarrow \\
\bar{U} & \longmapsto \underline{H}^{q}\left(\mathfrak{F}_{L / K, S}, \underline{A b}, \bar{U}, \mathcal{A}\right) .
\end{aligned}
$$

Proof. The sheaf $R^{q}\left(\zeta_{L, S, *}\right) \mathcal{A}_{L, S}$ is the sheaf associated to the presheaf

$$
\begin{aligned}
\mathcal{P}_{L, S}^{q}: \quad E t_{L / K} & \longrightarrow \quad \underline{A b}, \bar{U} H^{q}\left(\mathfrak{F}_{L / K, S}, \bar{U}, \mathcal{A}_{L, S}\right) .
\end{aligned}
$$

It follows that the sheaf

$$
\mathcal{A}_{L}^{(q)}:=\lim _{S} R^{q}\left(\zeta_{L, S, *}\right) \mathcal{A}_{L, S}
$$

is the sheaf associated to the presheaf

$$
\begin{aligned}
\mathcal{P}_{L}^{q}:=\lim _{S} \mathcal{P}_{L, S}^{q}: \quad E t_{L / K} & \longrightarrow \\
\bar{U} & \longmapsto \lim _{S} H^{q}\left(\mathfrak{F}_{L / K, S}, \underline{A b}, \overline{\left.\mathcal{A}_{L, S}\right) .}\right.
\end{aligned}
$$

Indeed, the associated sheaf functor commutes with inductive limit, since it is the inverse image of a morphism of topoi. The morphism of left exact sites

$$
u_{L}^{*}:\left(E t_{L / K}, \mathcal{J}_{e t}\right) \longrightarrow\left(E t_{\bar{Y}}, \mathcal{J}_{\text {et }}\right)
$$


induces the following commutative diagram of topoi:

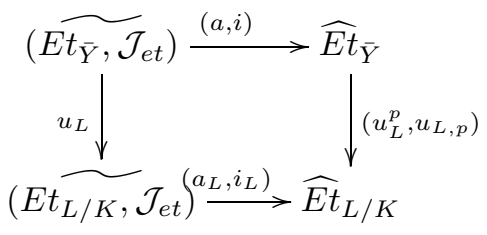

where $\widehat{E t}_{\bar{Y}}$ (respectively $\widehat{E t}_{L / K}$ ) denotes the category of presheaves on $E t_{\bar{Y}}$ (respectively on $\left.E t_{L / K}\right)$. To check the commutativity of this diagram, we observe the direct images of these morphisms, for which the commutativity is obvious. Therefore we have

$$
a \circ u_{L}^{p} \simeq u_{L}^{*} \circ a_{L},
$$

where $a$ and $a_{L}$ are the associated sheaf functors. We obtain

$$
u_{L}^{*} \mathcal{A}_{L}^{(q)}:=u_{L}^{*} \circ a_{L}\left(\mathcal{P}_{L}^{q}\right)=a \circ u_{L}^{p}\left(\mathcal{P}_{L}^{q}\right),
$$

and finally

$$
R^{q} \mathcal{A}:=\underline{\lim }_{L} u_{L}^{*} \mathcal{A}_{L}^{(q)}=\lim _{L} a \circ u_{L}^{p}\left(\mathcal{P}_{L}^{q}\right)=a\left(\underline{\lim }_{L} u_{L}^{p}\left(\mathcal{P}_{L}^{q}\right)\right),
$$

where the last identification comes from the fact that $a$ commutes with inductive limits. In other words, $R^{q} \mathcal{A}$ is the étale sheaf on $\bar{Y}$ associated to the presheaf $\lim _{L} u_{L}^{p}\left(\mathcal{P}_{L}^{q}\right)$. This presheaf can be made explicit as follows. For any connected étale $\bar{Y}$-scheme $\bar{V}$, one has

$$
\left[\underline{\lim }_{L} u_{L}^{p}\left(\mathcal{P}_{L}^{q}\right)\right](\bar{V})=\underline{\lim }_{L}\left[u_{L}^{p}\left(\mathcal{P}_{L}^{q}\right)(\bar{V})\right]=\underline{\lim }_{L}\left[\underline{\lim }_{\bar{V} \rightarrow \bar{U}} \mathcal{P}_{L}^{q}(\bar{V})\right],
$$

where the second limit is taken over the category of arrows $\bar{V} \rightarrow \bar{U}$, for $\bar{U}$ running through the class of objects of $E t_{L / K}$. The first identification can be justified by saying that the limits of presheaves are computed component-wise (i.e. "arguments par arguments").

If $L / K$ is large enough so that $\bar{V}$ is an object of $E t_{L / K}$, then one has

$$
{\underset{\lim }{\longrightarrow} \bar{V} \rightarrow \bar{U}} \mathcal{P}_{L}^{q}(\bar{U})=\mathcal{P}_{L}^{q}(\bar{V})
$$

since $I d_{\bar{V}}$ is then the initial object of the category of arrows $\bar{V} \rightarrow \bar{U}$, for $\bar{U}$ in $E t_{L / K}$. We obtain the following identifications:

$$
\begin{aligned}
{\left[\stackrel{\lim }{\longrightarrow}_{L} u_{L}^{p}\left(\mathcal{P}_{L}^{q}\right)\right](\bar{V}) } & =\stackrel{\lim }{\longrightarrow}_{L}\left[\underline{\lim }_{\bar{V} \rightarrow \bar{U}} \mathcal{P}_{L}^{q}(\bar{U})\right]=\underline{\lim }_{L} \mathcal{P}_{L}^{q}(\bar{V}) \\
& =\stackrel{\lim }{\longrightarrow}_{L} \underline{\lim }_{S} H^{q}\left(\mathfrak{F}_{L / K, S}, \bar{V}, \mathcal{A}_{L / K, S}\right) .
\end{aligned}
$$

Therefore, $R^{q} \mathcal{A}$ is the étale sheaf on $\bar{Y}$ associated to the presheaf

$$
\begin{aligned}
& \mathcal{P}^{q} \mathcal{A}: \quad E t_{\bar{Y}} \longrightarrow \\
& \bar{V} \longmapsto \\
& \lim _{L, S} H^{q}\left(\mathfrak{F}_{L / K, S}, \bar{V}, \mathcal{A}_{L / K, S}\right)=: \underline{H}^{q}\left(\mathfrak{F}_{L / K, S}, \bar{V}, \mathcal{A}\right) .
\end{aligned}
$$

Below we consider the sheaves $\mathbb{Z}$ and $\widetilde{\mathbb{R}}$ of the total topos $T o p\left(\mathfrak{F}_{\bullet}\right)$ defined in Example 4.2 and the sheaves $\phi ! \mathbb{Z}$ and $\phi ! \widetilde{\mathbb{R}}$ defined in section 4.3 Finally, we consider the étale sheaves $\varphi ! \mathbb{Z}$ and $\varphi ! \mathbb{R}$ defined via the open inclusion $\varphi: Y_{e t} \rightarrow \bar{Y}_{e t}$, where here $\mathbb{R}$ denotes the constant sheaf on $Y_{\text {et }}$ associated to the discrete abelian group $\mathbb{R}$. We assume below that $K$ is totally imaginary. 
Corollary 8.7. One has the following results:

(1) $R^{0}(\mathbb{Z})=\mathbb{Z}$ and $R^{q}(\mathbb{Z})=0$ for $q \geq 1$ odd.

(2) $R^{q}(\widetilde{\mathbb{R}})=\mathbb{R}$ for $q=0,1$ and $R^{q}(\widetilde{\mathbb{R}})=0$ for $q \geq 2$.

(3) $R^{0}(\phi ! \mathbb{Z})=\varphi ! \mathbb{Z}$ and $R^{q}(\phi ! \mathbb{Z})=R^{q} \mathbb{Z}$ for $q \geq 1$.

(4) $R^{q}\left(\phi_{!} \widetilde{\mathbb{R}}\right)=\varphi_{!} \mathbb{R}$ for $q=0,1$ and $R^{q}\left(\phi_{!} \widetilde{\mathbb{R}}\right)=0$ for $q \geq 2$.

Proof. Using Proposition 8.6 (and Proposition 7.9), this follows from Propositions 4.14 and 4.15, and equations (8) and (9).

\section{9. ÉtAle COMPLEXes FOR the WeIL-ÉTALE COHOMOLOGY}

In this section, we assume that the number field $K$ is totally imaginary. In order to obtain the relevant Weil-étale cohomology (i.e. the vanshing of the cohomology in degrees $i \geq 4$ ), we need to truncate the complex $R \mathbb{Z}$. However, a non-trivial preliminary condition has to be satisfied. Namely the sheaf $R^{2} \mathbb{Z}$, which fills the gap between Weil-étale and étale cohomology, should be acyclic for the global sections functor on $\bar{Y}$. We study this sheaf below, and we show that it has the right cohomology using an indirect argument. Then we define complexes of étale sheaves computing the conjectural Weil-étale cohomology.

9.1. Cohomology of the sheaf $R^{2} \mathbb{Z}$. By Proposition 4.14 and Proposition 8.6, the étale sheaf $R^{2} \mathbb{Z}$ is the sheaf associated to the presheaf

$$
\begin{array}{ccc}
\mathcal{P}^{2} \mathbb{Z}: \quad E_{\bar{Y}} & \longrightarrow \\
\bar{V} & \longmapsto & \underline{H}^{2}\left(\mathfrak{F}_{L / K, S}, \overline{\bar{V}}, \mathbb{Z}\right)=\left(C_{\bar{V}}^{1}\right)^{\mathcal{D}} .
\end{array}
$$

The compact group $C_{\bar{V}}^{1}$ is the kernel of the map $C_{\bar{V}} \rightarrow \mathbb{R}$, where $C_{\bar{V}}$ is defined in Definition 4.13, Recall that if $\bar{V}$ is connected of function field $K(\bar{V})$, then $C_{\bar{V}}$ is the $S$-idèle class group of $K(\bar{V})$, where $S$ is the set of places of $K(\bar{V})$ not corresponding to a point of $\bar{V}$. Note that such a finite set $S$ does not necessarily contain all the archimedean places. The restriction maps of the presheaf $\mathcal{P}^{2} \mathbb{Z}$ are induced by the canonical maps $C_{\bar{U}} \rightarrow C_{\bar{V}}$ (well defined for any étale map $\bar{U} \rightarrow \bar{V}$ of connected étale $\bar{Y}$-schemes). By unramified class field theory, one has a covariantly functorial exact sequence of compact topological groups

$$
0 \rightarrow D_{\bar{V}}^{1} \rightarrow C_{\bar{V}}^{1} \rightarrow \pi_{1}^{a b}(\bar{V}) \rightarrow 0
$$

where $\pi_{1}^{a b}(\bar{V})$ is the abelian étale fundamental group of $\bar{V}$ and $D_{\bar{V}}^{1}$ is the connected component of 1 in $C_{\bar{V}}^{1}$. Here $\pi_{1}^{a b}(\bar{V})$ is defined as the abelianization of the profinite fundamental group of the Artin-Verdier étale topos $\bar{Y}_{e t} / \bar{V} \simeq \bar{V}_{e t}$. If we denote the function field of $\bar{V}$ by $K(\bar{V})$, then this group is just the Galois group of the maximal abelian extension of $K(\bar{V})$ unramified at every place of $K(\bar{V})$ corresponding to a point of $\bar{V}$.

By Pontryagin duality, we obtain a (contravariantly) functorial exact sequence of discrete abelian groups

$$
0 \rightarrow \pi_{1}^{a b}(\bar{V})^{\mathcal{D}} \rightarrow\left(C_{\bar{V}}^{1}\right)^{\mathcal{D}} \rightarrow\left(D_{\bar{V}}^{1}\right)^{\mathcal{D}} \rightarrow 0
$$

i.e. an exact sequence of abelian étale presheaves on $\bar{Y}$. On the one hand, the sheaf associated to the presheaf

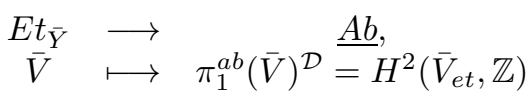


vanishes, and the associated sheaf functor is exact on the other. Therefore, the exact sequence (29) shows that $R^{2} \mathbb{Z}$ is the sheaf associated to the presheaf

$$
\begin{aligned}
& P: E t_{\bar{Y}} \longrightarrow \underline{A b}, \\
& \bar{V} \longmapsto\left(\overline{D_{\bar{V}}^{1}}\right)^{\mathcal{D}} .
\end{aligned}
$$

The connected component $D_{\bar{V}}^{1}$ of the S-idèle class group $C_{\bar{V}}^{1}$ is not known in general, as pointed out to the author by Alexander Schmidt. The computation of the sheaf $R^{2} \mathbb{Z}$ is a delicate problem. We shall compute the cohomology of $R^{2} \mathbb{Z}$ using an indirect argument.

Lemma 9.1. The canonical morphism

$$
\stackrel{H}{H}^{4}\left(\mathfrak{F}_{L / K, S}, \mathbb{Z}\right) \longrightarrow H^{0}\left(\bar{Y}_{\text {et }}, R^{4} \mathbb{Z}\right):=R^{4} \mathbb{Z}(\bar{Y})
$$

is an isomorphism.

Proof. The canonical morphism $\underline{H}^{4}\left(\mathfrak{F}_{L / K, S}, \mathbb{Z}\right) \rightarrow H^{0}\left(\bar{Y}_{e t}, R^{4} \mathbb{Z}\right)$ is induced by the morphism of presheaves $\mathcal{P}^{4} \mathbb{Z} \rightarrow R^{4} \mathbb{Z}$ (see Proposition 8.6). Let

$$
J: B_{W_{K}} \longrightarrow B_{G_{K}}^{s m} \longrightarrow \bar{Y}_{e t}
$$

be the morphism induced by the continuous morphism $W_{K} \rightarrow G_{K}$ and by the inclusion of the generic point of $\bar{Y}$. For any $n \geq 0$, the étale sheaf $R^{n}\left(J_{*}\right) \mathbb{Z}$ is the sheaf associated to the presheaf

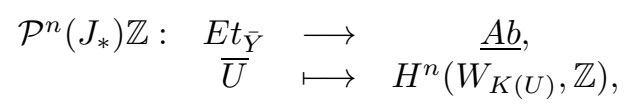

where $\bar{U}$ is assumed to be connected. Here $W_{K(U)}$ is the Weil group of the number field $K(U)$. For any finite extension $K^{\prime} / K$, one has a surjective map (see [4], Proof of Corollary 9)

$$
H^{4}\left(W_{K^{\prime}}, \mathbb{Z}\right) \longrightarrow \sum_{v \in Y_{\infty}^{\prime}} H^{4}\left(W_{K_{v}^{\prime}}, \mathbb{Z}\right)=\sum_{v \in Y_{\infty}^{\prime}} H^{4}\left(\mathbb{S}^{1}, \mathbb{Z}\right)=\sum_{v \in Y_{\infty}^{\prime}} \mathbb{Z}
$$

where $Y_{\infty}^{\prime}$ is the set of archimedean primes of $K^{\prime}$. We denote by

$$
u: \coprod_{Y_{\infty}} \underline{\text { Set }} \longrightarrow \bar{Y}_{e t}
$$

the closed embedding of topoi given by the map $Y_{\infty} \rightarrow \bar{Y}$. Then (30) induces a surjective morphism of presheaves

$$
\mathcal{P}^{4}\left(J_{*}\right) \mathbb{Z} \longrightarrow u_{*} \mathbb{Z} .
$$

We obtain the following exact sequence of presheaves:

$$
0 \longrightarrow \mathcal{P}^{4} \mathbb{Z} \longrightarrow \mathcal{P}^{4}\left(J_{*}\right) \mathbb{Z} \longrightarrow u_{*} \mathbb{Z} \longrightarrow 0,
$$

as it follows from Proposition 8.6 and Proposition 4.14. The associated sheaf functor is exact, hence we have an exact sequence of sheaves

$$
0 \longrightarrow R^{4} \mathbb{Z} \longrightarrow R^{4}\left(J_{*}\right) \mathbb{Z} \longrightarrow u_{*} \mathbb{Z} \longrightarrow 0,
$$

since $u_{*} \mathbb{Z}$ was already a sheaf. We get a long exact sequence

$$
0 \longrightarrow R^{4} \mathbb{Z}(\bar{Y}) \longrightarrow R^{4}\left(J_{*}\right) \mathbb{Z}(\bar{Y}) \longrightarrow \sum_{Y_{\infty}} \mathbb{Z} \longrightarrow \ldots
$$


Moreover, there is a morphism of exact sequences

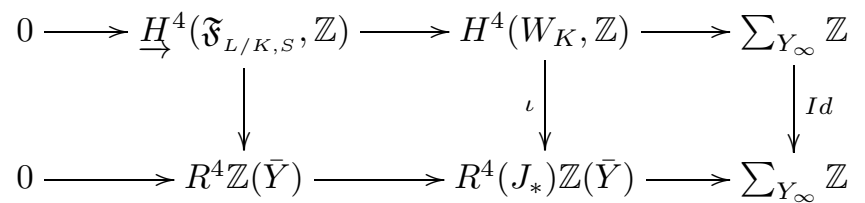

where the vertical maps are the natural ones. Lemma 9.3 shows that $\iota$ is an isomorphism, and the result follows.

We denote by $W_{K}^{1}$ the maximal compact sub-group of the Weil group $W_{K}$. There is a canonical isomorphism of topological groups $W_{K} \simeq W_{K}^{1} \times \mathbb{R}$. We denote by $\mathcal{T}^{l c}$, $B_{W_{K}}^{l c}$ and $B_{W_{K}^{1}}^{l c}$ the topoi obtained by replacing the category of topological spaces $T o p$ with the category $T_{o p}{ }^{l c}$ of locally compact topological spaces with countable basis. In the following two lemmas, we consider the composite morphism

$$
\alpha: B_{W_{K}} \stackrel{h}{\longrightarrow} B_{W_{K}}^{l c} \stackrel{\alpha^{l c}}{\longrightarrow} B_{W_{K}^{1}}^{l c},
$$

where $\alpha^{l c}$ is the morphism of classifying topoi induced by the projection $W_{K} \rightarrow W_{K}^{1}$.

Lemma 9.2. For any $n \geq 1$, one has $R^{n}\left(\alpha_{*}\right) \mathbb{Z}=0$.

Proof. By 4, Prop. 9.1, the direct image $h_{*}$ of the morphism $B_{W_{K}} \stackrel{h}{\longrightarrow} B_{W_{K}}^{l c}$ is exact. The Leray spectral sequence associated to the composite morphism $\alpha$ gives

$$
R^{n}\left(\alpha_{*}\right) \mathbb{Z} \simeq R^{n}\left(\alpha_{*}^{l c}\right) h_{*} \mathbb{Z}=R^{n}\left(\alpha_{*}^{l c}\right) \mathbb{Z} .
$$

It is therefore enough to show that $R^{n}\left(\alpha_{*}^{l c}\right) \mathbb{Z}=0$ for any $n \geq 1$. We consider the pull-back

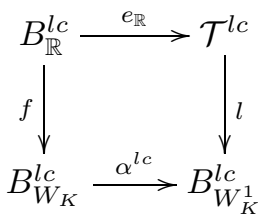

where the vertical arrows are the localization morphisms (one has for example $\left.B_{W_{K}^{1}}^{l c} / E_{W_{K}^{1}} \simeq \mathcal{T}^{l c}\right)$. This pull-back square induces an isomorphism

$$
l^{*} R^{n}\left(\alpha_{*}^{l c}\right) \simeq R^{n}\left(e_{\mathbb{R} *}\right) f^{*} .
$$

One the other hand, the object of $\mathcal{T}^{l c}$

$$
R^{n}\left(e_{\mathbb{R} *}\right) f^{*} \mathbb{Z}=R^{n}\left(e_{\mathbb{R} *}\right) \mathbb{Z}
$$

is represented by the discrete abelian group $H^{n}\left(B_{\mathbb{R}}^{l c}, \mathbb{Z}\right)$ (see [4, Prop. 9.2). This group is trivial for any $n \geq 1$, and we obtain $l^{*} R^{n}\left(\alpha_{*}^{l c}\right) \mathbb{Z}=0$. But $l^{*}$ is faithful, hence $R^{n}\left(\alpha_{*}^{l c}\right) \mathbb{Z}=0$ for any $n \geq 1$. The result follows.

Lemma 9.3. The canonical map

$$
H^{4}\left(W_{K}, \mathbb{Z}\right) \longrightarrow R^{4}\left(J_{*}\right) \mathbb{Z}(\bar{Y})
$$

is an isomorphism. 
Proof. We decompose the morphism $J: B_{W_{K}} \rightarrow \bar{Y}_{e t}$ as follows:

$$
J=\beta \circ \alpha: B_{W_{K}} \longrightarrow B_{W_{K}^{1}}^{l c} \longrightarrow \bar{Y}_{e t}
$$

The Leray spectral sequence associated to this composite map and the previous lemma shows that the natural morphism of étale sheaves

$$
R^{n}\left(\beta_{*}\right) \mathbb{Z}=R^{n}\left(\beta_{*}\right)\left(\alpha_{*} \mathbb{Z}\right) \longrightarrow R^{n}\left(J_{*}\right) \mathbb{Z}
$$

is an isomorphism. It is therefore enough to show that the natural map

$$
H^{4}\left(W_{K}, \mathbb{Z}\right) \simeq H^{4}\left(W_{K}^{1}, \mathbb{Z}\right) \longrightarrow R^{4}\left(\beta_{*}\right) \mathbb{Z}(\bar{Y})
$$

is an isomorphism (where $H^{4}\left(W_{K}, \mathbb{Z}\right) \simeq H^{4}\left(W_{K}^{1}, \mathbb{Z}\right)$ follows from Lemma 9.2). To this aim, we decompose the morphism $\beta$ as follows:

$$
\beta=\mathrm{j} \circ p: B_{W_{K}^{1}}^{l c} \longrightarrow B_{G_{K}}^{s m} \longrightarrow \bar{Y}_{e t} .
$$

This provides us with the Leray spectral sequence

$$
R^{i}\left(\mathrm{j}_{*}\right) \circ R^{j}\left(p_{*}\right) \mathbb{Z} \Longrightarrow R^{i+j}\left(\beta_{*}\right) \mathbb{Z}
$$

We denote by $M^{j}$ the $G_{K}$-module $R^{j}\left(p_{*}\right) \mathbb{Z}$. By [4], equation (21), and 4], Lemma 11 , one has

$$
M^{j}=0 \text { for } j \text { odd. }
$$

For any $G_{K}$-module $M$, the étale sheaf $R^{i}\left(\mathrm{j}_{*}\right) M$ is the sheaf associated to the presheaf

$$
\bar{U} \mapsto H^{i}\left(B_{G_{K}}^{s m}, U_{v_{0}}, M\right)=H^{i}\left(G_{K(U)}, M\right) .
$$

It is well known that a totally imaginary number field is of strict cohomological dimension 2. Hence we have $R^{i}\left(\mathrm{j}_{*}\right) M=0$ for $i \geq 3$. The proof of [4], Lemma 12 (b) shows that $H^{i}\left(G_{K(U)}, M^{2}\right)=0$ for any $i \geq 1$. Hence the group

$$
R^{i}\left(\mathrm{j}_{*}\right) \circ R^{j}\left(p_{*}\right) \mathbb{Z}=R^{i}\left(\mathrm{j}_{*}\right) M^{j}
$$

is trivial for $i \geq 3$, or if the index $j$ is odd, or for $(j=2, i \geq 1)$. The initial term of the spectral sequence

$$
R^{i}\left(\mathrm{j}_{*}\right) M^{j}:=R^{i}\left(\mathrm{j}_{*}\right) \circ R^{j}\left(p_{*}\right) \mathbb{Z} \Longrightarrow R^{i+j}\left(\beta_{*}\right) \mathbb{Z}
$$

therefore looks as follows:

$\begin{array}{cccccc}0 & 0 & 0 & 0 & 0 & 0 \\ \mathrm{j}_{*} M^{4} & R^{1} \mathrm{j}_{*} M^{4} & R^{2} \mathrm{j}_{*} M^{4} & 0 & 0 & 0 \\ 0 & 0 & 0 & 0 & 0 & 0 \\ \mathrm{j}_{*} M^{2} & 0 & 0 & 0 & 0 & 0 \\ 0 & 0 & 0 & 0 & 0 & 0 \\ \mathrm{j}_{*} M^{0} & R^{1} \mathrm{j}_{*} M^{0} & R^{2} \mathrm{j}_{*} M^{0} & 0 & 0 & 0 .\end{array}$

This yields a natural isomorphism

$$
R^{4}\left(\beta_{*}\right) \mathbb{Z} \simeq \mathrm{j}_{*} M^{4},
$$

and we obtain the following identifications:

$$
R^{4}\left(\beta_{*}\right) \mathbb{Z}(\bar{Y}) \simeq \mathrm{j}_{*} M^{4}(\bar{Y})=H^{0}\left(G_{K}, M^{4}\right) \simeq H^{4}\left(W_{K}^{1}, \mathbb{Z}\right)
$$

Indeed, the last isomorphism in (32) is given by the spectral sequence

$$
H^{i}\left(G_{K}, M^{j}\right) \Longrightarrow H^{i+j}\left(W_{K}^{1}, \mathbb{Z}\right)
$$


which is made explicit in [4, Lemma 12. Note that the isomorphisms in (32) are given by the natural maps

$$
H^{4}\left(W_{K}^{1}, \mathbb{Z}\right) \longrightarrow R^{4}\left(\beta_{*}\right) \mathbb{Z}(\bar{Y}) \longrightarrow H^{0}\left(G_{K}, M^{4}\right) .
$$

Hence (31) is an isomorphism, and the result follows.

Recall that $A^{\mathcal{D}}:=\operatorname{Hom}_{c}(A, \mathbb{R} / \mathbb{Z})$ denotes the Pontryagin dual of a locally compact abelian group $A$. If $A$ is a discrete abelian group, we set $A^{D}:=\operatorname{Hom}(A, \mathbb{Q} / \mathbb{Z})$.

Theorem 9.4. The étale sheaf $R^{2} \mathbb{Z}$ is acyclic for the global section functor $\Gamma_{\bar{Y}}$. In other words, one has $H^{n}\left(\bar{Y}_{\text {et }}, R^{2} \mathbb{Z}\right)=0$ for any $n \geq 1$.

Proof. By Corollary 8.7(1), the initial term of the spectral sequence

$$
H^{p}\left(\bar{Y}, R^{q} \mathbb{Z}\right) \Longrightarrow \underline{H}^{p+q}\left(\mathfrak{F}_{L / K, S}, \mathbb{Z}\right)
$$

looks as follows:

$\begin{array}{cccccc}0 & 0 & 0 & 0 & 0 & 0 \\ H^{0}\left(\bar{Y}, R^{4} \mathbb{Z}\right) & H^{1}\left(\bar{Y}, R^{4} \mathbb{Z}\right) & H^{2}\left(\bar{Y}, R^{4} \mathbb{Z}\right) & H^{3}\left(\bar{Y}, R^{4} \mathbb{Z}\right) & 0 & 0 \\ 0 & 0 & 0 & 0 & 0 & 0 \\ H^{0}\left(\bar{Y}, R^{2} \mathbb{Z}\right) & H^{1}\left(\bar{Y}, R^{2} \mathbb{Z}\right) & H^{2}\left(\bar{Y}, R^{2} \mathbb{Z}\right) & H^{3}\left(\bar{Y}, R^{2} \mathbb{Z}\right) & 0 & 0 \\ 0 & 0 & 0 & 0 & 0 & 0 \\ \mathbb{Z} & 0 & \operatorname{Pic}(Y)^{D} & U_{K}^{D} & 0 & 0 .\end{array}$

We obtain the exact sequence

(34)

$$
0 \rightarrow \operatorname{Pic}(Y)^{D} \rightarrow \operatorname{Pic}^{1}(\bar{Y})^{\mathcal{D}} \rightarrow H^{0}\left(\bar{Y}_{e t}, R^{2} \mathbb{Z}\right) \rightarrow U_{K}^{D} \rightarrow \mu_{K}^{D} \rightarrow H^{1}\left(\bar{Y}_{e t}, R^{2} \mathbb{Z}\right) \rightarrow 0 .
$$

The group $H^{1}\left(\bar{Y}_{e t}, R^{2} \mathbb{Z}\right)$ is trivial since the canonical map $U_{K}^{D} \rightarrow \mu_{K}^{D}$ is surjective. Then, this spectral sequence gives the exact sequence

$$
\begin{aligned}
0 & \rightarrow H^{2}\left(\bar{Y}_{e t}, R^{2} \mathbb{Z}\right) \rightarrow \underline{H}^{4}\left(\mathfrak{F}_{L / K, S}, \mathbb{Z}\right) \rightarrow H^{0}\left(\bar{Y}_{e t}, R^{4} \mathbb{Z}\right) \\
\rightarrow H^{3}\left(\bar{Y}_{e t}, R^{2} \mathbb{Z}\right) & \rightarrow \underline{H}^{5}\left(\mathfrak{F}_{L / K, S}, \mathbb{Z}\right)=0,
\end{aligned}
$$

where the central map is an isomorphism by Lemma 9.1 We get

$$
H^{2}\left(\bar{Y}_{e t}, R^{2} \mathbb{Z}\right)=H^{3}\left(\bar{Y}_{e t}, R^{2} \mathbb{Z}\right)=0 .
$$

Finally, the group $H^{n}\left(\bar{Y}_{\text {et }}, R^{2} \mathbb{Z}\right)$ is trivial for any $n \geq 4$ since the étale site of $\bar{Y}$ is of strict cohomological dimension 3 (see [3]).

In order to compute the group $H^{0}\left(\bar{Y}_{e t}, R^{2} \mathbb{Z}\right)$, one needs to study the sheaf $R^{2} \mathbb{Z}$ in more detail. There is a canonical map

$$
\operatorname{Pic}{ }^{1}(\bar{Y})^{\mathcal{D}} \longrightarrow \operatorname{Hom}\left(U_{K}, \mathbb{Z}\right) \longrightarrow \operatorname{Hom}\left(U_{K}, \mathbb{Q}\right) \text {. }
$$

One can show that the morphism

$$
P i c^{1}(\bar{Y})^{\mathcal{D}} \simeq \stackrel{H}{H}^{2}\left(\mathfrak{F}_{L / K, S}, \mathbb{Z}\right) \longrightarrow H^{0}\left(\bar{Y}_{e t}, R^{2} \mathbb{Z}\right)
$$


factors through an injective map

$$
c: \operatorname{Hom}\left(U_{K}, \mathbb{Q}\right) \longrightarrow H^{0}\left(\bar{Y}_{e t}, R^{2} \mathbb{Z}\right) .
$$

Then one can show that this gives a morphism of exact sequences

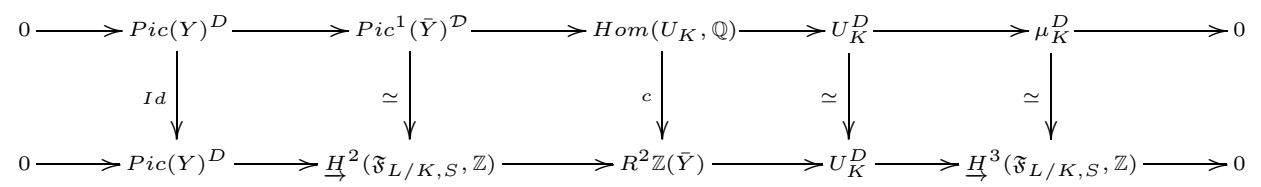

where the bottom row is the exact sequence given by the spectral sequence (33). It follows that (35) is an isomorphism. But this fact will not be used in the remaining part of this paper.

\subsection{The complexes.}

Theorem 9.5. There exists a complex $R_{W} \mathbb{Z}$ of étales sheaves on $\bar{Y}_{\text {et }}$, well defined up to quasi-ismorphism, whose hypercohomology is the expected Weil-étale cohomology:

$$
\begin{aligned}
H^{n}\left(\bar{Y}_{e t} ; R_{W} \mathbb{Z}\right) & =\mathbb{Z} \text { for } n=0 \\
& =0 \text { for } n=1 \\
& =\operatorname{Pic}^{1}(\bar{Y})^{\mathcal{D}} \text { for } n=2 \\
& =\mu_{K}^{D} \text { for } n=3 \\
& =0 \text { for } n \geq 4 .
\end{aligned}
$$

Proof. Consider the complex $R \mathbb{Z}$. The truncated complex

$$
R_{W} \mathbb{Z}=\tau_{\leq 2} R \mathbb{Z}
$$

is also well defined up to quasi-isomorphism. One has $H^{n}\left(\tau_{\leq 2} R \mathbb{Z}\right)=H^{n}(R \mathbb{Z})$ for $n \leq 2$ and $H^{n}\left(\tau_{<2} R \mathbb{Z}\right)=0$ for $n \geq 3$. By Corollary 8.7 and Theorem 9.4 the $E^{2}$-term of the spectral sequence

$$
H^{p}\left(\bar{Y}_{e t}, H^{q}\left(R_{W} \mathbb{Z}\right)\right) \Longrightarrow H^{p+q}\left(\bar{Y}_{e t}, R_{W} \mathbb{Z}\right)
$$

therefore looks like:

$\begin{array}{ccccc}0 & 0 & 0 & 0 & 0 \\ H^{0}\left(\bar{Y}_{e t}, R^{2} \mathbb{Z}\right) & 0 & 0 & 0 & 0 \\ 0 & 0 & 0 & 0 & 0 \\ \mathbb{Z} & 0 & P i c(Y)^{D} & U_{K}^{D} & 0 .\end{array}$

We immediately obtain $H^{0}\left(\bar{Y}_{e t}, R_{W} \mathbb{Z}\right)=\mathbb{Z}$ and $H^{1}\left(\bar{Y}_{e t}, R_{W} \mathbb{Z}\right)=0$. Next the spectral sequence yields the exact sequence

$$
\begin{aligned}
0 & \rightarrow \operatorname{Pic}(Y)^{D} \rightarrow H^{2}\left(\bar{Y}_{e t}, R_{W} \mathbb{Z}\right) \rightarrow H^{0}\left(\bar{Y}_{e t}, R^{2} \mathbb{Z}\right) \\
& \rightarrow \operatorname{Hom}\left(U_{K}, \mathbb{Q} / \mathbb{Z}\right) \rightarrow H^{3}\left(\bar{Y}_{e t}, R_{W} \mathbb{Z}\right) \rightarrow 0 .
\end{aligned}
$$


Note that it is already clear that $R_{W} \mathbb{Z}$ has the expected hypercohomology. The morphism of complexes $R_{W} \mathbb{Z} \rightarrow R \mathbb{Z}$ induces a morphism of spectral sequences from

$$
H^{p}\left(\bar{Y}_{e t}, H^{q}\left(R_{W} \mathbb{Z}\right)\right) \Longrightarrow H^{p+q}\left(\bar{Y}_{e t}, R_{W} \mathbb{Z}\right)
$$

to

$$
H^{p}\left(\bar{Y}_{e t}, H^{q}(R \mathbb{Z})\right) \Longrightarrow \stackrel{H}{\rightarrow}^{p+q}\left(\mathfrak{F}_{L / K, S}, \mathbb{Z}\right)
$$

which in turn induces a morphism of exact sequences from (36) to (34). We obtain

$$
H^{n}\left(\bar{Y}_{e t}, R_{W} \mathbb{Z}\right) \simeq \stackrel{H}{\rightarrow}^{n}\left(\mathfrak{F}_{L / K, S}, \mathbb{Z}\right) \text { for } n=2,3 .
$$

The result for $n=2,3$ then follows from Theorem 4.10. Finally, the groups $H^{n}\left(\bar{Y}_{e t}, R_{W} \mathbb{Z}\right)$ vanish for $n \geq 4$, since the diagonals $\{p+q=n, n \geq 4\}$ of this spectral sequence are trivial.

Theorem 9.6. There exists a complex $R_{W}(\phi ! \mathbb{Z})$ of étales sheaves on $\bar{Y}_{\text {et }}$, well defined up to quasi-ismorphism, whose hypercohomology is the expected Weil-étale cohomology with compact support:

$$
\begin{aligned}
H^{n}\left(\bar{Y}_{e t} ; R_{W}(\phi ! \mathbb{Z})\right) & =0 \text { for } n=0 \\
& =\left(\prod_{Y_{\infty}} \mathbb{Z}\right) / \mathbb{Z} \text { for } n=1 \\
& =\operatorname{Pic}^{1}(\bar{Y})^{\mathcal{D}} \text { for } n=2 \\
& =\mu_{K}^{D} \text { for } n=3 \\
& =0 \text { for } n \geq 4 .
\end{aligned}
$$

Proof. The morphism $\phi ! \mathbb{Z} \rightarrow \mathbb{Z}$ in $T$ op $(\mathfrak{F} \bullet)$ induces a morphism of étale complexes $R \phi ! \mathbb{Z} \rightarrow R \mathbb{Z}$. We obtain a morphism of truncated complexes

$$
R_{W}(\phi ! \mathbb{Z}):=\tau_{\leq 2} R(\phi ! \mathbb{Z}) \longrightarrow R_{W}(\mathbb{Z}):=\tau_{\leq 2} R \mathbb{Z}
$$

inducing a morphism of spectral sequences. Using Corollary 8.7(3), we obtain

$$
H^{n}\left(\bar{Y}_{e t} ; R_{W}(\phi ! \mathbb{Z})\right)=H^{n}\left(\bar{Y}_{e t} ; R_{W}(\mathbb{Z})\right)
$$

for $n \geq 2$. Finally, the spectral sequence

$$
H^{p}\left(\bar{Y}_{e t} ; H^{q}\left(R_{W} \phi ! \mathbb{Z}\right)\right) \Longrightarrow H^{p+q}\left(\bar{Y}_{e t} ; R_{W}(\phi ! \mathbb{Z})\right)
$$

yields $H^{n}\left(\bar{Y}_{e t} ; R_{W}\left(\phi_{!} \mathbb{Z}\right)\right)=H^{n}\left(\bar{Y}_{e t} ; \varphi ! \mathbb{Z}\right)$ for $n=0,1$. The result follows from (21).

Theorem 9.7. The hypercohomology of the complex of étale sheaves $R\left(\phi_{!} \widetilde{\mathbb{R}}\right)$ is given by

$$
\begin{aligned}
H^{n}\left(\bar{Y}_{e t} ; R\left(\phi_{!} \widetilde{\mathbb{R}}\right)\right) & =0 \text { for } n=0 \\
& =\left(\prod_{Y_{\infty}} \mathbb{R}\right) / \mathbb{R} \text { for } n=1,2 \\
& =0 \text { for } n \geq 3 .
\end{aligned}
$$

Proof. The spectral sequence

$$
H^{p}\left(\bar{Y}_{e t} ; R^{q}\left(\phi_{!} \widetilde{\mathbb{R}}\right)\right) \Longrightarrow H^{p+q}\left(\bar{Y}_{e t} ; R\left(\phi_{!} \widetilde{\mathbb{R}}\right)\right)
$$

degenerates and yields

$$
H^{n}\left(\bar{Y}_{e t} ; R(\phi ! \widetilde{\mathbb{R}})\right)=H^{1}\left(\bar{Y}_{e t}, R^{n-1}(\phi ! \widetilde{\mathbb{R}})\right)=H^{1}\left(\bar{Y}_{e t}, \varphi ! \mathbb{R}\right) \text { for } n=1,2
$$


and $H^{n}\left(\bar{Y}_{e t} ; R\left(\phi_{!} \widetilde{\mathbb{R}}\right)\right)=0$ for $n \neq 1,2$ (see Corollary $\left.8.7(4)\right)$. Hence the result follows from (22).

\section{ACKNOWLEDGMENTS}

The author is very grateful to Matthias Flach for his questions, comments and suggestions which led to a more complete and useful article.

\section{REFERENCES}

[1] M. Artin, Grothendieck Topologies. Notes on a Seminar, Harvard University, 1962.

[2] M. Bienenfeld, An étale cohomology duality theorem for number fields with a real embedding. Trans. Amer. Math. Soc. 303 (1987), no. 1, 71-96. MR896008 (88j:12004)

[3] C. Deninger, An extension of Artin-Verdier duality to nontorsion sheaves. J. Reine Angew. Math. 366 (1986), 18-31. MR833011 (87m:11055)

[4] M. Flach, Cohomology of topological groups with applications to the Weil group. Compositio Math. 144 (2008), no. 3, 633-656. MR2422342 (2009f:14033)

[5] A. Grothendieck, M. Artin and J.L. Verdier, Théorie des Topos et cohomologie étale des schémas (SGA4). Lectures Notes in Math. 269, 270, 305, Springer, 1972. MR0354653(50:7131)

[6] S. Lichtenbaum, The Weil-étale topology for Number Rings. To appear in Ann. of Math. MR2552104(2011a:14035)

[7] J.S. Milne, Arithmetic Duality Theorems. Perspectives in Mathematics 1, Academic Press, Inc., Boston, Mass., 1996. MR881804 (88e:14028)

[8] B. Morin, Sur le topos Weil-étale d'un corps de nombres. Thesis, 2008.

[9] T. Zink, Etale cohomology and duality in number fields. Haberland, Galois cohomology, Berlin, 1978, Appendix 2.

Department of Mathematics, California Institute of Technology, 1200 E. California Boulevard, Pasadena, California 91125 